J. Inst. Math. Jussieu (2022), 21(6), 2043-2091

doi:10.1017/S1474748021000104 $\quad$ (C) The Author(s), 2021. Published by Cambridge University 2043 Press. This is an Open Access article, distributed under the terms of the Creative Commons

Attribution licence (http://creativecommons.org/licenses/by/4.0/), which permits unrestricted re-use, distribution, and reproduction in any medium, provided the original work is properly cited.

\title{
SPECIAL VALUES OF THE ZETA FUNCTION OF AN ARITHMETIC SURFACE
}

\author{
MATTHIAS FLACH (D) AND DANIEL SIEBEL \\ Dept. of Mathematics 253-37, California Institute of Technology, Pasadena CA 91125 \\ (flach@caltech.edu, daniel.siebel@yahoo.de)
}

(Received 31 August 2020; revised 4 February 2021; accepted 6 February 2021; first published online 15 March 2021)

\begin{abstract}
We prove that the special-value conjecture for the zeta function of a proper, regular, flat arithmetic surface formulated in [6] at $s=1$ is equivalent to the Birch and Swinnerton-Dyer conjecture for the Jacobian of the generic fibre. There are two key results in the proof. The first is the triviality of the correction factor of [6, Conjecture 5.12], which we show for arbitrary regular proper arithmetic schemes. In the proof we need to develop some results for the eh-topology on schemes over finite fields which might be of independent interest. The second result is a different proof of a formula due to Geisser, relating the cardinalities of the Brauer and the Tate-Shafarevich group, which applies to arbitrary rather than only totally imaginary base fields.
\end{abstract}

Keywords and phrases: arithmetic surfaces, zeta values, Weil-étale cohomology, Birch and SwinnertonDyer conjecture

2020 Mathematics Subject Classification: Primary 14F20

Secondary $14 \mathrm{~F} 42 ; 11 \mathrm{G} 40$

\section{Introduction}

Let $\mathcal{X}$ be a regular scheme of dimension $d$, proper over $\operatorname{Spec}(\mathbb{Z})$. In $[6$, Conjectures 5.11 and 5.12], the first author and Morin formulated a conjecture on the vanishing order and the leading Taylor coefficient of the zeta function $\zeta(\mathcal{X}, s)$ of $\mathcal{X}$ at integer arguments $s=n \in \mathbb{Z}$ in terms of what we call Weil-Arakelov cohomology complexes. More specifically, the conjecture involves a certain invertible $\mathbb{Z}$-module

$$
\Delta(\mathcal{X} / \mathbb{Z}, n):=\operatorname{det}_{\mathbb{Z}} R \Gamma_{W, c}(\mathcal{X}, \mathbb{Z}(n)) \otimes_{\mathbb{Z}} \operatorname{det}_{\mathbb{Z}} R \Gamma\left(\mathcal{X}_{Z a r}, L \Omega_{\mathcal{X} / \mathbb{Z}}^{<n}\right)
$$

which can be attached to the arithmetic scheme $\mathcal{X}$ under various standard assumption (finite generation of étale motivic cohomology in a certain range being the most important). Under these assumptions, there is a natural trivialisation

$$
\lambda_{\infty}: \mathbb{R} \stackrel{\sim}{\longrightarrow} \Delta(\mathcal{X} / \mathbb{Z}, n) \otimes_{\mathbb{Z}} \mathbb{R}
$$

and we conjecture

$$
\lambda_{\infty}\left(\zeta^{*}(\mathcal{X}, n)^{-1} \cdot C(\mathcal{X}, n) \cdot \mathbb{Z}\right)=\Delta(\mathcal{X} / \mathbb{Z}, n),
$$


which determines the leading coefficient $\zeta^{*}(\mathcal{X}, n) \in \mathbb{R}$ up to sign (all identities in this paper involving leading coefficients should be understood up to $\operatorname{sign}$ ). Here $C(\mathcal{X}, n) \in \mathbb{Q}^{\times}$ is a certain inexplicit correction factor, defined as a product over its $p$-primary parts and where the definition of each $p$-primary part involves $p$-adic Hodge theory. It is easy to see that $C(\mathcal{X}, n)=1$ for $n \leq 0$, and we also have $C(\mathcal{X}, n)=1$ for $\mathcal{X}$ of characteristic $p$. In $[7$, Remark 5.2] it was then suggested that in fact $C(\mathcal{X}, n)$ has the simple form

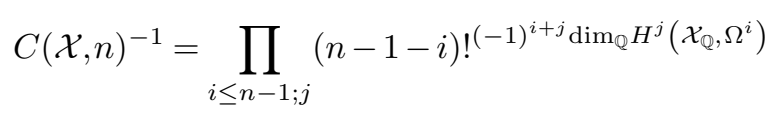

for any $n \in \mathbb{Z}$ and any $\mathcal{X}$. This formula is corroborated by the computation of $C\left(\operatorname{Spec}\left(\mathcal{O}_{F}\right), n\right)$ in $\left[6\right.$, Proposition 5.34] for a number field $F$ whose completions $F_{v}$ are all absolutely abelian.

In the present paper we focus on the case $n=1$ and then specialise further to arithmetic surfaces. We give more evidence for formula (2) by proving that it holds for $n=1$-that is, that $C(\mathcal{X}, 1)=1$ - for arbitrary $\mathcal{X}$. If $\mathcal{X}$ is connected, flat over $\operatorname{Spec}(\mathbb{Z})$ and of dimension $d=2$, we call $\mathcal{X}$ an arithmetic surface. Denoting by $F$ the algebraic closure of $\mathbb{Q}$ in the function field of $\mathcal{X}$, the structural morphism $\mathcal{X} \rightarrow \operatorname{Spec}(\mathbb{Z})$ factors through a morphism

$$
f: \mathcal{X} \rightarrow \operatorname{Spec}\left(\mathcal{O}_{F}\right)=: S
$$

with $f_{*} \mathcal{O}_{\mathcal{X}}=\mathcal{O}_{S}$. We show that all the assumptions entering into our conjecture are satisfied for $n=1$ if $\mathcal{X}$ has finite Brauer group, or equivalently if the Jacobian $J_{F}$ of $\mathcal{X}_{F}$ has finite Tate-Shafarevich group. To give an idea of what the conjecture says concretely, recall that the zeta function of an arithmetic surface factors

$$
\zeta(\mathcal{X}, s)=\frac{\zeta\left(H^{0}, s\right) \zeta\left(H^{2}, s\right)}{\zeta\left(H^{1}, s\right)}=\frac{\zeta_{F}(s) \zeta_{F}(s-1)}{\zeta\left(H^{1}, s\right)},
$$

where $\zeta\left(H^{i}, s\right)$ should be viewed as the zeta function of a relative $H^{i}$ of $f$ in the sense of a motivic (i.e., perverse) $t$-structure, ${ }^{1}$ and $\zeta\left(H^{1}, s\right)$ differs from the Hasse-Weil $L$-function of $J_{F}$ by finitely many Euler factors. Our conjecture is equivalent to the statements

$$
\operatorname{ord}_{s=1} \zeta\left(H^{1}, s\right)=\operatorname{rank}_{\mathbb{Z}} \operatorname{Pic}^{0}(\mathcal{X})
$$

and

$$
\zeta^{*}\left(H^{1}, 1\right)=\frac{\# \operatorname{Br}(\overline{\mathcal{X}}) \cdot \delta^{2} \cdot \Omega(\mathcal{X}) \cdot R(\mathcal{X})}{\left(\#\left(\operatorname{Pic}^{0}(\mathcal{X})_{\text {tor }} / \operatorname{Pic}\left(\mathcal{O}_{F}\right)\right)\right)^{2}} \cdot \prod_{v \text { real }} \frac{\# \Phi_{v}}{\delta_{v}^{\prime} \delta_{v}},
$$

where $\operatorname{Pic}^{0}(\mathcal{X})$ is the kernel of the degree map on $\operatorname{Pic}(\mathcal{X}), R(\mathcal{X})$ is the regulator of a certain intersection pairing on $\operatorname{Pic}^{0}(\mathcal{X})$ and $\Omega(\mathcal{X})$ is the determinant of the period isomorphism between the finitely generated abelian groups $H^{1}(\mathcal{X}(\mathbb{C}), 2 \pi i \cdot \mathbb{Z})^{G_{\mathbb{R}}}$ and $H^{1}\left(\mathcal{X}, \mathcal{O}_{\mathcal{X}}\right)$. The integer $\delta$ is the index of $\mathcal{X}_{F}$ - that is, the greatest common divisor of the degrees of all closed points. Furthermore, $\Phi_{v}=J_{F}\left(F_{v}\right) / J_{F}\left(F_{v}\right)^{0}$ is the group of components of the

${ }^{1}$ For the formal definition of $\zeta\left(H^{i}, s\right)$, we refer to Lemma 16. Note that our indexing $H^{0}, H^{1}, H^{2}$ does not conform to that of the perverse $t$-structure (discussed in Remark 5 ), but it matches the indexing on the generic fibre and hence is more intuitive. 
group of $F_{v}$-rational points of $J_{F}$, and $\delta_{v}^{\prime}$ (resp., $\delta_{v}$ ) are the period (resp., index) of $\mathcal{X}_{F_{v}}$ over $F_{v}$. The group $\operatorname{Br}(\overline{\mathcal{X}})$ coincides with $\operatorname{Br}(\mathcal{X})$ if $F$ has no real places and differs from $\operatorname{Br}(\mathcal{X})$ by a 2-torsion group in general. The group $\operatorname{Br}(\overline{\mathcal{X}})$ is naturally self-dual, and the quantity $\# \operatorname{Br}(\overline{\mathcal{X}}) \delta^{2}$ will also arise as the cardinality of a naturally self-dual group in our computation (one could call this group the $H^{1}$-part of the Brauer group; see Lemma 8).

We then prove that our conjecture (equations (5) and (6)) is equivalent to the Birch and Swinnerton-Dyer conjecture for the Jacobian of $\mathcal{X}_{F}$, provided that our intersection pairing agrees with the Arakelov intersection pairing. This result was shown for $f$ smooth in [6, Theorem 5.27] but only rather indirectly, via compatibility of both conjectures with the Tamagawa number conjecture. Here we give a direct proof without assumptions on $f$ (such as existence of a section, which would simplify the proof considerably). If $\mathcal{X}$ is a smooth projective surface over a finite field, fibred over a curve $f: \mathcal{X} \rightarrow S$, the equivalence of the Birch and Swinnerton-Dyer formula for the Jacobian of the generic fibre with a special-value conjecture for the zeta function $\zeta\left(H_{a b s}^{2}, s\right)$ of the absolute $H^{2}$-motive of $\mathcal{X}$ has been much studied in the literature, going back to the article of Tate [35] (see also [11]). At least for suitable choices of $f$, the two functions $\zeta\left(H^{1}, s\right)$ and $\zeta\left(H_{a b s}^{2}, s\right)$ only differ by a very simple Euler factor (see Remark 5 ), and it seems likely that our methods will also give a simplification of the arguments in [11]. To the best of our knowledge, our special-value conjecture is the first such for arithmetic surfaces of characteristic 0 , and in this context one of course does not have an analogue of $H_{a b s}^{2}$.

Combined with the analytic class number formula for the Dedekind zeta function $\zeta_{F}(s)$ at $s=1$ and $s=0$, formula (6) becomes

$$
\zeta^{*}(\mathcal{X}, 1)=\frac{2^{r_{1}}(2 \pi)^{r_{2}}}{\Omega(\mathcal{X}) \sqrt{\left|D_{F}\right|}} \cdot \frac{\left(\# \operatorname{Pic}^{0}(\mathcal{X})\right)_{t o r}^{2}}{\# \operatorname{Br}(\overline{\mathcal{X}}) \delta^{2}\left(\# \mu_{F}\right)^{2}} \cdot \frac{R_{F}^{2}}{R(\mathcal{X})} \cdot \prod_{v \text { real }} \frac{\delta_{v}^{\prime} \delta_{v}}{\# \Phi_{v}}
$$

Since there are now examples of elliptic curves $E / \mathbb{Q}$ for which $\amalg(E)$ is known to be finite and the Birch and Swinnerton-Dyer conjecture is completely proven (see [37, Theorem 9.3] and [21, Theorem 1.2]), our conjectures (5), (6) and (7) hold for any regular model $\mathcal{X}$ of any principal homogenous space of any elliptic curve isogenous over $\mathbb{Q}$ to such an $E$. In Remark 4 we also verify our conjectures directly if $\mathcal{X}_{F}$ has genus 0 .

We give a brief summary of each section of this paper. In Section 2 we prove that $C(\mathcal{X}, 1)=1$ for general $\mathcal{X}$. Since $\mathbb{Z}(1)=\mathbb{G}_{m}[-1]$, the proof involves elementary properties of the sheaf $\mathbb{G}_{m}$ in the étale topology. However, the $p$-part of $C(\mathcal{X}, 1)$ is defined in $[6$, Definition 5.6] via the eh-topology on schemes over $\mathbb{F}_{p}[9]$, and our proof eventually reduces to a curious statement - Proposition 2.1 - which in some sense says that the difference between étale and eh-cohomology is the same for the sheaves $\mathbb{G}_{m}$ and $\mathcal{O}$. In order to prove Proposition 2.1, we develop some results on the eh-topology which might be of independent interest.

In Section 3 we prove, for an arithmetic surface $\mathcal{X}$, Conjecture $\mathbf{A V}(\mathcal{X}, 1)$ of $[6$, Conjecture 6.23] using results of Saito [30]. This conjecture is necessary to define the Weil-étale complexes in terms of which our special value conjecture is formulated. 
In Section 4 we define Weil-étale complexes associated to the relative $H^{1}$-motive of $f$ and then translate our special-value conjecture for $\zeta\left(H^{1}, s\right)$ from the formulation in terms of a fundamental line to the explicit form (6) already given.

In Section 5 we slightly generalise a result due to Geisser [10] which relates the cardinalities of $\operatorname{Br}(\mathcal{X})$ and $\amalg\left(J_{F}\right)$. More precisely, working with sheaves on the ArtinVerdier compactification $\bar{S}$ of $S$ [6, Appendix A], we give a different proof of Geisser's formula in order to generalise it from totally imaginary to arbitrary base number fields $F$.

Finally, in Section 6 we prove the equivalence to the Birch and Swinnerton-Dyer conjecture. Besides Geisser's formula, the key ingredients in the proof of our main Theorem 6.1 are Lemma 15 on the behavior of intersection pairings in exact sequences, results of Bosch and Liu [3] on component groups of Néron models and results of Liu, Lorenzini and Raynaud [23] on tangent spaces of Néron models.

\section{Convention}

In this paper we shall define many complexes as mapping cones of morphisms in the derived category of sheaves on some site - that is, the derived category of some Grothendieck abelian category $\mathscr{A}$. These shall be understood in an $\infty$-categorial sense without further mention. The (stable) derived $\infty$-category $\mathscr{D}(\mathscr{A})$ was defined in [25, Definition 1.3.5.8], and by [25, Proposition 1.3.5.15], any chain complex of objects of $\mathscr{A}$ (resp., any map of complexes) gives an object (resp., morphism) of $\mathscr{D}(\mathscr{A})$. A commutative diagram consists not only of the morphisms (1-cells) which we shall actually write down but also of 2-cells (and higher cells, for example in diagram (52)) witnessing commutativity which we leave implicit. By an exact triangle we mean a cofibre sequence [25, Definition 1.1.6], and by a mapping cone, a cofibre. By [25, Remark 1.1.1.7], the space of cofibres of a given morphism is a contractible Kan complex - that is, the cofibre is an object defined up to unique isomorphism in the classical derived category $h \mathscr{D}(\mathscr{A})$. The universal property of the cofibre as a $\infty$-categorical colimit also gives the existence of nine-term diagrams such as diagram (47) from the input data of the top left square.

\section{The correction factor for $n=1$}

We recall some definitions from [6]. Throughout this paper we use the notation

$$
C / M:=C \otimes_{\mathbb{Z}}^{L} \mathbb{Z} / M \mathbb{Z}
$$

for the mapping cone of multiplication by $M$, where $C$ is a complex of sheaves on some site and $M$ is an integer. For any scheme $Z$ and integer $n$, we denote by $\mathbb{Z}(n)=z^{n}(-, 2 n-\bullet)$ Bloch's higher Chow complex, viewed as a complex of sheaves on the small étale site of $Z$. For any prime number $p$ we set

$$
R \Gamma\left(Z, \mathbb{Z}_{p}(n)\right):=R \Gamma\left(Z_{e t}, \mathbb{Z}_{p}(n)\right):=R \underset{\nu}{\lim _{\nu}} R \Gamma\left(Z_{e t}, \mathbb{Z}(n) / p^{\nu}\right)
$$

and

$$
R \Gamma\left(Z, \mathbb{Q}_{p}(n)\right):=R \Gamma\left(Z, \mathbb{Z}_{p}(n)\right) \otimes_{\mathbb{Z}_{p}} \mathbb{Q}_{p}
$$


For $\mathcal{X}$ regular, proper over $\operatorname{Spec}(\mathbb{Z})$ and $n \in \mathbb{Z}$ we consider the derived de Rham complex modulo the Hodge filtration $L \Omega_{\mathcal{X} / \mathbb{Z}}^{*} / \mathrm{Fil}^{n}$ (see [20, Section VIII.2.1]) as a complex of abelian sheaves on the Zariski site of $\mathcal{X}$. We obtain a perfect complex of abelian groups

$$
R \Gamma_{d R}(\mathcal{X} / \mathbb{Z}) / F^{n}:=R \Gamma\left(\mathcal{X}_{Z a r}, L \Omega_{\mathcal{X} / \mathbb{Z}}^{*} / \mathrm{Fil}^{n}\right)
$$

and its base change $R \Gamma_{d R}\left(\mathcal{X}_{A} / A\right) / F^{n}$ to any ring $A$.

Let $\left(\operatorname{Sch}^{d} / \mathbb{F}_{p}\right)_{e h}$ be the category of separated, finite-type schemes $Z / \mathbb{F}_{p}$ of dimension $\leq d$ with the eh-topology [9, Definition 2.1], and $\left(\mathrm{Sm}^{d} / \mathbb{F}_{p}\right)$ et be the full subcategory of smooth schemes with the étale topology. There is a natural pair of adjoint functors on abelian sheaves [9, Lemma 2.5]

$$
\left(\rho_{d}^{*}, \rho_{d, *}\right): \mathrm{Ab}\left(\mathrm{Sch}^{d} / \mathbb{F}_{p}\right)_{e h}^{\sim} \rightarrow \mathrm{Ab}\left(\mathrm{Sm}^{d} / \mathbb{F}_{p}\right)_{e t}^{\sim},
$$

and we set

$$
\begin{aligned}
& R \Gamma\left(Z_{e h}, \mathbb{Z}(n)\right):=R \Gamma\left(Z_{e h}, \rho_{d}^{*} \mathbb{Z}(n)^{S V}\right),
\end{aligned}
$$

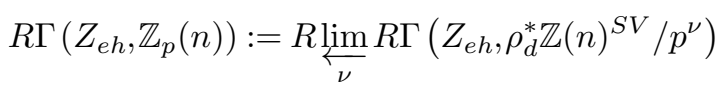

and

$$
R \Gamma\left(Z_{e h}, \mathbb{Q}_{p}(n)\right):=R \Gamma\left(Z_{e h}, \mathbb{Z}_{p}(n)\right) \otimes_{\mathbb{Z}_{p}} \mathbb{Q}_{p},
$$

where $\mathbb{Z}(n)^{S V}$ is the Suslin-Voevodsky motivic complex on the smooth site $\mathrm{Sm} / \mathbb{F}_{p}$. For smooth $Z / \mathbb{F}_{p}$ there is a quasi-isomorphism $\mathbb{Z}(n) \cong \mathbb{Z}(n)^{S V}$ on the small étale site of $Z$, but $\mathbb{Z}(n)$ is not functorial for arbitrary morphisms in $\mathrm{Sm} / \mathbb{F}_{p}$.

Various results on the eh-topology in [9] are only valid under a resolution-of-singularities assumption $R\left(\mathbb{F}_{p}, d\right)$ for varieties of dimension $\leq d[9$, Definition 2.4], and we shall indicate along the way which of our results depend on it. For example, if one assumes $R\left(\mathbb{F}_{p}, d\right)$, then $\rho_{d}^{*}$ is exact; $R\left(\mathbb{F}_{p}, d\right)$ holds for $d \leq 2$.

Under $R\left(\mathbb{F}_{p}, d\right)$ and for smooth $Z / k$, there is an isomorphism [9, Theorem 4.3]

$$
R \Gamma\left(Z_{e h}, \rho_{d}^{*} \mathbb{Z}(n)^{S V}\right) \cong R \Gamma\left(Z_{e t}, \mathbb{Z}(n)^{S V}\right) \cong R \Gamma\left(Z_{e t}, \mathbb{Z}(n)\right) .
$$

Fix a prime number $p$ and let $\mathcal{X}$ be regular, proper and flat over $\operatorname{Spec}(\mathbb{Z})$. The following is [6, Conjecture 5.5]:

Conjecture $1\left(\mathbf{D}_{p}(\mathcal{X}, n)\right)$. There is an exact triangle of complexes of $\mathbb{Q}_{p}$-vector spaces

$$
R \Gamma_{d R}\left(\mathcal{X}_{\mathbb{Q}_{p}} / \mathbb{Q}_{p}\right) / F^{n}[-1] \rightarrow R \Gamma\left(\mathcal{X}_{\mathbb{Z}_{p}, e t}, \mathbb{Q}_{p}(n)\right) \rightarrow R \Gamma\left(\mathcal{X}_{\mathbb{F}_{p}, e h}, \mathbb{Q}_{p}(n)\right) .
$$

This conjecture gives an isomorphism

$$
\begin{aligned}
\lambda_{p}=\lambda_{p}(\mathcal{X}, n) & :\left(\operatorname{det}_{\mathbb{Z}_{p}} R \Gamma\left(\mathcal{X}_{\mathbb{Z}_{p}, e t}, \mathbb{Z}_{p}(n)\right)\right)_{\mathbb{Q}_{p}} \\
& \stackrel{\sim}{\longrightarrow} \operatorname{det}_{\mathbb{Q}_{p}} R \Gamma\left(\mathcal{X}_{\mathbb{Z}_{p}, e t}, \mathbb{Q}_{p}(n)\right) \\
& \stackrel{\sim}{\rightarrow} \operatorname{det}_{\mathbb{Q}_{p}} R \Gamma\left(\mathcal{X}_{\mathbb{F}_{p}, e h}, \mathbb{Q}_{p}(n)\right) \otimes_{\mathbb{Q}_{p}} \operatorname{det}_{\mathbb{Q}_{p}}^{-1} R \Gamma_{d R}\left(\mathcal{X}_{\mathbb{Q}_{p}} / \mathbb{Q}_{p}\right) / F^{n} \\
& \stackrel{\sim}{\longrightarrow}\left(\operatorname{det}_{\mathbb{Z}_{p}} R \Gamma\left(\mathcal{X}_{\mathbb{F}_{p}, e h}, \mathbb{Z}_{p}(n)\right) \otimes_{\mathbb{Z}_{p}} \operatorname{det}_{\mathbb{Z}_{p}}^{-1} R \Gamma_{d R}\left(\mathcal{X}_{\mathbb{Z}_{p}} / \mathbb{Z}_{p}\right) / F^{n}\right)_{\mathbb{Q}_{p}},
\end{aligned}
$$


and we define

$$
d_{p}(\mathcal{X}, n) \in \mathbb{Q}_{p}^{\times} / \mathbb{Z}_{p}^{\times}
$$

such that

$$
\begin{aligned}
& \lambda_{p}\left(d_{p}(\mathcal{X}, n)^{-1} \cdot \operatorname{det}_{\mathbb{Z}_{p}} R \Gamma\left(\mathcal{X}_{\mathbb{Z}_{p}, e t}, \mathbb{Z}_{p}(n)\right)\right) \\
= & \operatorname{det}_{\mathbb{Z}_{p}} R \Gamma\left(\mathcal{X}_{\mathbb{F}_{p}, e h}, \mathbb{Z}_{p}(n)\right) \otimes_{\mathbb{Z}_{p}} \operatorname{det}_{\mathbb{Z}_{p}}^{-1} R \Gamma_{d R}\left(\mathcal{X}_{\mathbb{Z}_{p}} / \mathbb{Z}_{p}\right) / F^{n} .
\end{aligned}
$$

We then set

$$
\chi\left(\mathcal{X}_{\mathbb{F}_{p}}, \mathcal{O}, n\right):=\sum_{i \leq n, j}(-1)^{i+j} \cdot(n-i) \cdot \operatorname{dim}_{\mathbb{F}_{p}} H^{j}\left(\mathcal{X}_{\mathbb{F}_{p}, e h}, \rho_{d}^{*} \Omega^{i}\right),
$$

where $\Omega^{i}$ is the sheaf of Kaehler differentials on $\operatorname{Sm} / \mathbb{F}_{p}$. Under $R\left(\mathbb{F}_{p}, d\right)$ and for smooth $Z / \mathbb{F}_{p}$, there is an isomorphism [9, Theorem 4.7]

$$
H^{i}\left(Z_{e t}, \Omega^{i}\right) \cong H^{i}\left(Z_{e h}, \rho_{d}^{*} \Omega^{i}\right) .
$$

We finally set

$$
c_{p}(\mathcal{X}, n):=p^{\chi\left(\mathcal{X}_{\mathbb{F}_{p}}, \mathcal{O}, n\right)} \cdot d_{p}(\mathcal{X}, n)
$$

and

$$
C(\mathcal{X}, n):=\prod_{p<\infty}\left|c_{p}(\mathcal{X}, n)\right|_{p}
$$

Conjecture 2. Let $\mathcal{X}$ be regular of dimension $d$, proper and flat over $\operatorname{Spec}(\mathbb{Z})$, and assume $R\left(\mathbb{F}_{p}, d-1\right)$. Then Conjecture $\mathbf{D}_{p}(\mathcal{X}, 1)$ holds and we have $C(\mathcal{X}, 1)=1$.

Proof. Fix a prime number $p$. Then the base change $\mathcal{X}_{\mathbb{Z}_{p}}$ is again a regular scheme. For $n=1$ we have isomorphisms $\mathbb{Z}(1) \cong \mathbb{G}_{m}[-1]$ on $\mathcal{X}_{\mathbb{Z}_{p}, \text { et }}$ and $\mathbb{Z}(1)^{S V} \cong \mathbb{G}_{m}[-1]$ on $\mathrm{Sm} / \mathbb{F}_{p}$. There are an exact sequence of coherent sheaves

$$
0 \rightarrow \mathcal{I} \rightarrow \mathcal{O}_{\mathcal{X}_{\mathbb{Z}_{p}}} \rightarrow i_{*} \mathcal{O}_{\mathcal{X}_{\mathbb{F}_{p}}} \rightarrow 0
$$

and an exact sequence of abelian sheaves

$$
0 \rightarrow 1+\mathcal{I} \rightarrow \mathbb{G}_{m, \mathcal{X}_{\mathbb{Z}_{p}}} \rightarrow i_{*} \mathbb{G}_{m, \mathcal{X}_{\mathbb{F}_{p}}} \rightarrow 0
$$

on $\mathcal{X}_{\mathbb{Z}_{p}, e t}$. Here $1+\mathcal{I}$ is just our notation for the kernel; the sections of this sheaf over an étale $U \rightarrow \mathcal{X}_{\mathbb{Z}_{p}}$ need not coincide with $1+\mathcal{I}(U)$. Passing to $p$-adic completions and shifting by $[-1]$, we obtain an exact triangle

$$
R \underset{\nu}{\lim } R \Gamma\left(\mathcal{X}_{\mathbb{Z}_{p}, e t},(1+\mathcal{I}) / p^{\nu}\right)[-1] \rightarrow R \Gamma\left(\mathcal{X}_{\mathbb{Z}_{p}, e t}, \mathbb{Z}_{p}(1)\right) \rightarrow R \Gamma\left(\mathcal{X}_{\mathbb{F}_{p}, e t}, \mathbb{Z}_{p}(1)\right) \rightarrow
$$

For $n=1$, there is an isomorphism $L \Omega_{\mathcal{X} / \mathbb{Z}}^{*} / \mathrm{Fil}^{1} \cong \mathcal{O}_{\mathcal{X}}$ and definition (9) specialises to

$$
\chi\left(\mathcal{X}_{\mathbb{F}_{p}}, \mathcal{O}, 1\right)=\chi\left(\mathcal{X}_{\mathbb{F}_{p}}, \mathcal{O}\right):=\sum_{j}(-1)^{j} \cdot \operatorname{dim}_{\mathbb{F}_{p}} H^{j}\left(\mathcal{X}_{\mathbb{F}_{p}, e h}, \rho_{d}^{*} \mathcal{O}\right)
$$


The outer terms in expression (10) are computed by, respectively, Lemma 1 and Proposition 2.1. Hence we obtain the exact triangle

$$
R \Gamma\left(\mathcal{X}_{\mathbb{Q}_{p}}, \mathcal{O}_{\mathcal{X}_{\mathbb{Q}_{p}}}\right)[-1] \rightarrow R \Gamma\left(\mathcal{X}_{\mathbb{Z}_{p}, e t}, \mathbb{Q}_{p}(1)\right) \rightarrow R \Gamma\left(\mathcal{X}_{\mathbb{F}_{p}, e h}, \mathbb{Q}_{p}(1)\right)
$$

of Conjecture $\mathbf{D}_{p}(\mathcal{X}, 1)$ after scalar extension to $\mathbb{Q}_{p}$. Moreover, Lemma 1 and Proposition 2.1 show that

$$
\begin{aligned}
\lambda_{p}\left(\operatorname{det}_{\mathbb{Z}_{p}} R \Gamma\left(\mathcal{X}_{\mathbb{Z}_{p}, e t}, \mathbb{Z}_{p}(1)\right)\right)= & p^{\chi_{e t}\left(\mathcal{X}_{\mathbb{F}_{p}}, \mathcal{O}\right)-\chi\left(\mathcal{X}_{\mathbb{F}_{p}}, \mathcal{O}\right)} \cdot \operatorname{det}_{\mathbb{Z}_{p}} R \Gamma\left(\mathcal{X}_{\mathbb{F}_{p}, e h}, \mathbb{Z}_{p}(1)\right) \\
& \otimes_{\mathbb{Z}_{p}} p^{-\chi_{e t}\left(\mathcal{X}_{\mathbb{F}_{p}}, \mathcal{O}\right)} \cdot \operatorname{det}_{\mathbb{Z}_{p}}^{-1} R \Gamma\left(\mathcal{X}_{\mathbb{Z}_{p}}, \mathcal{O}_{\mathcal{X}_{\mathbb{Z}_{p}}}\right)
\end{aligned}
$$

inside $\operatorname{det}_{\mathbb{Q}_{p}} R \Gamma\left(\mathcal{X}_{\mathbb{Z}_{p}, e t}, \mathbb{Q}_{p}(1)\right)$ - that is, we have $d_{p}(\mathcal{X}, 1)=p^{-\chi\left(\mathcal{X}_{\mathbb{F}_{p}}, \mathcal{O}\right)}$ and therefore $c_{p}(\mathcal{X}, 1)=1$. This finishes the proof.

Lemma 1. There is an isomorphism

$$
R \varliminf_{\nu} \lim _{\nu} R \Gamma\left(\mathcal{X}_{\mathbb{Z}_{p}, e t},(1+\mathcal{I}) / p^{\nu}\right) \otimes_{\mathbb{Z}_{p}} \mathbb{Q}_{p} \cong R \Gamma\left(\mathcal{X}_{\mathbb{Q}_{p}}, \mathcal{O}_{\mathcal{X}_{\mathbb{Q}_{p}}}\right)
$$

so that

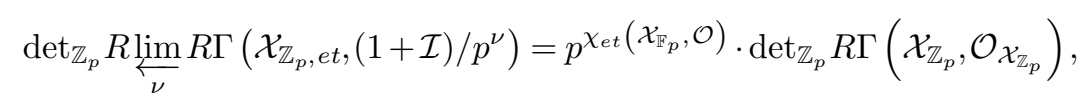

where

$$
\chi_{e t}\left(\mathcal{X}_{\mathbb{F}_{p}}, \mathcal{O}\right):=\sum_{j}(-1)^{j} \cdot \operatorname{dim}_{\mathbb{F}_{p}} H^{j}\left(\mathcal{X}_{\mathbb{F}_{p}, e t}, \mathcal{O}\right)
$$

is the analogue of definition (11) for the étale (or Zariski) topology.

Proof. Let $\left(\mathfrak{X}, \mathcal{O}_{\mathfrak{X}}\right)$ be the formal completion of $\mathcal{X}_{\mathbb{Z}_{p}}$ at the ideal $\mathcal{I}=(p)$. The underlying topological space of $\mathfrak{X}$ is $\mathcal{X}_{\mathbb{F}_{p}}$, and we denote by $i: \mathfrak{X} \rightarrow \mathcal{X}_{\mathbb{Z}_{p}}$ the natural morphism of ringed spaces and étale topoi. We have an exact sequence on $\mathfrak{X}_{\text {et }}$

$$
0 \rightarrow 1+\mathcal{I}_{\mathfrak{X}} \rightarrow \mathbb{G}_{m, \mathfrak{X}} \rightarrow \mathbb{G}_{m, \mathcal{X}_{p}} \rightarrow 0 .
$$

Lemma 2. For any $\nu \geq 1$, the natural morphism $i^{*}(1+\mathcal{I}) \rightarrow 1+\mathcal{I}_{\mathfrak{X}}$ induces an isomorphism on $\mathfrak{X}_{\text {et }}$

$$
i^{*}(1+\mathcal{I}) / p^{\nu} \rightarrow\left(1+\mathcal{I}_{\mathfrak{X}}\right) / p^{\nu}
$$

Proof. Recall that formula (14) is meant in the derived sense, so we have to investigate the kernel and cokernel of multiplication by $p^{\nu}$ separately. For each (connected) étale $\operatorname{Spec}(A) \rightarrow \mathcal{X}_{\mathbb{Z}_{p}}$, the map on kernels is

$$
\mu_{p^{\nu}}(A) \cap(1+\mathcal{I})(A) \rightarrow \mu_{p^{\nu}}(\hat{A}) \cap\left(1+\mathcal{I}_{\mathfrak{X}}\right)(\hat{A}),
$$

where $\hat{A}$ denotes the $p$-adic completion. Now any element of $\mu_{p^{\nu}}(A)$ lies in the integral closure of $\mathbb{Z}_{p}$ in $A$, which coincides with the integral closure of $\mathbb{Z}_{p}$ in the fraction field 
$K$ of $A$, as $A$ is regular. Hence $\mu_{p^{\nu}}(A)$ is contained in the algebraic closure $L$ of $\mathbb{Q}_{p}$ in $K$. But $L / \mathbb{Q}_{p}$ is finite, as $\mathcal{X}_{\mathbb{Z}_{p}} \rightarrow \operatorname{Spec}\left(\mathbb{Z}_{p}\right)$ is of finite type. We conclude that $\mu_{p^{\nu}}(A)$ is contained in $\mathcal{O}_{L}$, which is already $p$-adically complete - that is, we have $\mu_{p^{\nu}}(\hat{A})=\mu_{p^{\nu}}(A)$. Hence map (15) is also an isomorphism (in fact, both sides vanish unless $p=2$ ).

Concerning the cokernel of $p^{\nu}$, we first note that for each étale $\operatorname{Spec}(A) \rightarrow \mathcal{X}_{\mathbb{Z}_{p}}$ we have $\left(1+\mathcal{I}_{\mathfrak{X}}\right)(\hat{A})=1+\mathcal{I}_{\mathfrak{X}}(\hat{A})$, since the inverse of any element $1+p \cdot x$ is given by a geometric series. Moreover, the usual power series of the exponential and the logarithm induce an isomorphism

$$
\log : 1+\mathcal{I}_{\mathfrak{X}}^{\nu}(\hat{A})=1+p^{\nu} \cdot \hat{A} \cong p^{\nu} \cdot \hat{A}=\mathcal{I}_{\mathfrak{X}}^{\nu}(\hat{A})
$$

for each $\nu \geq 1(\nu \geq 2$ if $p=2)$. In particular, we conclude that any element in $1+p^{\nu+2} \cdot \hat{A}$ has a $p^{\nu}$ th $\operatorname{root}$ in $1+p \cdot \hat{A}$. Given $1+x \in 1+p \cdot \hat{A}$ and $\nu \geq 1$, we can write

$$
1+x=1+x_{0}+p^{\nu+2} \cdot x_{1}=\left(1+x_{0}\right) \cdot\left(1+p^{\nu+2} \frac{x_{1}}{1+x_{0}}\right),
$$

with $x_{0} \in \mathcal{I}(A)=p \cdot A$ and $x_{1} \in \hat{A}$, and we can also assume that $1+x_{0} \in A^{\times}$, since inverting $1+x_{0}$ does not remove any points of $\mathcal{X}_{\mathbb{F}_{p}}=\mathfrak{X}$. Hence we find $1+x \in(1+\mathcal{I})(A)$. $\left(1+\mathcal{I}_{\mathfrak{X}}(\hat{A})\right)^{p^{\nu}}$ - that is, that the map (14) is a surjection on cokernels of multiplication by $p^{\nu}$.

To show that it is also an injection, we need to consider an element $1+x_{0} \in(1+\mathcal{I})(A)$ that becomes a $p^{\nu}$ th power in $1+\mathcal{I}_{\mathfrak{X}}(\hat{B})$ for some étale neighbourhood $\operatorname{Spec}(B) \rightarrow$ $\operatorname{Spec}(A)$ of any given point $\mathfrak{p} \in \operatorname{Spec}(A /(p))$ and show that $1+x_{0} \in\left(1+\mathcal{I}\left(B^{\prime}\right)\right)^{p^{\nu}}$ in some étale neighbourhood $\operatorname{Spec}\left(B^{\prime}\right) \rightarrow \operatorname{Spec}(B)$ of $\mathfrak{p}$. Since $1+x_{0} \in\left(1+\mathcal{I}_{\mathfrak{X}}(\hat{B})\right)^{p^{\nu}}$, we have $1+x_{0}=1+p^{\nu+1} \cdot y_{0}$. Pick a prime $\mathfrak{q}$ of $B$ above $\mathfrak{p}$. If $y_{0} \in \mathfrak{q}$, we can write

$$
1+p^{\nu+1} \cdot y_{0}=\frac{1+p^{\nu+1} \cdot\left(y_{0}+1\right)}{1+p^{\nu+1} \cdot\left(1+p^{\nu+1} \cdot y_{0}\right)^{-1}}
$$

where neither $y_{0}+1$ nor $\left(1+p^{\nu+1} \cdot y_{0}\right)^{-1}$ lies in $\mathfrak{q}$. Hence we can assume $y_{0} \notin \mathfrak{q}$, and in fact $y_{0} \in B^{\times}$. We then adjoin an element $z$ satisfying the integral equation

$$
f(z):=\frac{(z+p)^{p^{\nu}}}{p^{\nu+1}}-\frac{z^{p^{\nu}}}{p^{\nu+1}}-z^{p^{\nu}} \cdot y_{0}=\frac{z^{p^{\nu}}}{p^{\nu+1}}\left[\left(1+\frac{p}{z}\right)^{p^{\nu}}-\left(1+p^{\nu+1} \cdot y_{0}\right)\right]=0
$$

over $B$. Then $B^{\prime}=B\left[z, \frac{1}{z}\right]$ contains a $p^{\nu}$ th root of $1+p^{\nu+1} \cdot y_{0}$ and

$$
\operatorname{Spec}\left(B\left[z, \frac{1}{z}\right]\right) \rightarrow \operatorname{Spec}(B)
$$

is étale and surjective. Indeed, it is clearly étale at all primes $\mathfrak{q}^{\prime} \in \operatorname{Spec}(B)$ with $p \notin \mathfrak{q}^{\prime}$, and if $p \in \mathfrak{q}^{\prime}$, we have (modulo $\mathfrak{q}^{\prime}$ ) 


$$
f(z) \equiv \begin{cases}z^{p^{\nu}-1}+\left(p^{\nu}-1\right) z^{p^{\nu}-2}-z^{p^{\nu}} \cdot y_{0} \equiv z^{p^{\nu}-2}\left(1+z+z^{2} \cdot y_{0}\right) & \text { if } p=2 \\ z^{p^{\nu}-1}-z^{p^{\nu}} \cdot y_{0}=z^{p^{\nu}-1}\left(1-z \cdot y_{0}\right) & \text { if } p \neq 2\end{cases}
$$

so inverting $z$ removes all primes where $\mathfrak{q}^{\prime}$ is ramified. Moreover, there is a prime $B\left[z, \frac{1}{z}\right] \cdot \mathfrak{q}^{\prime}$ above $\mathfrak{q}^{\prime}$ with a finite separable residue field extension (in fact, the same residue field if $p$ is odd).

Since $(1+\mathcal{I}) / p^{\nu}$ has torsion cohomology, proper base change gives an isomorphism

$$
R \Gamma\left(\mathcal{X}_{\mathbb{Z}_{p}, e t},(1+\mathcal{I}) / p^{\nu}\right) \cong R \Gamma\left(\mathcal{X}_{\mathbb{F}_{p}, e t}, i^{*}(1+\mathcal{I}) / p^{\nu}\right)
$$

and Lemma 2 then gives an isomorphism

$$
R{\underset{\nu}{\nu}}_{\lim _{\nu}} R\left(\mathcal{X}_{\mathbb{Z}_{p}, e t},(1+\mathcal{I}) / p^{\nu}\right) \cong R \Gamma\left(\mathfrak{X}_{e t}, 1+\mathcal{I}_{\mathfrak{X}}\right)
$$

since $1+\mathcal{I}_{\mathfrak{X}}$ is already $p$-adically complete. Consider the following diagram with exact rows, where the vertical isomorphism is induced by logarithm (16) for $k$ large enough (in fact $k \geq 2)$ :

$$
\begin{aligned}
& R \Gamma\left(\mathfrak{X}_{e t}, 1+\mathcal{I}_{\mathfrak{X}}^{k}\right) \stackrel{\alpha}{\longrightarrow} R \Gamma\left(\mathfrak{X}_{e t}, 1+\mathcal{I}_{\mathfrak{X}}\right) \longrightarrow R \Gamma\left(\mathfrak{X}_{e t}, 1+\mathcal{I}_{\mathfrak{X}} / 1+\mathcal{I}_{\mathfrak{X}}^{k}\right) \longrightarrow \\
& \log \downarrow \sim \\
& R \Gamma\left(\mathfrak{X}_{e t}, \mathcal{I}_{\mathfrak{X}}^{k}\right) \\
& \downarrow \sim \\
& R \Gamma\left(\mathfrak{X}, \mathcal{I}_{\mathfrak{X}}^{k}\right) \stackrel{\alpha^{a d d}}{\longrightarrow} R \Gamma\left(\mathfrak{X}, \mathcal{O}_{\mathfrak{X}}\right) \quad \longrightarrow \quad R \Gamma\left(\mathfrak{X}, \mathcal{O}_{\mathfrak{X}} / \mathcal{I}_{\mathfrak{X}}^{k}\right) \quad \longrightarrow .
\end{aligned}
$$

We have isomorphisms of abelian sheaves

$$
\mathcal{I}_{\mathfrak{X}}^{i} / \mathcal{I}_{\mathfrak{X}}^{i+1} \cong\left(1+\mathcal{I}_{\mathfrak{X}}^{i}\right) /\left(1+\mathcal{I}_{\mathfrak{X}}^{i+1}\right), \quad x \mapsto 1+x,
$$

and $R \Gamma\left(\mathfrak{X}, \mathcal{I}_{\mathfrak{X}}^{i} / \mathcal{I}_{\mathfrak{X}}^{i+1}\right)$ is a perfect complex of $\mathbb{F}_{p}$-modules, since $\mathfrak{X}$ is proper. It follows that the maps $\alpha$ and $\alpha^{a d d}$ are isomorphisms after tensoring with $\mathbb{Q}_{p}$. Finally, recall the theorem of formal functions [17, Theorem III.11.1]:

$$
R \Gamma\left(\mathfrak{X}, \mathcal{O}_{\mathfrak{X}}\right) \cong R \Gamma\left(\mathcal{X}_{\mathbb{Z}_{p}}, \mathcal{O}_{\mathcal{X}_{\mathbb{Z}_{p}}}\right) .
$$

Isomorphism (12) is then the composition of the scalar extensions to $\mathbb{Q}_{p}$ of isomorphism (17), $\alpha, \log , \alpha^{a d d}$ and expression (19). The diagram and formula (18) show that under this isomorphism, we have

$$
\begin{aligned}
\operatorname{det}_{\mathbb{Z}_{p}} R{\underset{\nu}{\lim }}_{\nu} R \Gamma\left(\mathcal{X}_{\mathbb{Z}_{p}, e t},(1+\mathcal{I}) / p^{\nu}\right) \\
=p^{-\chi\left(1+\mathcal{I}_{\mathfrak{X}} / 1+\mathcal{I}_{\mathfrak{X}}^{k}\right)+\chi\left(\mathcal{O}_{\mathfrak{X}} / \mathcal{I}_{\mathfrak{X}}^{k}\right)} \cdot \operatorname{det}_{\mathbb{Z}_{p}} R \Gamma\left(\mathcal{X}_{\left.\mathbb{Z}_{p}, \mathcal{O}_{\mathcal{X}_{\mathbb{Z}_{p}}}\right)}\right. \\
=p^{\chi\left(\mathcal{O}_{\mathfrak{X}} / \mathcal{I}_{\mathfrak{X}}\right)} \cdot \operatorname{det}_{\mathbb{Z}_{p}} R \Gamma\left(\mathcal{X}_{\mathbb{Z}_{p}}, \mathcal{O}_{\mathcal{X}_{\mathbb{Z}_{p}}}\right)=p^{\chi_{e t}\left(\mathcal{X}_{\mathbb{F}_{p}}, \mathcal{O}\right)} \cdot \operatorname{det}_{\mathbb{Z}_{p}} R \Gamma\left(\mathcal{X}_{\mathbb{Z}_{p}}, \mathcal{O}_{\mathcal{X}_{\mathbb{Z}_{p}}}\right) .
\end{aligned}
$$




\subsection{Results on the eh-topology}

To prepare for the proof of Proposition 2.1, we develop some results on the eh-topology which might be of independent interest. We first recall the notion of seminormalisation of a scheme.

Definition 1. The seminormalisation $X^{s n} \rightarrow X$ of a scheme $X$ is an initial object in the full subcategory of schemes over $X$ consisting of universal homeomorphisms $Z \rightarrow X$ which induce isomorphisms on all residue fields.

By [34, Lemma 28.45.7], the seminormalisation always exists and $X^{s n}$ is a seminormal scheme, meaning that for any affine open $U$, the $\operatorname{ring} A=\mathcal{O}(U)$ is a seminormal ring -that is, if $x^{2}=y^{3}$ for some $x, y \in A$, then there is $a \in A$ with $x=a^{3}$ and $y=a^{2}$. Any seminormal scheme is reduced [34, Lemma 28.45.5], and hence we have a factorisation

$$
X^{s n} \rightarrow X^{r e d} \rightarrow X
$$

by the universal property of either the reduction or the seminormalisation.

By [34, Lemma 28.45.7], the seminormalisation is also the final object in the category of seminormal schemes above $X$ - that is,

$$
\operatorname{Hom}\left(Z, X^{s n}\right) \stackrel{\sim}{\longrightarrow} \operatorname{Hom}(Z, X)
$$

for any seminormal scheme $Z$. Any normal scheme is seminormal, and hence the normalisation $X^{n} \rightarrow X$ of, say, a Noetherian scheme $X$ [34, Section 28.52] factors through the seminormalisation $X^{n} \rightarrow X^{s n}$ by formula (20). If $X$ is, moreover, a Nagata scheme [34, Definition 27.13.1] - for example, if $X$ is of finite type over a field - then $X^{n} \rightarrow X$ is a finite morphism by [34, Lemma 28.52.10]. It follows that $X^{s n} \rightarrow X$ is finite, since $X$ is Noetherian.

Lemma 3. For any scheme $X$, the seminormalisation $X^{s n} \rightarrow X$ induces an equivalence of étale topoi

$$
X_{e t}^{s n} \cong X_{e t}
$$

For a scheme $X$ in $\mathrm{Sch}^{d} / \mathbb{F}_{p}$, the seminormalisation $X^{s n} \rightarrow X$ induces an isomorphism

$$
X^{s n, \sim} \cong X^{\sim}
$$

in $\left(\operatorname{Sch}^{d} / \mathbb{F}_{p}\right)_{\text {eh }}^{\sim}$, where $X^{\sim}$ denotes the eh-sheaf associated to the presheaf represented by $X$. Hence for any (abelian) eh-sheaf, $\mathcal{F}$ we have

$$
H^{i}\left(X_{e h}, \mathcal{F}\right) \cong H^{i}\left(X_{e h}^{s n}, \mathcal{F}\right)
$$

for all $i$.

Proof. Isomorphism (21) follows from the fact that $X^{s n} \rightarrow X$ is a universal homeomorphism (see [15, Exposé IX, 4.10] and [16, II, Exposé VIII, 1.1]). Since $X^{s n} \rightarrow X$ is finite surjective and induces an isomorphism on residue fields, it is an eh-cover by [9, Lemma 2.2]. Since it is also a monomorphism, it becomes an isomorphism in $\left(\operatorname{Sch}^{d} / \mathbb{F}_{p}\right)_{e h}^{\sim}$. 
Lemma 4. For schemes $X, Y$ in $\operatorname{Sch}^{d} / \mathbb{F}_{p}$, we have

$$
Y^{\sim}(X) \cong \operatorname{Hom}_{\mathrm{Sch}^{d} / \mathbb{F}_{p}}\left(X^{s n}, Y\right) .
$$

Proof. Since $X^{s n, \sim} \cong X^{\sim}$, we have $Y^{\sim}(X)=Y^{\sim}\left(X^{s n}\right)$ and we can assume that $X$ is seminormal. We follow the arguments of [36, 3.2]. Since $X$ is reduced, any surjection $X^{\prime} \rightarrow X$ (for example, the disjoint union of the schemes in an eh-cover) is an epimorphism in the category of schemes. Hence the separated presheaf associated to $\operatorname{Hom}(-, Y)$ still has value $\operatorname{Hom}(X, Y)$ on seminormal $X$, and the map

$$
\operatorname{Hom}(X, Y) \rightarrow Y^{\sim}(X)
$$

is injective. Any element $f^{\sim}$ of the right-hand side is represented by a family of morphisms $f_{i}: U_{i} \rightarrow Y$ on some eh-cover $\left\{U_{i} \rightarrow X\right\}_{i \in I}$ with $\left.f_{i}\right|_{U_{i} \times{ }_{X} U_{j}}=\left.f_{j}\right|_{U_{i} \times{ }_{X} U_{j}}$. By [9, Proposition 2.3], every eh-cover has a refinement of the form

$$
\left\{U_{i} \rightarrow X^{\prime} \rightarrow X\right\}_{i \in I}
$$

where $\left\{U_{i} \rightarrow X^{\prime}\right\}_{i \in I}$ is an étale cover and $p: X^{\prime} \rightarrow X$ is proper, so that for each $x \in X$ there is $y \in p^{-1}(\{x\})$ so that $\kappa(x) \rightarrow p_{*} \kappa(y)$ is an isomorphism. Since $U_{i} \times_{X^{\prime}} U_{j} \rightarrow U_{i} \times_{X} U_{j}$, we have $\left.f_{i}\right|_{U_{i} \times X^{\prime} U_{j}}=\left.f_{j}\right|_{U_{i} \times{ }_{X^{\prime}} U_{j}}$ - that is, the $f_{i}$ glue to a morphism $f^{\prime}: X^{\prime} \rightarrow Y$. Consider the Stein factorisation $X^{\prime} \stackrel{p_{1}}{\rightarrow} X^{\prime \prime} \rightarrow X$ of $p$. Since $p_{1, *}\left(\mathcal{O}_{X^{\prime}}\right)=\mathcal{O}_{X^{\prime \prime}}$, the proof of [36, Lemma 3.2.7] shows that $f^{\prime}$ descends to a morphism $f^{\prime \prime}: X^{\prime \prime} \rightarrow Y$. The proof of [36, Theorem 3.2.9] produces a factorisation $X^{\prime \prime} \rightarrow X^{\prime \prime \prime} \stackrel{p_{0}}{\longrightarrow} X$ so that $f^{\prime \prime}$ descends to $f^{\prime \prime \prime}$ : $X^{\prime \prime \prime} \rightarrow Y$ and $p_{0}$ is a universal homeomorphism. Moreover, $\kappa\left(p_{0}^{-1}(x)\right)=\kappa(x)$ for each $x \in X$, since there is a point $y \in X^{\prime}$ with isomorphic residue field. Since $X$ is seminormal, $p_{0}$ is an isomorphism and we find that $f^{\sim}$ is represented by a morphism $f \in \operatorname{Hom}(X, Y)$.

Corollary 1. We have

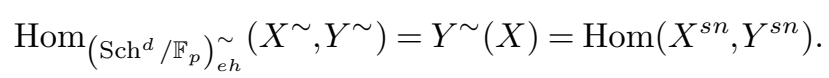

That is, the category of representable sheaves in the eh-topology is equivalent to the category of seminormal schemes.

Proof. This follows from Lemma 4 together with formula (20), or by applying Lemma 4 to both $Y^{s n}$ and $Y$ together with $Y^{s n, \sim} \cong Y^{\sim}$.

Recall the adjunction $\left(\rho_{d}^{*}, \rho_{d, *}\right)$ from formula (8).

Lemma 5. Let $\mathcal{F}$ be a sheaf on $\left(\mathrm{Sm}^{d} / \mathbb{F}_{p}\right)_{\text {et }}$ representable by a scheme $Y$. Under $R\left(d, \mathbb{F}_{p}\right)$, we have $\rho_{d}^{*} \mathcal{F}=Y^{\sim}$ and therefore

$$
\rho_{d}^{*} \mathcal{F}(X)=\operatorname{Hom}\left(X^{s n}, Y\right)
$$

for any $X$. 
Proof. Since every scheme has a cover by smooth schemes in the eh-topology under $R\left(d, \mathbb{F}_{p}\right)$, it suffices to show that $\rho_{d}^{*} \mathcal{F}(X)=Y^{\sim}(X)$ for smooth $X$. Clearly $\rho_{d}^{p} \mathcal{F}(X)=$ $\operatorname{Hom}(X, Y)$, where $\rho_{d}^{p}$ is the presheaf pullback. By [9, Corollary 2.6], every eh-cover of a smooth scheme has a refinement by a cover consisting of smooth schemes. Hence the eh-sheafification process again leads to isomorphic groups on both sides.

Next we discuss comparison results between étale and eh-cohomology. Consider the morphism of topoi $p=p_{X}:\left(\operatorname{Sch}^{d} / \mathbb{F}_{p}\right)_{e h}^{\sim} / X \rightarrow X_{e t}$ and the natural transformation

$$
\alpha^{\prime}:\left.\mathcal{F}\right|_{X} \rightarrow R p_{*} \mathcal{F}
$$

where $\left.\mathcal{F}\right|_{X}:=p_{*} \mathcal{F}$ denotes restriction to the small étale site. The functor $\left.\mathcal{F} \mapsto \mathcal{F}\right|_{X}$ extends to complexes but does not preserve quasi-isomorphisms. We obtain a natural transformation

$$
\alpha: R \Gamma\left(X_{e t},\left.\mathcal{F}\right|_{X}\right) \rightarrow R \Gamma\left(X_{e h}, \mathcal{F}\right)
$$

on the category of abelian sheaves on $\left(\operatorname{Sch}^{d} / \mathbb{F}_{p}\right)_{e h}$. Both $\alpha^{\prime}$ and $\alpha$ are also contravariantly functorial in $X$.

Lemma 6. Assume $\mathcal{F}$ is a torsion sheaf in $\mathrm{Ab}\left(\mathrm{Sch}^{d} / \mathbb{F}_{p}\right)_{\text {eh }}^{\sim}$. Then there exists a natural transformation

$$
\alpha_{c}: R \Gamma_{c}\left(X_{e t},\left.\mathcal{F}\right|_{X}\right) \rightarrow R \Gamma_{c}\left(X_{e h}, \mathcal{F}\right)
$$

which coincides with formula (23) for proper $X$ and is compatible with exact localisation triangles for open/closed decompositions $U \hookrightarrow X \hookleftarrow Z$.

Proof. Let $j: X \rightarrow \bar{X}$ be an open embedding into a proper $\mathbb{F}_{p}$-scheme $\bar{X}$ with closed complement $i: Z \hookrightarrow \bar{X}$. Choose an injective resolution $\mathcal{F} \rightarrow I^{\bullet}$ in $\operatorname{Ab}\left(\operatorname{Sch}^{d} / \mathbb{F}_{p}\right)_{e h}^{\sim}$ and injective resolutions $\left.\mathcal{F}\right|_{\bar{X}} \rightarrow J_{\bar{X}}^{\bullet}$ (resp., $\left.i^{*}\left(\left.\mathcal{F}\right|_{\bar{X}}\right) \rightarrow J_{Z}^{\bullet}\right)$ in $\bar{X}_{e t}$ (resp., $\left.Z_{e t}\right)$. Then the commutative diagram on $\bar{X}_{e t}$

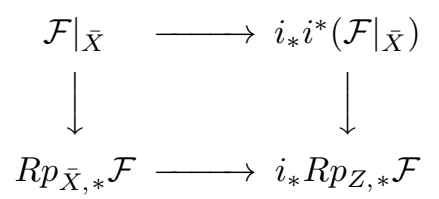

which arises from the functoriality of $\alpha^{\prime}$ for the morphism $i$ is realised by a diagram of maps of complexes of injectives

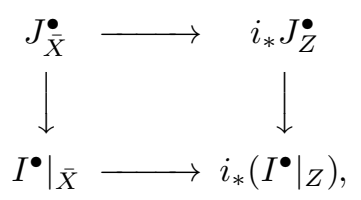

with all maps unique up to homotopy and commuting up to homotopy. Note here that $p_{*}$ and $i_{*}$ preserve injective objects. Taking global sections on $\bar{X}_{e t}$ and taking mapping fibres of the horizontal maps gives $\alpha_{c}$ unique up to homotopy. For $R \Gamma_{c}\left(X_{e h}, \mathcal{F}\right)$ this is 
[9, Definition 3.3], and for $R \Gamma_{c}\left(X_{e t},\left.\mathcal{F}\right|_{X}\right)$ this is the usual definition of compact support cohomology on the small étale site, since $\left.\mathcal{F}\right|_{X}=\left.j^{*} \mathcal{F}\right|_{\bar{X}}$. As $\left.\mathcal{F}\right|_{X}$ is torsion, this definition is independent of the choice of $j$, and so is $R \Gamma_{c}\left(X_{e h}, \mathcal{F}\right)$, by [9, Lemma 3.4]. The functoriality for open/closed decompositions follows by applications of the octahedral axiom.

Corollary 2. Let $\mathcal{F} \in \mathrm{Ab}\left(\operatorname{Sch}^{d} / \mathbb{F}_{p}\right)_{\text {eh }}^{\sim}$ be a torsion sheaf such that

$$
\alpha: R \Gamma\left(X_{e t},\left.\mathcal{F}\right|_{X}\right) \cong R \Gamma\left(X_{e h}, \mathcal{F}\right)
$$

is an isomorphism for smooth, proper $X$. If we assume $R\left(d, \mathbb{F}_{p}\right)$, then

$$
\alpha_{c}: R \Gamma_{c}\left(X_{e t},\left.\mathcal{F}\right|_{X}\right) \rightarrow R \Gamma_{c}\left(X_{e h}, \mathcal{F}\right)
$$

is an isomorphism for all $X$.

Proof. The proof is a standard induction over the dimension of $X$ (see [9, Lemma $2.7])$.

We remark that

$$
\alpha: R \Gamma\left(X_{e t},\left.\mathcal{F}\right|_{X}\right) \cong R \Gamma\left(X_{e h}, \mathcal{F}\right)
$$

is an isomorphism for all $X$ if $\left.\mathcal{F}\right|_{\mathrm{Ab}\left(\operatorname{Sch}^{d} / \mathbb{F}_{p}\right)_{e t}^{\sim}}$ is constructible, without further assumptions, by [9, Theorem 3.6]. The transformation $\alpha_{c}$ is then also an isomorphism for all $X$ if $\mathcal{F}$ is constructible. However, constructibility is a much stronger assumption than being torsion, and will not hold for the $p$-primary torsion sheaves of interest later.

For nontorsion sheaves, even if étale and eh-cohomology agree on smooth schemes $X$, one cannot expect an isomorphism for general, even normal schemes, as [9, Proposition 8.2] shows. The following lemma (which is not needed in the remainder of the paper) allows us to prove such an identification between étale and eh-cohomology in some very restricted circumstances:

Lemma 7. Let $\mathcal{F} \in \mathrm{Ab}\left(\operatorname{Sch}^{d} / \mathbb{F}_{p}\right)_{\text {eh }}^{\sim}$ be such that

$$
\alpha: R \Gamma\left(X_{e t},\left.\mathcal{F}\right|_{X}\right) \cong R \Gamma\left(X_{e h}, \mathcal{F}\right)
$$

is an isomorphism for smooth $X$. Let

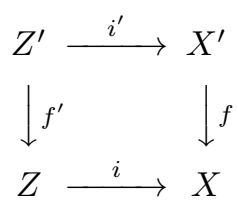

be an abstract blowup square [9, Definition 2.1] with $f$ finite and $Z, Z^{\prime}$ and $X^{\prime}$ smooth. Assume $\left.\left.\left.f_{*} \mathcal{F}\right|_{X^{\prime}} \oplus i_{*} \mathcal{F}\right|_{Z} \rightarrow g_{*} \mathcal{F}\right|_{Z^{\prime}}$ is surjective where $g=i f^{\prime}$. Then

$$
\alpha: R \Gamma\left(X_{e t},\left.\mathcal{F}\right|_{X}\right) \cong R \Gamma\left(X_{e h}, \mathcal{F}\right)
$$

is an isomorphism. 
Proof. The functoriality of $p$ in $X$ and diagram (27) induce a commutative diagram of exact triangles

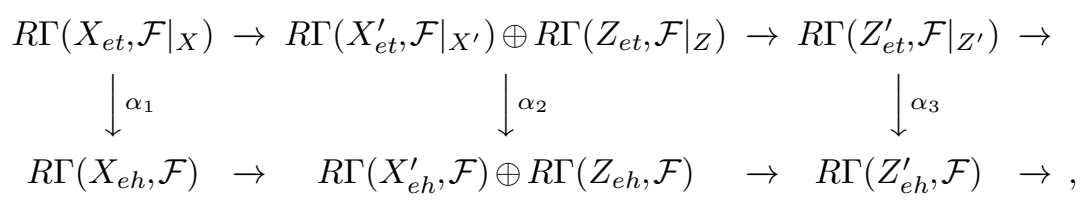

where the bottom row is exact by [9, Proposition 3.2] and the top row is induced by the exact sequence of sheaves on $X_{e t}$

$$
\left.\left.\left.\left.0 \rightarrow \mathcal{F}\right|_{X} \rightarrow f_{*} \mathcal{F}\right|_{X^{\prime}} \oplus i_{*} \mathcal{F}\right|_{Z} \rightarrow g_{*} \mathcal{F}\right|_{Z^{\prime}} \rightarrow 0 .
$$

Here exactness at the last term was assumed, and exactness at the remaining terms follows from the fact that $\mathcal{F}$ is an eh-sheaf, and the pullback of diagram (27) to any étale $U \rightarrow X$ is again an abstract blowup square. We also use that $f, i, g$ are finite so that $R \Gamma\left(X_{e t},\left.f_{*} \mathcal{F}\right|_{X^{\prime}}\right)=R \Gamma\left(X_{e t}^{\prime},\left.\mathcal{F}\right|_{X^{\prime}}\right)$, and so on. Since $Z^{\prime}, Z, X^{\prime}$ are smooth, the maps $\alpha_{2}, \alpha_{3}$ are quasi-isomorphisms, and hence so is $\alpha_{1}$ by the five lemma.

Corollary 3. Let $X$ be a seminormal curve over $\mathbb{F}_{p}$. Then we have isomorphisms

$$
R \Gamma\left(X_{e t}, \mathbb{G}_{m}\right) \cong R \Gamma\left(X_{e h}, \rho_{d}^{*} \mathbb{G}_{m}\right)
$$

and

$$
R \Gamma\left(X_{e t}, \mathcal{O}\right) \cong R \Gamma\left(X_{e h}, \rho_{d}^{*} \mathcal{O}\right)
$$

Proof. Since $R\left(1, \mathbb{F}_{p}\right)$ holds, we have $\rho_{d}^{*} \mathbb{G}_{m}=\mathbb{G}_{m}^{\sim}$ by Lemma 5 , and since $X$ is seminormal, we have $\left.\mathbb{G}_{m}^{\sim}\right|_{X}=\mathbb{G}_{m}$ by Lemma 4 . Consider diagram (27) with $X^{\prime}=X^{n}$, the normalisation, and $Z=X^{\text {sing }}$, the singular locus. Both $Z$ and $Z^{\prime}$ are finite unions of closed points, hence smooth, and $X^{n}$ is smooth since $X$ is a curve. The map $\mathbb{G}_{m} \rightarrow i_{*}^{\prime} \mathbb{G}_{m}$ is surjective, since $i^{\prime}$ is a closed embedding, and applying $f_{*}$ gives a surjection, since $f_{*}$ is exact. So all conditions of Lemma 7 are satisfied. The proof for $\mathcal{O}$ is identical, since $\mathcal{O}$ is representable by $\mathbb{A}^{1}$.

Corollary 4. Let $X$ be an arbitrary curve over $\mathbb{F}_{p}$. Then we have isomorphisms

$$
R \Gamma\left(X_{e h}, \rho_{d}^{*} \mathbb{G}_{m}\right) \cong R \Gamma\left(X_{e t}^{s n}, \mathbb{G}_{m}\right)
$$

and

$$
R \Gamma\left(X_{e h}, \rho_{d}^{*} \mathcal{O}\right) \cong R \Gamma\left(X_{e t}^{s n}, \mathcal{O}\right)
$$

Proof. Combine formula (22) and Corollary 3.

We finally come back to the proof of Proposition 2, and shall assume $R\left(d, \mathbb{F}_{p}\right)$. For $X$ in $\mathrm{Sch}^{d} / \mathbb{F}_{p}$, we have a natural map

$$
R \Gamma\left(X_{e t}, \mathbb{G}_{m}\right) \rightarrow R \Gamma\left(X_{e t},\left.\mathbb{G}_{m}^{\sim}\right|_{X}\right) \stackrel{\alpha}{\rightarrow} R \Gamma\left(X_{e h}, \rho_{d}^{*} \mathbb{G}_{m}\right)
$$


by Lemma 5 . Consider the induced map on $p$-adic completions and denote by $C$ the [-1]-shift of its mapping cone, so that there is an exact triangle

$$
R \Gamma\left(X_{e t}, \mathbb{Z}_{p}(1)\right) \rightarrow R \Gamma\left(X_{e h}, \mathbb{Z}_{p}(1)\right) \rightarrow C .
$$

Similarly, we have an exact triangle

$$
R \Gamma\left(X_{e t}, \mathcal{O}\right)[-1] \rightarrow R \Gamma\left(X_{e h}, \rho_{d}^{*} \mathcal{O}\right)[-1] \rightarrow C^{a d d} .
$$

For a bounded complex $K$ with finite cohomology, we set

$$
\chi(K):=\sum_{j}(-1)^{j} \operatorname{ord}_{p} \# H^{j}(K) .
$$

For example, if $X$ is proper, the terms in triangle (29) are perfect complexes of $\mathbb{F}_{p}$-vector spaces by [9, Corollary 4.8], and we have

$$
\chi\left(C^{a d d}\right)=\chi_{e t}(X, \mathcal{O})-\chi(X, \mathcal{O}),
$$

where $\chi(X, \mathcal{O})$ (resp., $\left.\chi_{e t}(X, \mathcal{O})\right)$ is defined as in definition (11) (resp., definition (13)), with $\mathcal{X}_{\mathbb{F}_{p}}$ replaced by $X$.

Proposition 2.1. Let $X \rightarrow \operatorname{Spec}\left(\mathbb{F}_{p}\right)$ be proper of dimension $d$ and assume $R\left(d, \mathbb{F}_{p}\right)$. Then $C$ is a bounded complex with finite cohomology and

$$
\chi(C)=\chi\left(C^{a d d}\right) .
$$

In particular, for a proper arithmetic scheme $\mathcal{X}$ we have an isomorphism

$$
R \Gamma\left(\mathcal{X}_{\mathbb{F}_{p}, e t}, \mathbb{Q}_{p}(1)\right) \cong R \Gamma\left(\mathcal{X}_{\mathbb{F}_{p}, e h}, \mathbb{Q}_{p}(1)\right)
$$

so that

$$
\operatorname{det}_{\mathbb{Z}_{p}} R \Gamma\left(\mathcal{X}_{\mathbb{F}_{p}, e t}, \mathbb{Z}_{p}(1)\right)=p^{\chi e t}\left(\mathcal{X}_{\mathbb{F}_{p}}, \mathcal{O}\right)-\chi\left(\mathcal{X}_{\mathbb{F}_{p}}, \mathcal{O}\right) \cdot \operatorname{det}_{\mathbb{Z}_{p}} R \Gamma\left(\mathcal{X}_{\mathbb{F}_{p}, e h}, \mathbb{Z}_{p}(1)\right)
$$

inside $\operatorname{det}_{\mathbb{Q}_{p}} R \Gamma\left(\mathcal{X}_{\mathbb{F}_{p}, e h}, \mathbb{Q}_{p}(1)\right)$.

Proof. First recall that $\mathbb{G}_{m}^{\sim} \cong \rho_{d}^{*} \mathbb{G}_{m}$, by Lemma 5. Since $\left.\mathbb{G}_{m}^{\sim}\right|_{Z}=\mathbb{G}_{m}$ for smooth $Z$, the assumption of Corollary 2 is satisfied for $\mathbb{G}_{m} / p^{\nu}$ by [9, Theorem 4.3], and we deduce that the $p$-adic completion of $\alpha$ in map (28) is an isomorphism for proper $X$. Moreover, by Lemma 4 we have $\left.\mathbb{G}_{m}^{\sim}\right|_{X}=\sigma_{*} \mathbb{G}_{m}$, where $\sigma: X^{s n} \rightarrow X$ is the seminormalisation. So we obtain an exact triangle

$$
R \Gamma\left(X_{e t}, \mathbb{Z}_{p}(1)\right) \rightarrow R \Gamma\left(X_{e t}^{s n}, \mathbb{Z}_{p}(1)\right) \rightarrow C .
$$

Since $\mathcal{O}$ is a $p$-torsion sheaf, an analogous argument gives an exact triangle

$$
R \Gamma\left(X_{e t}, \mathcal{O}\right)[-1] \rightarrow R \Gamma\left(X_{e t}^{s n}, \mathcal{O}\right)[-1] \rightarrow C^{a d d} .
$$

We have an exact sequence of coherent sheaves on $X_{e t} \cong X_{e t}^{s n}$

$$
0 \rightarrow J \rightarrow \mathcal{O}_{X} \rightarrow \sigma_{*} \mathcal{O}_{X^{s n}} \rightarrow K \rightarrow 0,
$$


where $J$ is the nilradical, as $X^{s n}$ is reduced. There is an analogous sequence of abelian sheaves

$$
1 \rightarrow 1+J \rightarrow \mathbb{G}_{m, X} \rightarrow \sigma_{*} \mathbb{G}_{m, X^{s n}} \rightarrow K^{m u l t} \rightarrow 1 .
$$

Both $J$ and $1+J$ have finite filtrations $J^{k}$ and $1+J^{k}$, respectively, with isomorphic subquotients

$$
J^{k} / J^{k+1} \cong\left(1+J^{k}\right) /\left(1+J^{k+1}\right), \quad x \mapsto 1+x,
$$

and hence

$$
\chi\left(R \Gamma\left(X_{e t}, J\right)\right)=\chi\left(R \Gamma\left(X_{e t}, 1+J\right)\right) .
$$

To prove equation (30), it then suffices to show $\chi\left(R \Gamma\left(X_{e t}, K\right)\right)=\chi\left(R \Gamma\left(X_{e t}, K^{m u l t}\right)\right)$. This in turn will follow from the more general statement that

$$
\chi\left(R \Gamma\left(X_{e t}, \sigma_{*}^{\prime} \mathcal{O}_{X^{\prime}} / \mathcal{O}_{X}\right)\right)=\chi\left(R \Gamma\left(X_{e t}, \sigma_{*}^{\prime} \mathbb{G}_{m, X^{\prime}} / \mathbb{G}_{m, X}\right)\right)
$$

for any universal homeomorphism

$$
\sigma^{\prime}: X^{\prime} \rightarrow X
$$

inducing isomorphisms on all residue fields with $X$ reduced. We prove this by induction on the dimension $d$ of $X$. First we can assume that $X^{\prime}$ is reduced, using equation (31) for the nilradical of $X^{\prime}$. If $d=0$, both $X$ and $X^{\prime}$ are finite unions of spectra of finite fields and $\sigma^{\prime}$ is an isomorphism. In general, let $Z \hookrightarrow X$ be the singular locus, a proper closed subset of $X$, with its reduced scheme structure, and let

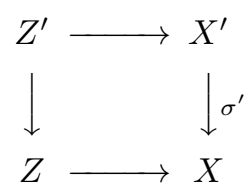

be the pullback under $\sigma^{\prime}$. Then $Z^{\prime} \rightarrow Z$ is again a universal homeomorphism inducing isomorphisms on all residue fields with $Z$ reduced, to which the induction assumption applies.

Since $X \backslash Z$ is smooth, hence seminormal, the restriction of $\sigma^{\prime}$ to $X^{\prime} \backslash Z^{\prime}$ has a section, and hence is an isomorphism, as $X^{\prime}$ is reduced. It follows that $\mathcal{O}_{X^{\prime}} / \mathcal{O}_{X}$ is supported on $Z$ (we omit $\sigma_{*}^{\prime}$, since $\sigma^{\prime}$ is a homeomorphism). The morphism $\sigma^{\prime}$ is finite, as the seminormalisation factors through it. Hence $\mathcal{O}_{X^{\prime}} / \mathcal{O}_{X}$ is coherent and there exists $r$ such that $\mathcal{M}^{r} \cdot \mathcal{O}_{X^{\prime}} / \mathcal{O}_{X}=0$, where $\mathcal{M}$ denotes the ideal sheaf of $Z$. Setting $\mathcal{M}^{\prime}=\mathcal{M} \mathcal{O}_{X^{\prime}}$, we have an exact sequence of coherent sheaves

$$
0 \rightarrow \mathcal{M}^{\prime} / \mathcal{M} \rightarrow \mathcal{O}_{X^{\prime}} / \mathcal{O}_{X} \rightarrow \mathcal{O}_{Z^{\prime}} / \mathcal{O}_{Z} \rightarrow 0
$$

supported on $Z$. Since $\left(\mathcal{M}^{\prime}\right)^{r+1}=\mathcal{M}^{r+1} \mathcal{O}_{X^{\prime}} \subseteq \mathcal{M}$, we have a finite filtration by coherent subsheaves

$$
\mathcal{M}^{\prime} / \mathcal{M} \supseteq \cdots \supseteq \mathcal{N}^{i} \supseteq \cdots \supseteq \mathcal{N}^{r+1}=0
$$


where $\mathcal{N}^{i}:=\left(\mathcal{M}^{\prime}\right)^{i} /\left(\mathcal{M}^{\prime}\right)^{i} \cap \mathcal{M}$. On the multiplicative side, we have an exact sequence of abelian sheaves on $X_{e t}$ supported in $Z$

$$
0 \rightarrow\left(1+\mathcal{M}^{\prime}\right) /(1+\mathcal{M}) \rightarrow \mathbb{G}_{m, X^{\prime}} / \mathbb{G}_{m, X} \rightarrow \mathbb{G}_{m, Z^{\prime}} / \mathbb{G}_{m, Z} \rightarrow 1
$$

with corresponding filtration

$$
\left(1+\mathcal{M}^{\prime}\right) /(1+\mathcal{M})=1+\mathcal{M}^{\prime} / \mathcal{M} \supseteq \cdots \supseteq 1+\mathcal{N}^{i} \supseteq \cdots \supseteq 1+\mathcal{N}^{r+1}=1
$$

and an isomorphism of subquotients

$$
\mathcal{N}^{i} / \mathcal{N}^{i+1} \cong\left(1+\mathcal{N}^{i}\right) /\left(1+\mathcal{N}^{i+1}\right), \quad x \mapsto 1+x .
$$

Note here that all sections $1+x \in 1+\mathcal{M}^{\prime} / \mathcal{M}$ are invertible, since $x^{r+1} \in \mathcal{M}$. We conclude that

$$
\chi\left(R \Gamma\left(X_{e t}, \mathcal{M}^{\prime} / \mathcal{M}\right)\right)=\chi\left(R \Gamma\left(X_{e t}, 1+\mathcal{M}^{\prime} / \mathcal{M}\right)\right),
$$

and together with the induction assumption we obtain equation (32).

\section{Artin-Verdier duality}

A key ingredient in our construction of Weil-étale complexes is Artin-Verdier duality with torsion coefficients, in the form of Conjecture $\mathbf{A V}(\mathcal{X}, n)$ introduced in [6, Conjecture 6.23]. The compact support cohomology $\hat{H}_{c}^{i}$ is defined as in [27, II, 2.3] using Tate cohomology at all Archimedean places.

Conjecture $\mathbf{3}(\mathbf{A V}(\mathcal{X}, n))$. There is a symmetric product map

$$
\mathbb{Z}(n) \otimes^{L} \mathbb{Z}(d-n) \rightarrow \mathbb{Z}(d)
$$

in $\mathcal{D}\left(\mathcal{X}_{e t}\right)$ such that the induced pairing

$$
\widehat{H}_{c}^{i}\left(\mathcal{X}_{e t}, \mathbb{Z} / m(n)\right) \times H^{2 d+1-i}\left(\mathcal{X}_{e t}, \mathbb{Z} / m(d-n)\right) \rightarrow \widehat{H}_{c}^{2 d+1}\left(\mathcal{X}_{e t}, \mathbb{Z} / m(d)\right) \rightarrow \mathbb{Q} / \mathbb{Z}
$$

is a perfect pairing of finite abelian groups for any $i \in \mathbb{Z}$ and any positive integer $m$.

This conjecture is known for $\mathcal{X}$ smooth proper over a number ring, and for regular proper $\mathcal{X}$ as long as $n \leq 0$ or $n \geq d$. Therefore, if $\mathcal{X}$ is an arithmetic surface, the only unresolved case is $n=1$, which we shall prove using results of Saito [30].

Proposition 3.1. Conjecture $\mathbf{A V}(\mathcal{X}, 1)$ holds if $\mathcal{X}$ is an arithmetic surface.

Proof. It suffices to prove the statement for an arbitrary prime power $m=p^{\nu}$. Consider the open/closed decomposition

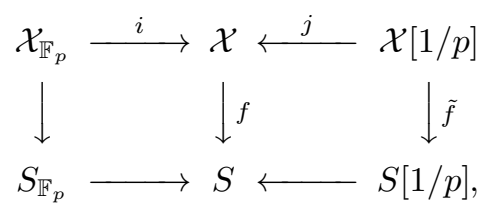


and note that $j^{*} \mathbb{Z}(1) / p^{\nu} \cong \mu_{p^{\nu}}$. Denoting by $A^{*}:=R \operatorname{Hom}(A, \mathbb{Q} / \mathbb{Z})$ the $\mathbb{Q} / \mathbb{Z}$-dual, we have isomorphisms

$$
\begin{aligned}
R \Gamma\left(\mathcal{X}[1 / p], \mu_{p^{\nu}}\right) & \cong R \Gamma\left(\mathcal{X}[1 / p], R \underline{\operatorname{Hom}}_{\mathcal{X}[1 / p]}\left(\mu_{p^{\nu}}, \mu_{p^{\nu}}^{\otimes 2}\right)\right) \\
& \cong R \operatorname{Hom}_{\mathcal{X}[1 / p]}\left(\mu_{p^{\nu}}, \mu_{p^{\nu}}^{\otimes 2}\right) \\
& \cong R \operatorname{Hom}_{\mathcal{X}[1 / p]}\left(\mu_{p^{\nu}}, R \tilde{f}^{!} \mu_{p^{\nu}}\right)[-2] \\
& \cong R \operatorname{Hom}_{S[1 / p]}\left(R \tilde{f}_{*} \mu_{p^{\nu}}, \mu_{p^{\nu}}\right)[-2] \\
& \cong \hat{R} \Gamma_{c}\left(S[1 / p], R \tilde{f}_{*} \mu_{p^{\nu}}\right)^{*}[-5] \\
& \cong \hat{R} \Gamma_{c}\left(\mathcal{X}[1 / p], \mu_{p^{\nu}}\right)^{*}[-5]
\end{aligned}
$$

using purity $\tilde{f}^{!} \mu_{p^{\nu}} \cong \mu_{p^{\nu}}^{\otimes 2}[2]$, as $\mathcal{X}$ and $S$ are regular of dimensions 2 and 1 , respectively [8]; the adjunction $\left(R \tilde{f}_{*}, \tilde{f}^{!}\right)$, since $\tilde{f}$ is proper; and Artin-Verdier duality on $S[1 / p][27$, II, Theorem 3.1]. Also note that $R \tilde{f}_{*} \mu_{p^{\nu}}$ is a complex with constructible cohomology, as $\tilde{f}$ is proper.

Let $\mathfrak{X}$ be the formal completion of $\mathcal{X}$ at the ideal sheaf $I=(p)$, where we view the structure sheaf $\mathcal{O}_{\mathfrak{X}}$ as a sheaf on $\mathcal{X}_{\mathbb{F}_{p}, \text { et }}$. By [30, Theorem 4.13], the map

$$
H_{\mathcal{X}_{\mathbb{F}_{p}}}^{4-i}\left(\mathcal{X}, \mathbb{G}_{m}\right) \rightarrow H^{i}\left(\mathcal{X}_{\mathbb{F}_{p}}, \mathbb{G}_{m, \mathfrak{X}}\right)^{*}
$$

is an isomorphism for $i=0,1$. The groups $H^{i}\left(\mathcal{X}_{\mathbb{F}_{p}}, \mathbb{G}_{m, \mathfrak{X}}\right)$ can be computed following the computation of $H^{i}\left(\mathcal{X}_{\mathbb{F}_{p}}, i^{*} \mathbb{G}_{m}\right)$ in [30, Proposition 4.6(2)], and the result is the same for $i \neq 0,1$. More precisely, the rings $\mathcal{O}_{x}, \mathcal{O}_{\eta}, \mathcal{O}_{\lambda}$ of that proposition are replaced by the corresponding local rings of $\mathfrak{X}$, which are again Henselian local with unchanged residue field. Since $\mathbb{G}_{m}$ is a smooth group scheme, its cohomology in degrees $\geq 1$ coincides with that of the residue field [26, III.3.11].

It follows then from [30, Proposition 4.6(1) and (2)] that map (35) is an isomorphism for $i \neq 3$ and has as cokernel the uniquely divisible group $(\hat{\mathbb{Z}} / \mathbb{Z})^{r}$ for $i=3$, where $r$ is the number of irreducible components of $\mathcal{X}_{\mathbb{F}_{p}}$. Hence

$$
H_{\mathcal{X}_{p}}^{3-i}\left(\mathcal{X}, \mathbb{G}_{m} / p^{\nu}\right) \rightarrow H^{i}\left(\mathcal{X}_{\mathbb{F}_{p}}, \mathbb{G}_{m, \mathfrak{X}} / p^{\nu}\right)^{*}
$$

is an isomorphism for all $i$. On the other hand, by Lemma 2 we have an isomorphism $i^{*} \mathbb{G}_{m} / p^{\nu} \cong \mathbb{G}_{m, \mathfrak{x}} / p^{\nu}$ and hence an isomorphism

$$
H_{\mathcal{X}_{\mathbb{F}_{p}}}^{3-i}\left(\mathcal{X}, \mathbb{G}_{m} / p^{\nu}\right) \stackrel{\sim}{\rightarrow} H^{i}\left(\mathcal{X}_{\mathbb{F}_{p}}, i^{*} \mathbb{G}_{m} / p^{\nu}\right)^{*}
$$

or equivalently

$$
H_{\mathcal{X}_{\mathbb{F}_{p}}}^{5-i}\left(\mathcal{X}, \mathbb{Z}(1) / p^{\nu}\right) \stackrel{\sim}{\longrightarrow} H^{i}\left(\mathcal{X}_{\mathbb{F}_{p}}, i^{*} \mathbb{Z}(1) / p^{\nu}\right)^{*}
$$


for all $i$. Now consider the duality map on localisation triangles

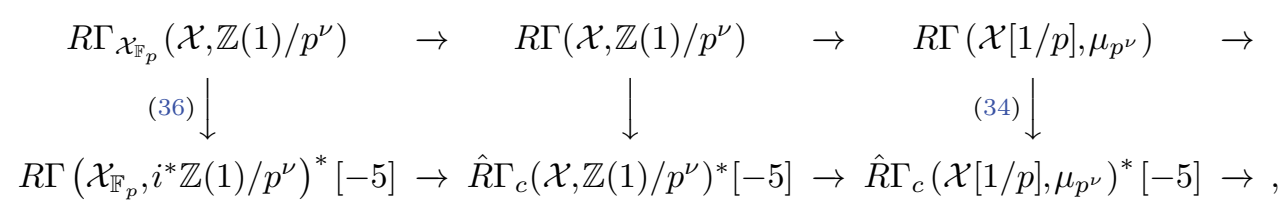

where we have shown the outer vertical maps to be quasi-isomorphisms. It follows that the middle vertical map is a quasi-isomorphism, which finishes the proof.

The remainder of this section is aimed at the proof of Corollary 5. The results of Saito involve Lichtenbaum's pairing [30, (1.1)]

$$
\mathbb{G}_{m}[-1] \otimes^{L} \mathbb{G}_{m}[-1] \rightarrow \mathbb{Z}(2)^{L i}
$$

together with the trace map

$$
H^{6}\left(\mathcal{X}, \mathbb{Z}(2)^{L i}\right) \rightarrow \mathbb{Q} / \mathbb{Z}
$$

constructed in [30, Theorem 3.1]. The relation to Bloch's complex $\mathbb{Z}(2)$ is given by maps

$$
\mathbb{Z}(2)^{L i} \rightarrow \mathcal{K}_{/ \mathcal{X}} \stackrel{\sim}{\leftarrow} \tau^{\geq 1} \mathbb{Z}(2) \leftarrow \mathbb{Z}(2),
$$

where $\mathcal{K}_{/ \mathcal{X}}$ is the complex constructed by Spiess in [33] and the middle isomorphism is [38, Theorem 3.8]. Pairing (37), combined with map (38) and taken modulo $p^{\nu}$, can also be characterised as the unique pairing constructed by the method of Sato in [31]. Even though Sato assumes $\mathcal{X}$ to have semistable reduction, his arguments work in our situation where $\mathcal{X}$ is a relative curve and $n=m=1$. We summarise the properties we need in the following proposition:

\section{Proposition 3.2.}

(a) The map $\left(\tau^{\geq 1} \mathbb{Z}(2)\right) / p^{\nu} \leftarrow \mathbb{Z}(2) / p^{\nu}$ is a quasi-isomorphism.

(b) The pairing

$$
\mathbb{Z}(1) / p^{\nu} \otimes^{L} \mathbb{Z}(1) / p^{\nu} \rightarrow \mathbb{Z}(2) / p^{\nu}
$$

obtained by combining formulas (37) and (38) and (a) is the unique pairing extending

$$
\mu_{p^{\nu}} \otimes \mu_{p^{\nu}} \cong \mu_{p^{\nu}}^{\otimes 2}
$$

on $\mathcal{X}[1 / p]_{\text {et }}$.

(c) There is a commutative diagram in $D\left(S_{e t}, \mathbb{Z} / p^{\nu}\right)$

$$
\begin{gathered}
R f_{*} \mathbb{Z}(1) / p^{\nu}[2] \otimes^{L} \mathbb{Z}(1) / p^{\nu} \quad \stackrel{\operatorname{Tr}_{f}^{0} \otimes \mathrm{id}}{\longrightarrow} \mathbb{Z}(0) / p^{\nu} \otimes^{L} \mathbb{Z}(1) / p^{\nu} \\
\operatorname{id} \otimes f^{*} \downarrow \\
R f_{*} \mathbb{Z}(1) / p^{\nu}[2] \otimes^{L} R f_{*} \mathbb{Z}(1) / p^{\nu} \\
\longrightarrow
\end{gathered}
$$


where $\operatorname{Tr}_{f}$ (resp., $f^{*}$ ) is constructed as in [31, Theorem 7.1.1] (resp., [31, Proposition 4.2.81).

Proof. Since $\mathcal{K}_{/ \mathcal{X}}$ is concentrated in degrees 1 and 2 by [33, 1.6.2.(A1)], it follows that $\mathbb{Z}(2)$ is concentrated in degrees $\leq 2-$ that is, [6, Conjecture 7.1] holds true. By [6, Lemma 7.7], there is then an exact triangle

$$
\tau^{\leq 1}\left(i_{*} \mathbb{Z}(1)^{Z} / p^{\nu}\right)[-2] \rightarrow \mathbb{Z}(2) / p^{\nu} \rightarrow \tau^{\leq 2} R j_{*} \mu_{p^{\nu}}^{\otimes 2} \rightarrow
$$

where $i$ and $j$ are as in diagram (33) and we set $Z:=\mathcal{X}_{\mathbb{F}_{p}}$. Hence $\mathbb{Z}(2) / p^{\nu}$ is concentrated in degrees $0,1,2$ and $\mathcal{H}^{0}\left(\mathbb{Z}(2) / p^{\nu}\right) \cong j_{*} \mu_{p^{\nu}}^{\otimes 2} \cong j_{*} \mathcal{H}^{1}(\mathbb{Z}(2))_{p^{\nu}}$. This last identity follows from

$$
\left(\mathcal{H}^{1} \mathcal{K}_{/ \mathcal{X}}\right)_{p^{\nu}} \cong \eta_{*} \mathcal{H}^{1}\left(\mathbb{Z}(2)^{L i}\right)_{p^{\nu}} \cong \eta_{*} K_{3}^{\text {ind }}(\bar{L})_{p^{\nu}} \cong \eta_{*} \mu_{p^{\nu}}^{\otimes 2}
$$

where $\eta: \operatorname{Spec}(L) \rightarrow \mathcal{X}$ is the function field of $\mathcal{X}$ (see the proof of [33, 1.5.1]). This concludes the proof of (a).

By [38, Theorem 1.1], the complex $\mathbb{Z}(1)^{Z} / p^{\nu}$ is quasi-isomorphic to the logarithmic de Rham-Witt complex

$$
\begin{aligned}
\mathbb{Z}(1)^{Z} / p^{\nu} & \cong\left[\bigoplus_{z \in Z^{0}} i_{z, *} W_{\nu} \Omega_{\kappa(z), \log }^{1} \stackrel{\partial}{\rightarrow} \bigoplus_{x \in Z^{1}} i_{x, *} W_{\nu} \Omega_{\kappa(x), \log }^{0}\right] \\
& \cong\left[\bigoplus_{z \in Z^{0}} i_{z, *}\left(\kappa(z)^{\times}\right) / p^{\nu} \stackrel{\partial}{\rightarrow} \bigoplus_{x \in Z^{1}} i_{x, *} \mathbb{Z} / p^{\nu}\right]
\end{aligned}
$$

placed in degrees 1 and 2. Note, incidentally, that this complex depends only on the underlying topological space and the residue fields of $Z$, and hence coincides for $Z$ and $Z^{s n}$. The stalk of $\partial$ at a point $x \in Z^{1}$ is the map

$$
\bigoplus_{x \in \overline{\{z\}}}\left(\kappa(z)^{\times}\right) / p^{\nu} \stackrel{\sum_{z, P} v_{P}}{\longrightarrow} \mathbb{Z} / p^{\nu},
$$

where for each irreducible component $\overline{\{z\}}$ of $Z$ passing through $x$, the sum is over all valuations of $\kappa(z)$ lying over $x$ - that is, over all points $P$ of the normalisation of $\overline{\{z\}}$ lying above $x$. Since we are considering stalks in the étale topology, we can assume the base field is $\overline{\mathbb{F}}_{p}$, hence infinite. Let $U \subseteq \mathbb{A}^{N}$ be an affine neighbourhood of the points $P$. Then there exists a hyperplane $H \subseteq \mathbb{A}^{N}$ intersecting $U$ transversely in one of the $P$, say $P_{0}$, and not in any other $P$. The linear form with zero set $H$, restricted to $U$, gives a function $f \in \kappa(z)$ with $v_{P_{0}}(f)=1$ and $v_{P}(f)=0$ for $P \neq P_{0}$ - that is, $\partial_{x}(f)=1$. Hence $\partial$ is surjective and

$$
\mathbb{Z}(1)^{Z} / p^{\nu} \cong \operatorname{ker}(\partial)[-1]
$$

is concentrated in degree 1 . In the notation of $[31,2.2]$, we have $\operatorname{ker}(\partial) \cong \nu_{Z, \nu}^{1}$. The localisation triangle (41) then shows that $\mathbb{Z}(2) / p^{\nu}$ satisfies the defining properties of the 
complex $\mathfrak{T}_{\nu}(2)_{\mathcal{X}}$ constructed by Sato in [31, Lemma 4.2.2] (under the semistable reduction assumption). Similarly, the complex $\mathbb{Z}(1) / p^{\nu}=\mathbb{G}_{m}[-1] / p^{\nu}$ satisfies the defining properties of $\mathfrak{T}_{\nu}(1)_{\mathcal{X}}$, since the proof of [31, Proposition 4.5.1] in fact goes through for arbitrary regular $\mathcal{X}$.

The proof of [31, Proposition 4.2.6] then goes through to characterise pairing (39) as the unique product extending $\mu_{p^{\nu}} \otimes \mu_{p^{\nu}} \cong \mu_{p^{\nu}}^{\otimes 2}$. This gives (b).

Finally, the proof of [31, Corollary 7.2.4] for $f: \mathcal{X} \rightarrow S$ - and hence $c=-1, n=1, m=0$ - goes through to give (c).

Corollary 5. There is a commutative diagram of duality isomorphisms

$$
\begin{array}{cc}
R \Gamma\left(S, \mathbb{Z}(1) / p^{\nu}\right) & R \Gamma\left(\mathcal{X}, \mathbb{Z}(1) / p^{\nu}\right) \\
\mathbf{A V}(S, 1) \downarrow & \mathbf{A V}(\mathcal{X}, 1) \downarrow \\
\hat{R} \Gamma_{c}\left(S, \mathbb{Z} / p^{\nu}\right)^{*}[-3] & \longrightarrow \hat{R} \Gamma_{c}\left(\mathcal{X}, \mathbb{Z}(1) / p^{\nu}\right)^{*}[-5]
\end{array}
$$

where the top (resp., bottom) horizontal map is induced by $f^{*}$ (resp., $\operatorname{Tr}_{f}^{0}$ ) in diagram (40).

\section{Isolating the $H^{1}$-part}

Throughout this section, $\mathcal{X}$ denotes an arithmetic surface, and we use the notation defined in the introduction. The formulation of the special-value conjecture [6, Conjecture 5.12] for $\zeta(\mathcal{X}, s)$ at $s=1$ involves the fundamental line

$$
\Delta(\mathcal{X} / \mathbb{Z}, 1):=\operatorname{det}_{\mathbb{Z}} R \Gamma_{W, c}(\mathcal{X}, \mathbb{Z}(1)) \otimes_{\mathbb{Z}} \operatorname{det}_{\mathbb{Z}} R \Gamma\left(\mathcal{X}_{Z a r}, L \Omega_{\mathcal{X} / \mathbb{Z}}^{<1}\right)
$$

and the exact triangle $[6,(5)]$

$$
R \Gamma\left(\mathcal{X}_{Z a r}, L \Omega_{\mathcal{X} / \mathbb{Z}}^{<1}\right)_{\mathbb{R}}[-2] \rightarrow R \Gamma_{\text {ar }, c}(\mathcal{X}, \tilde{\mathbb{R}}(1)) \rightarrow R \Gamma_{W, c}(\mathcal{X}, \mathbb{Z}(1))_{\mathbb{R}} \rightarrow,
$$

which induces the trivialisation $\lambda_{\infty}$ of the determinant of $\Delta(\mathcal{X} / \mathbb{Z}, 1)_{\mathbb{R}}$. For each complex in triangle (42), we shall define in this section a corresponding complex for the relative $H^{1}$-motive of the morphism $f: \mathcal{X} \rightarrow S$ and obtain a corresponding description of $\zeta\left(H^{1}, s\right)$ at $s=1$. In the absence of a suitable triangulated category of motivic complexes $D M$ with a motivic $t$-structure, we isolate the relative $H^{1}$-motive in an ad hoc way as an object of the derived category of étale sheaves on $S$. More precisely, by [14, Corollary 3.2] we have $R^{i} f_{*} \mathbb{G}_{m}=0$ for $i \geq 2$ and

$$
P=\operatorname{Pic}_{\mathcal{X} / S}:=R^{1} f_{*} \mathbb{G}_{m}
$$

is the relative Picard functor studied in, for example, [29]. There is a truncation triangle

$$
\mathbb{G}_{m} \rightarrow R f_{*} \mathbb{G}_{m} \rightarrow P[-1] \rightarrow
$$


and we define a complex of étale sheaves $P^{0}$ by the exact triangle

$$
P^{0} \rightarrow P \stackrel{\operatorname{deg}}{\longrightarrow} \mathbb{Z} \rightarrow
$$

where the degree map is discussed in, for example, [14, Section 4]. The complex $P^{0}$ serves as a substitute for the relative $H^{1}$-motive, and we will define Weil-étale and Weil-Arakelov complexes associated to it according to the following table. The first column refers to definitions made in [6]. In particular, $\overline{\mathcal{X}}$ and $\bar{S}$ denote the Artin-Verdier compactifications of $\mathcal{X}$ and $S$, respectively. If $f$ has a section, the complexes in the right-hand column are direct summands of those in the left-hand column. In general, the exact triangles (43) and (44) will induce corresponding exact triangles for the Weil-étale complexes associated to $\mathbb{Z}(1), \mathbb{Z}, P$ and $P^{0}$ on $S$.

\begin{tabular}{c|c}
$R \Gamma_{W}(\overline{\mathcal{X}}, \mathbb{Z}(1))$ & $R \Gamma_{W}\left(\bar{S}, P^{0}\right)[-2]$ \\
\hline$R \Gamma_{W}\left(\mathcal{X}_{\infty}, \mathbb{Z}(1)\right)$ & $R \Gamma_{W}\left(S_{\infty}, P^{0}\right)[-2]$ \\
\hline$R \Gamma_{W, c}(\mathcal{X}, \mathbb{Z}(1))$ & $R \Gamma_{W, c}\left(S, P^{0}\right)[-2]$ \\
\hline$R \Gamma_{\text {ar }, c}(\mathcal{X}, \tilde{\mathbb{R}}(1))$ & $R \Gamma_{\mathrm{ar}, c}\left(S, P_{\mathbb{R}}^{0}\right)[-2]$ \\
\hline$R \Gamma\left(\mathcal{X}_{Z a r}, L \Omega_{\mathcal{X} / \mathbb{Z}}^{<1}\right)$ & $H^{1}\left(\mathcal{X}, \mathcal{O}_{\mathcal{X}}\right)[-1]$
\end{tabular}

The precise definition of the terms in the right-hand column (from top to bottom) will be given in the proofs of Lemmas 8 and 9 and in formulas (61) and (64), respectively.

Lemma 8. If $\operatorname{Br}(\mathcal{X})$ is finite, then $R \Gamma_{W}\left(\bar{S}, P^{0}\right)$ is a perfect complex of abelian groups satisfying a duality

$$
R \Gamma_{W}\left(\bar{S}, P^{0}\right) \stackrel{\sim}{\longrightarrow} R \operatorname{Hom}_{\mathbb{Z}}\left(R \Gamma_{W}\left(\bar{S}, P^{0}\right), \mathbb{Z}[-1]\right)
$$

Its cohomology is given by

$$
H_{W}^{i}\left(\bar{S}, P^{0}\right)= \begin{cases}\operatorname{Pic}^{0}(\mathcal{X}) / \operatorname{Pic}\left(\mathcal{O}_{F}\right), & i=0 \\ H^{1}\left(\bar{S}, P^{0}\right) \oplus \operatorname{Hom}_{\mathbb{Z}}\left(\operatorname{Pic}^{0}(\mathcal{X}), \mathbb{Z}\right), & i=1, \\ \left(\operatorname{Pic}^{0}(\mathcal{X})_{t o r} / \operatorname{Pic}\left(\mathcal{O}_{F}\right)\right)^{*}, & i=2,\end{cases}
$$

where $H^{1}\left(\bar{S}, P^{0}\right)=H^{1}\left(\bar{S}_{\text {et }}, P^{0}\right)$ is a finite abelian group of cardinality $\# \operatorname{Br}(\overline{\mathcal{X}}) \delta^{2}$ and $\operatorname{Br}(\overline{\mathcal{X}})$ is defined by the exact sequence

$$
0 \rightarrow \operatorname{Br}(\overline{\mathcal{X}}) \rightarrow \operatorname{Br}(\mathcal{X}) \rightarrow \bigoplus_{v \text { real }} \operatorname{Br}\left(\mathcal{X}_{F_{v}}\right)
$$


In particular, $\operatorname{Br}(\overline{\mathcal{X}})$ coincides with $\operatorname{Br}(\mathcal{X})$ if $F$ has no real places and is a subgroup of $\operatorname{Br}(\mathcal{X})$ of co-exponent 2 in general.

Proof. According to their definition in [6, Appendix A], the Artin-Verdier étale topoi of $\mathcal{X}$ and $S$ fit into a commutative diagram of morphisms of topoi

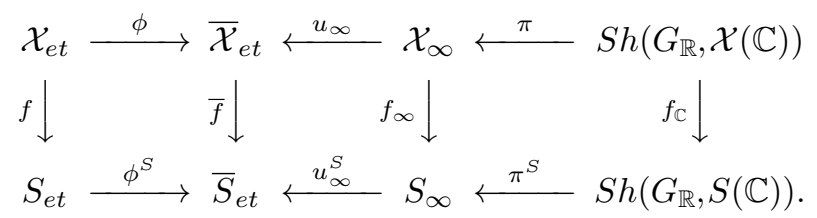

Applying $R \bar{f}_{*}$ to the defining exact triangle [6, Appendix A, Corollary 6.8] of the complex $\mathbb{Z}(1)^{\overline{\mathcal{X}}}$, we obtain a commutative diagram of exact triangles

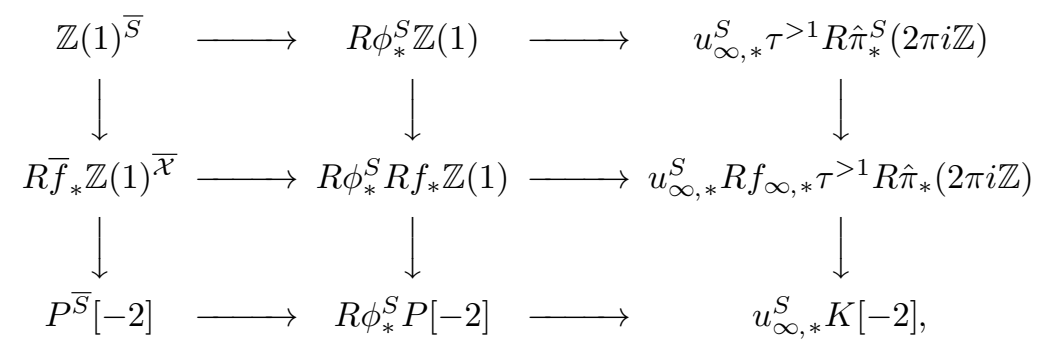

where the middle vertical triangle is induced by triangle (43) and the left (resp., right) vertical triangle defines $P^{\bar{S}}$ (resp., $\left.K\right)$. Since $R \hat{\pi}_{*}(2 \pi i \mathbb{Z})$ (resp., $R \hat{\pi}_{*}^{S}(2 \pi i \mathbb{Z})$ ) is a complex of sheaves supported on $\mathcal{X}(\mathbb{R})$ (resp., $S(\mathbb{R})$ ) with stalk $H^{j}\left(G_{\mathbb{R}}, 2 \pi i \mathbb{Z}\right)=\mathbb{Z} / 2 \mathbb{Z}$ in odd degrees $j$, and vanishing in even degrees, it follows that the top two complexes in the right-hand column are supported in degrees $\geq 3$. This gives exactness of the columns in the diagram

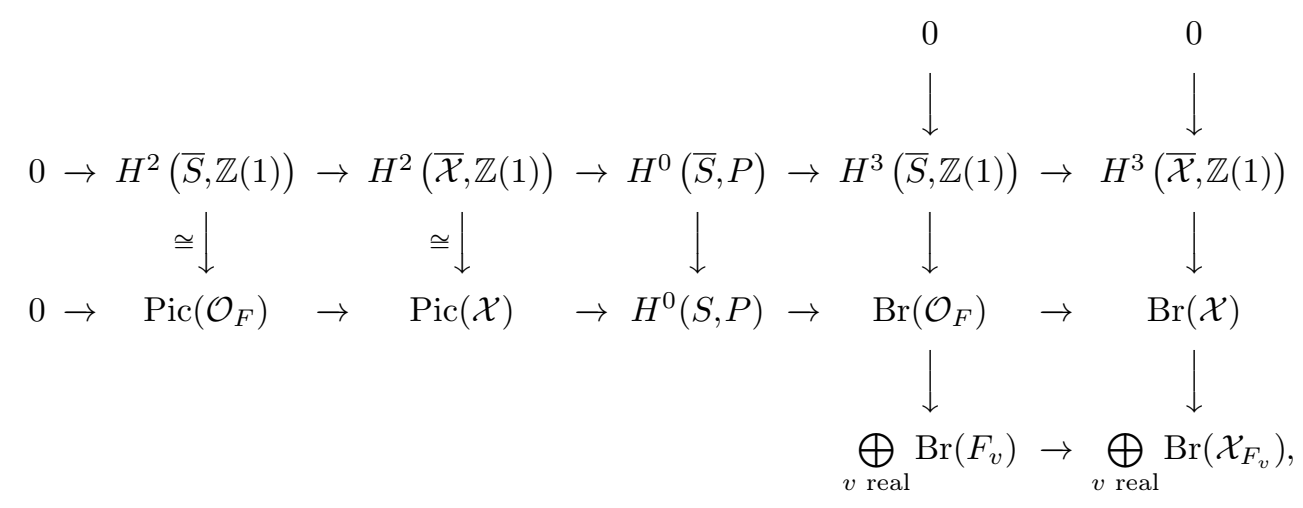


which is the map of long exact cohomology sequences induced by the left two columns in diagram (47). Here we use the isomorphism (see the proof of Lemma 11)

$$
H^{3}\left(\mathcal{X}_{\infty}, \tau^{>1} R \hat{\pi}_{*}(2 \pi i \mathbb{Z})\right) \cong H^{3}\left(G_{\mathbb{R}}, \mathcal{X}(\mathbb{C}),(2 \pi i) \mathbb{Z}\right) \cong H^{3}\left(\mathcal{X}_{\mathbb{R}, e t}, \mathbb{Z}(1)\right) \cong \bigoplus_{v \text { real }} \operatorname{Br}\left(\mathcal{X}_{F_{v}}\right)
$$

and similarly for $S_{\infty}$. We deduce that $\operatorname{Br}(\bar{S}):=H^{3}(\bar{S}, \mathbb{Z}(1))=0$, and hence

$$
H^{0}(\bar{S}, P) \cong \operatorname{Pic}(\mathcal{X}) / \operatorname{Pic}\left(\mathcal{O}_{F}\right)
$$

and that $H^{3}(\overline{\mathcal{X}}, \mathbb{Z}(1))$ coincides with the group $\operatorname{Br}(\overline{\mathcal{X}})$ defined in expression (45). The continuation of the top long exact sequence gives

$$
\begin{array}{r}
0 \rightarrow H^{3}(\overline{\mathcal{X}}, \mathbb{Z}(1)) \rightarrow H^{1}(\bar{S}, P) \rightarrow H^{4}(\bar{S}, \mathbb{Z}(1)) \quad \rightarrow \quad H^{4}(\overline{\mathcal{X}}, \mathbb{Z}(1)) \\
\cong \downarrow \quad \cong \downarrow \\
H^{0}(\bar{S}, \mathbb{Z})^{*} \stackrel{\operatorname{deg}^{*}}{\longrightarrow} H^{2}(\overline{\mathcal{X}}, \mathbb{Z}(1))^{*},
\end{array}
$$

where the vertical duality isomorphisms follow from [6, Proposition 3.4] for both $S$ and $\mathcal{X}$, taking into account the compatibility of dualities in Corollary 5. Since we have a commutative diagram

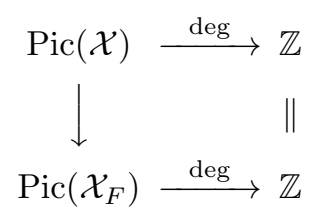

with surjective vertical map, the cokernel of both degree maps is $\mathbb{Z} / \delta \mathbb{Z}$, where $\delta$ is the greatest common divisor of the degrees of all divisors on $\mathcal{X}_{F}$ - that is, the index of $\mathcal{X}_{F}$. Hence there is an exact sequence

$$
0 \rightarrow \operatorname{Br}(\overline{\mathcal{X}}) \rightarrow H^{1}(\bar{S}, P) \rightarrow \mathbb{Z} / \delta \mathbb{Z} \rightarrow 0
$$

We can similarly extend triangle (44) to the Artin-Verdier compactification. The composite map

$$
(2 \pi i \mathbb{Z}) \rightarrow R f_{\mathbb{C}, *}(2 \pi i \mathbb{Z}) \rightarrow R^{2} f_{\mathbb{C}, *}(2 \pi i \mathbb{Z})[-2] \stackrel{\operatorname{deg}}{\longrightarrow} \mathbb{Z}[-2]
$$

in $S h\left(G_{\mathbb{R}}, S(\mathbb{C})\right)$ clearly vanishes, and the commutativity of the right-hand square in diagram (46) then shows that the degree map on the middle row of diagram (47) factors through the lower row - that is, we obtain a commutative diagram with exact rows and columns 


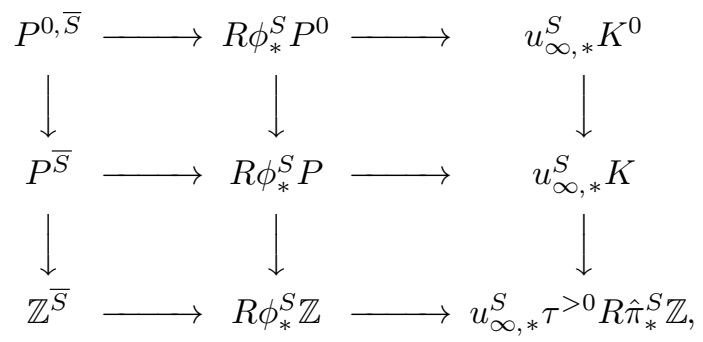

where the lower row is the defining exact triangle [6, Appendix A, Corollary 6.8] of the complex $\mathbb{Z}^{\bar{S}}$ and the left-hand column defines $P^{0, \bar{S}}$. Note that in fact $\mathbb{Z}^{\bar{S}}=\mathbb{Z}$. The long exact cohomology sequence of the left-hand column is

$$
0 \rightarrow H^{0}\left(\bar{S}, P^{0}\right) \rightarrow H^{0}(\bar{S}, P) \stackrel{\operatorname{deg}}{\longrightarrow} \mathbb{Z} \rightarrow H^{1}\left(\bar{S}, P^{0}\right) \rightarrow H^{1}(\bar{S}, P) \rightarrow 0
$$

since $H^{1}(\bar{S}, \mathbb{Z})=H^{1}(S, \mathbb{Z})=0$. From formula (48) we obtain

$$
H^{0}\left(\bar{S}, P^{0}\right) \cong \operatorname{Pic}^{0}(\mathcal{X}) / \operatorname{Pic}\left(\mathcal{O}_{F}\right)
$$

and from formula $(49)$ that $H^{1}\left(\bar{S}, P^{0}\right)$ has cardinality $\# \operatorname{Br}(\overline{\mathcal{X}}) \delta^{2}$.

In order to compute $H^{i}\left(\bar{S}, P^{0}\right)$ in degrees $\geq 2$, we prove a torsion duality for $P^{0} / p^{\nu}$ for any prime $p$ : the isomorphism $\mathbf{A V}\left(\bar{S}, P^{0}\right)$ in diagram (55). Following the proof of $[6$, Proposition 3.4], this then implies

$$
H^{i}\left(\bar{S}, P^{0}\right) \cong H^{2-i}\left(\bar{S}, P^{0}\right)^{*}
$$

for $i \geq 2$, in particular $H^{i}\left(\bar{S}, P^{0}\right)=0$ for $i \geq 3$. Following [6, Definition 3.6], we then define $R \Gamma_{W}\left(\bar{S}, P^{0}\right)$ by an exact triangle

$$
R \operatorname{Hom}\left(R \Gamma_{W}\left(\bar{S}, P^{0}\right), \mathbb{Q}[-2]\right) \rightarrow R \Gamma\left(\bar{S}, P^{0}\right) \rightarrow R \Gamma_{W}\left(\bar{S}, P^{0}\right) \rightarrow
$$

and obtain the cohomology groups described in Lemma 8. Conjecture $\mathbf{L}\left(\mathcal{X}_{e t}, 1\right)$ entering into the definition of Weil-étale modifications in [6, Section 3] reduces to the finiteness of $\operatorname{Br}(\mathcal{X})=H^{3}\left(\mathcal{X}_{\text {et }}, \mathbb{Z}(1)\right)$ and finite generation of $\operatorname{Pic}(\mathcal{X})$, which is well known.

Fix a prime number $p$ and consider the following commutative diagram with exact columns: 


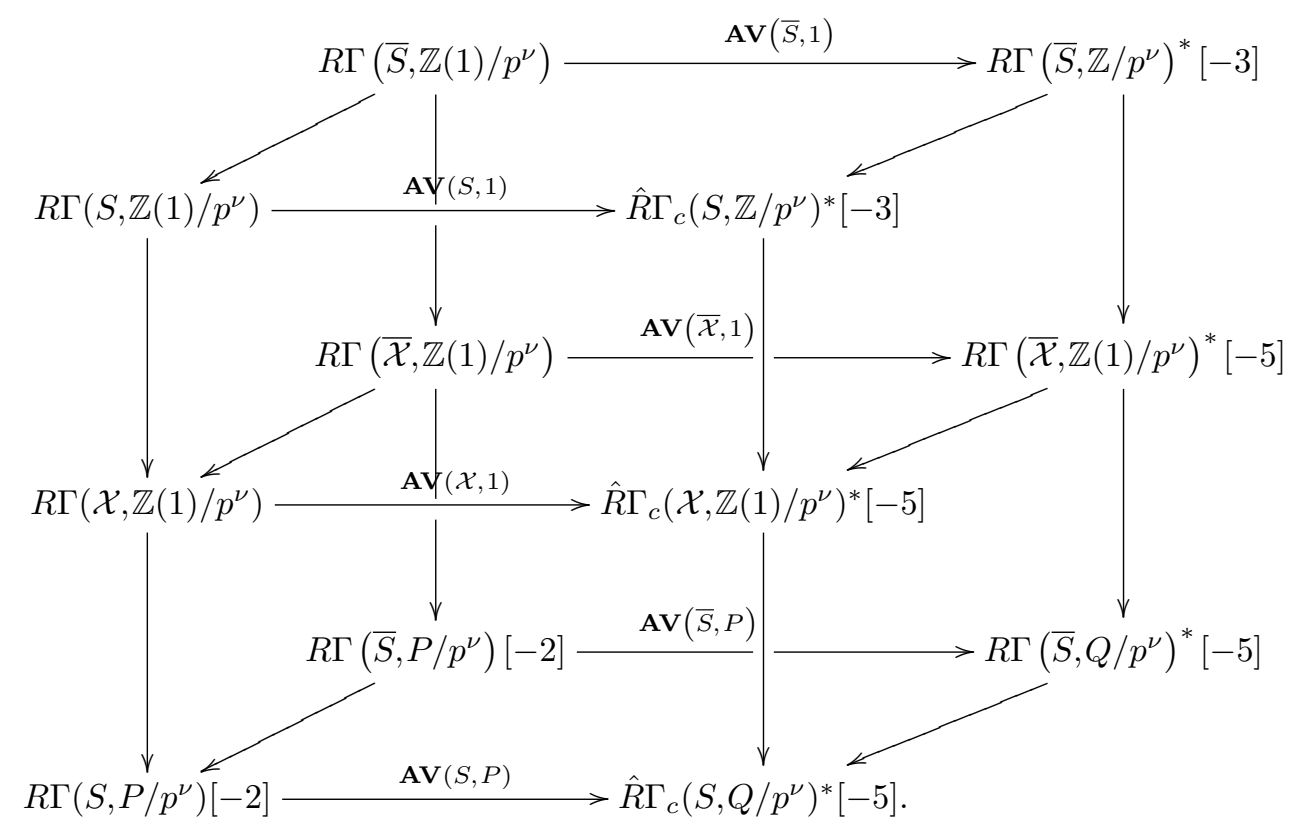

The top-left commutative square is induced by the top-left square in diagram (47) after applying $-\otimes^{L} \mathbb{Z} / p^{\nu} \mathbb{Z}$. The top and middle commutative squares are those in the proof of [6, Theorem 6.24] for $S$ and $\mathcal{X}$, respectively. The front commutative diagram of exact triangles arises from Corollary 5, where we define the complex $Q^{\bar{S}}$ on $\bar{S}$ by the exact triangle

$$
Q^{\bar{S}} \rightarrow R \bar{f}_{*} \mathbb{Z}(1)^{\overline{\mathcal{X}} \stackrel{\operatorname{deg}}{\longrightarrow}} \mathbb{Z}^{\bar{S}}[-2] \rightarrow
$$

and denote by $Q$ its restriction to $S$. Note that there is also an exact triangle

$$
\mathbb{Z}(1)^{\bar{S}} \rightarrow Q^{\bar{S}} \rightarrow P^{0, \bar{S}}[-2] \rightarrow
$$

It follows then from Corollary 5 and [6, Theorem 6.24] that all arrows labeled $\mathbf{A V}$ in diagram (52) are quasi-isomorphisms. We finally obtain an isomorphism of exact triangles

$$
\begin{aligned}
& R \Gamma\left(\bar{S}, \mathbb{Z}(1) / p^{\nu}\right) \quad \longrightarrow \quad R \Gamma\left(\bar{S}, Q / p^{\nu}\right) \quad \longrightarrow R \Gamma\left(\bar{S}, P^{0} / p^{\nu}\right)[-2] \\
& \operatorname{AV}(\bar{S}, 1) \downarrow \quad \operatorname{AV}(\bar{S}, P)^{*}[-5] \downarrow \quad \mathbf{A V}\left(\bar{S}, P^{0}\right)[-2] \downarrow \\
& R \Gamma\left(\bar{S}, \mathbb{Z} / p^{\nu}\right)^{*}[-3] \longrightarrow R \Gamma\left(\bar{S}, P / p^{\nu}\right)^{*}[-3] \longrightarrow R \Gamma\left(\bar{S}, P^{0} / p^{\nu}\right)^{*}[-3],
\end{aligned}
$$

where the top (resp., bottom) row is induced by triangle (54) (resp., the left-hand column in diagram (50)) and the commutativity of the left square again follows from Corollary 5. This concludes the proof. 
The $H^{1}$-part $R \Gamma_{W}\left(S_{\infty}, P^{0}\right)$ of the complex $R \Gamma_{W}\left(\mathcal{X}_{\infty}, \mathbb{Z}(1)\right)$ defined in [6, Definition 3.23] is specified by two exact triangles

$$
R \Gamma_{W}\left(S_{\infty}, \mathbb{Z}(1)\right) \rightarrow R \Gamma_{W}\left(\mathcal{X}_{\infty}, \mathbb{Z}(1)\right) \rightarrow R \Gamma_{W}\left(S_{\infty}, P\right)[-2]
$$

and

$$
R \Gamma_{W}\left(S_{\infty}, P^{0}\right) \rightarrow R \Gamma_{W}\left(S_{\infty}, P\right) \rightarrow R \Gamma_{W}\left(S_{\infty}, \mathbb{Z}\right)
$$

and its cohomology is computed by the following lemma:

Lemma 9. We have

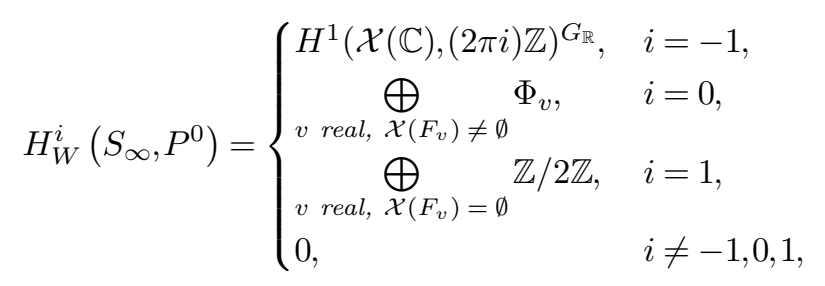

where $\Phi_{v}=J_{F}\left(F_{v}\right) / J_{F}\left(F_{v}\right)^{0}$ is the component group as defined in the introduction. Moreover,

$$
\frac{\# H_{W}^{0}\left(S_{\infty}, P^{0}\right)}{\# H_{W}^{1}\left(S_{\infty}, P^{0}\right)}=\prod_{v \text { real }} \frac{\# \Phi_{v}}{\delta_{v}^{\prime} \delta_{v}}
$$

Proof. The topological space $\mathcal{X}_{\infty}=\mathcal{X}(\mathbb{C}) / G_{\mathbb{R}}$ is a 2-manifold (posssibly nonorientable, possibly with boundary $\mathcal{X}(\mathbb{R})$ ) and hence has sheaf-cohomological dimension $\leq 2$. The complex

$$
i_{\infty}^{*} \mathbb{Z}(1):=\tau^{\leq 1} R \pi_{*}(2 \pi i) \mathbb{Z}
$$

of [6, Definition 3.23] is concentrated in degrees 0 and 1 and $R^{1} \pi_{*}(2 \pi i) \mathbb{Z}$ is supported on the closed subset $\mathcal{X}(\mathbb{R})$, a union of circles, hence of cohomological dimension 1 . It follows that $R \Gamma_{W}\left(\mathcal{X}_{\infty}, \mathbb{Z}(1)\right)$ is concentrated in degrees $\leq 2$.

Triangles (56) and (57) are direct sums over the infinite places $v \in S_{\infty}$, and we denote the respective direct summands by an index $v$. If $v$ is a complex place or a real place with $\mathcal{X}\left(F_{v}\right) \neq \emptyset$, then $f_{\infty, v}$ has a section and the exact triangle $[6,(47)]$

$$
R \Gamma_{W}\left(\mathcal{X}_{\infty}, \mathbb{Z}(1)\right)_{v} \rightarrow R \Gamma\left(G_{\mathbb{R}}, \mathcal{X}(\mathbb{C}),(2 \pi i) \mathbb{Z}\right)_{v} \rightarrow R \Gamma\left(\mathcal{X}(\mathbb{R}), \tau^{>1} R \hat{\pi}_{*}(2 \pi i) \mathbb{Z}\right)_{v}
$$

splits into a direct sum of its $H^{i}$-parts for $i=0,1,2$. The last term being concentrated in degrees $\geq 3$, we find

$$
R \Gamma_{W}\left(S_{\infty}, P^{0}\right)_{v}[-2] \cong \tau^{\leq 2} R \Gamma\left(G_{\mathbb{R}}, H^{1}\left(\mathcal{X}\left(F_{v} \otimes_{\mathbb{R}} \mathbb{C}\right),(2 \pi i) \mathbb{Z}\right)[-1]\right)
$$

for the $H^{1}$-part. The group $H^{1}\left(G_{\mathbb{R}}, H^{1}\left(\mathcal{X}\left(F_{v} \otimes_{\mathbb{R}} \mathbb{C}\right),(2 \pi i) \mathbb{Z}\right)\right)$ is isomorphic to $\Phi_{v}$, as can be verified easily by taking the $G_{\mathbb{R}}$-cohomology of the exponential sequence for the abelian variety $J_{F}\left(F_{v} \otimes_{\mathbb{R}} \mathbb{C}\right)$. 
If $v$ is a real place with $\mathcal{X}\left(F_{v}\right)=\emptyset$, an analysis of the spectral sequence

$$
H^{p}\left(G_{\mathbb{R}}, H^{q}\left(\mathcal{X}\left(F_{v} \otimes_{\mathbb{R}} \mathbb{C}\right),(2 \pi i) \mathbb{Z}\right)\right) \Rightarrow H^{p+q}\left(G_{\mathbb{R}}, \mathcal{X}\left(F_{v} \otimes_{\mathbb{R}} \mathbb{C}\right),(2 \pi i) \mathbb{Z}\right)
$$

reveals that

$$
H_{W}^{i}\left(S_{\infty}, P\right)_{v}= \begin{cases}H^{1}\left(\mathcal{X}\left(F_{v} \otimes_{\mathbb{R}} \mathbb{C}\right),(2 \pi i) \mathbb{Z}\right)^{G_{\mathbb{R}}}, & i=-1, \\ \operatorname{ker}\left(H^{2}\left(\mathcal{X}\left(F_{v} \otimes_{\mathbb{R}} \mathbb{C}\right),(2 \pi i) \mathbb{Z}\right) \cong \mathbb{Z} \rightarrow \mathbb{Z} / 2 \mathbb{Z}\right), & i=0 .\end{cases}
$$

Here we use the fact that the end term is concentrated in degrees $\leq 2$ and that for $i \geq 1$,

$$
\# \Phi_{v}=\# H^{i}\left(G_{\mathbb{R}}, H^{1}\left(\mathcal{X}\left(F_{v} \otimes_{\mathbb{R}} \mathbb{C}\right),(2 \pi i) \mathbb{Z}\right)\right) \in\{1,2\}
$$

by [12, Propositions 3.3 and 1.3]. The long exact sequence associated to triangle (57) then gives the computation (58).

To show equation (59), first note that $\mathcal{X}\left(F_{v}\right)=\emptyset$ if and only if $\delta_{v}=2$; otherwise, $\delta_{v}=1$. Since $\delta_{v}^{\prime} \mid \delta_{v}$, this shows equation (59) at places where $\mathcal{X}\left(F_{v}\right) \neq \emptyset$. If $\mathcal{X}\left(F_{v}\right)=\emptyset$, we have $\# \Phi_{v}=1$ or 2 , according to whether the genus $g$ of $\mathcal{X}$ is even or odd, by [12, Proposition 3.3], and we also have $\delta_{v}^{\prime}=1$ or 2 , according to whether $g$ is even or odd, by [22]. More precisely, $g$ even implies $\delta_{v}^{\prime}=1$ by [22, Theorem 7a], and $\delta_{v}^{\prime}=1$ implies $\delta_{v}=2 \delta_{v}^{\prime}$, which implies $(g-1) / \delta_{v}^{\prime}=g-1$ odd - that is, $g$ even - by [22, Theorem $\left.7 \mathrm{c}\right]$. These results hold for arbitrary local fields by [28, p. 1126]. This shows equation (59) at places where $\mathcal{X}\left(F_{v}\right)=\emptyset$.

The complex $R \Gamma_{W, c}\left(S, P^{0}\right)$ is defined by an exact triangle

$$
R \Gamma_{W, c}\left(S, P^{0}\right) \rightarrow R \Gamma_{W}\left(\bar{S}, P^{0}\right) \rightarrow R \Gamma_{W}\left(S_{\infty}, P^{0}\right) \rightarrow
$$

and Lemmas 8 and 9 imply an exact sequence

$$
\begin{aligned}
0 & \rightarrow H^{1}(\mathcal{X}(\mathbb{C}),(2 \pi i) \mathbb{Z})^{G_{\mathbb{R}}} \rightarrow H_{W, c}^{0}\left(S, P^{0}\right) \rightarrow \operatorname{Pic}^{0}(\mathcal{X}) / \operatorname{Pic}\left(\mathcal{O}_{F}\right) \rightarrow \\
& \rightarrow H_{W}^{0}\left(S_{\infty}, P^{0}\right) \rightarrow H_{W, c}^{1}\left(S, P^{0}\right) \rightarrow H^{1}\left(\bar{S}, P^{0}\right) \oplus \operatorname{Hom}_{\mathbb{Z}}\left(\operatorname{Pic}^{0}(\mathcal{X}), \mathbb{Z}\right) \rightarrow \\
& \rightarrow H_{W}^{1}\left(S_{\infty}, P^{0}\right) \rightarrow H_{W, c}^{2}\left(S, P^{0}\right) \rightarrow\left(\operatorname{Pic}^{0}(\mathcal{X})_{\text {tor }} / \operatorname{Pic}\left(\mathcal{O}_{F}\right)\right)^{*} \rightarrow 0 .
\end{aligned}
$$

Define $\mathbb{Z}$-bases $b_{B}, b_{d R}$ and $b_{M}$ by

$$
\begin{aligned}
\mathbb{Z} \cdot b_{B} & =\operatorname{det}_{\mathbb{Z}} H^{1}(\mathcal{X}(\mathbb{C}),(2 \pi i) \mathbb{Z})^{G_{\mathbb{R}}} \\
\mathbb{Z} \cdot b_{d R} & =\operatorname{det}_{\mathbb{Z}} H^{1}\left(\mathcal{X}, \mathcal{O}_{\mathcal{X}}\right) \\
\mathbb{Z} \cdot b_{M} & =\operatorname{det}_{\mathbb{Z}} \operatorname{Pic}^{0}(\mathcal{X}) / \operatorname{Pic}^{0}(\mathcal{X})_{\text {tor }}
\end{aligned}
$$

Note here that $H^{1}\left(\mathcal{X}, \mathcal{O}_{\mathcal{X}}\right)$ might have nontrivial torsion (if $f$ is not cohomologically flat), and its determinant has to be understood as that of a perfect complex. Then the fundamental line of the $H^{1}$-part

$$
\Delta\left(S / \mathbb{Z}, P^{0}\right):=\operatorname{det}_{\mathbb{Z}} R \Gamma_{W, c}\left(S, P^{0}\right) \otimes_{\mathbb{Z}} \operatorname{det}_{\mathbb{Z}}^{-1} H^{1}\left(\mathcal{X}, \mathcal{O}_{\mathcal{X}}\right),
$$


when viewed inside $\Delta\left(S / \mathbb{Z}, P^{0}\right)_{\mathbb{Q}}$, has $\mathbb{Z}$-basis

$$
\Delta\left(S / \mathbb{Z}, P^{0}\right)=\mathbb{Z} \cdot b_{B} \cdot \frac{b_{M}}{T} \cdot \# H_{W}^{0}\left(S_{\infty}\right) \cdot \# H^{1}(\bar{S}) \cdot b_{M} \cdot \# H_{W}^{1}\left(S_{\infty}\right)^{-1} \cdot T^{-1} \cdot b_{d R}^{-1},
$$

where $T:=\# \operatorname{Pic}^{0}(\mathcal{X})_{t o r} / \operatorname{Pic}\left(\mathcal{O}_{F}\right)$ and we have left out coefficients $P^{0}$ for clarity.

We define

$$
R \Gamma_{\text {ar }, c}\left(S, P_{\mathbb{R}}^{0}\right):=\operatorname{Pic}^{0}(\mathcal{X})_{\mathbb{R}} \oplus \operatorname{Pic}^{0}(\mathcal{X})_{\mathbb{R}}[-1] .
$$

The $H^{1}$-part of the exact triangle (42) is an exact triangle

$$
H^{1}\left(\mathcal{X}, \mathcal{O}_{\mathcal{X}}\right)_{\mathbb{R}}[-3] \rightarrow \operatorname{Pic}^{0}(\mathcal{X})_{\mathbb{R}}[-2] \oplus \operatorname{Pic}^{0}(\mathcal{X})_{\mathbb{R}}[-3] \rightarrow R \Gamma_{W, c}\left(S, P^{0}\right)_{\mathbb{R}}[-2] \rightarrow
$$

whose associated long exact sequence

$$
0 \rightarrow \operatorname{Pic}^{0}(\mathcal{X})_{\mathbb{R}} \rightarrow H_{W, c}^{0}\left(S, P^{0}\right)_{\mathbb{R}} \rightarrow H^{1}\left(\mathcal{X}, \mathcal{O}_{\mathcal{X}}\right)_{\mathbb{R}} \rightarrow \operatorname{Pic}^{0}(\mathcal{X})_{\mathbb{R}} \rightarrow H_{W, c}^{1}\left(S, P^{0}\right)_{\mathbb{R}} \rightarrow 0
$$

involves the Deligne period isomorphism (see the discussion before Lemma 18)

$$
H^{1}(\mathcal{X}(\mathbb{C}),(2 \pi i) \mathbb{Z})_{\mathbb{R}}^{G_{\mathbb{R}}} \cong H^{1}\left(\mathcal{X}, \mathcal{O}_{\mathcal{X}}\right)_{\mathbb{R}}
$$

on $\mathcal{X}(\mathbb{C})$, the Arakelov intersection pairing

$$
\operatorname{Pic}^{0}(\mathcal{X})_{\mathbb{R}} \cong \operatorname{Hom}_{\mathbb{Z}}\left(\operatorname{Pic}^{0}(\mathcal{X}), \mathbb{R}\right)
$$

and the description of $H_{W, c}^{i}\left(S, P^{0}\right)$ given by sequence $(62)$. Define $\Omega(\mathcal{X}), R(\mathcal{X}) \in \mathbb{R}^{\times}$by

$$
b_{B}=\Omega(\mathcal{X}) \cdot b_{d R}
$$

under the determinant of isomorphism (66) and

$$
b_{M}=R(\mathcal{X}) \cdot b_{M}^{-1}
$$

under the determinant of pairing (67). Then the isomorphism

$$
\lambda_{\infty}: \mathbb{R} \cong \Delta\left(S / \mathbb{Z}, P^{0}\right)_{\mathbb{R}}
$$

induced by triangle (65) sends

$$
\frac{\# \operatorname{Br}(\overline{\mathcal{X}}) \cdot \delta^{2} \cdot \Omega(\mathcal{X}) \cdot R(\mathcal{X})}{\left(\#\left(\operatorname{Pic}^{0}(\mathcal{X})_{t o r} / \operatorname{Pic}\left(\mathcal{O}_{F}\right)\right)\right)^{2}} \cdot \prod_{v \text { real }} \frac{\# \Phi_{v}}{\delta_{v}^{\prime} \delta_{v}}
$$

to $\Delta\left(S / \mathbb{Z}, P^{0}\right)$, where we have used equations (63) and (59) and Lemma 8. Since $\zeta^{*}\left(H^{1}, 1\right)$ appears in the denominator of $\zeta^{*}(\mathcal{X}, 1)$, and since $C(\mathcal{X}, 1)=1$, our special-value conjecture for the $H^{1}$-part

$$
\lambda_{\infty}\left(\zeta^{*}\left(H^{1}, 1\right) \cdot \mathbb{Z}\right)=\Delta\left(S / \mathbb{Z}, P^{0}\right)
$$

is given by formula (6).

Although it seems eminently plausible, we were not able to show that the pairing induced on

$$
\operatorname{Pic}^{0}(\mathcal{X})_{\mathbb{R}}=\operatorname{im}\left(H_{c}^{2}(\mathcal{X}, \mathbb{R}(1)) \rightarrow H^{2}(\mathcal{X}, \mathbb{R}(1))\right)
$$


by the pairing $[6$, Proposition 2.1]

$$
H^{2}(\mathcal{X}, \mathbb{R}(1)) \otimes H_{c}^{2}(\mathcal{X}, \mathbb{R}(1)) \rightarrow H_{c}^{4}(\mathcal{X}, \mathbb{R}(2)) \rightarrow \mathbb{R}
$$

coincides with the Arakelov intersection pairing, discussed for example in [19]. We shall, however, assume this from now on, or equivalently, we simply restate our conjecture using the Arakelov intersection pairing. Since the Arakelov intersection pairing is negative definite on $\operatorname{Pic}^{0}(\mathcal{X})_{\mathbb{R}}[19$, Theorem 3.4, Proposition 3.3], hence nondegenerate, map (67) is indeed an isomorphism.

Remark 1. We briefly indicate the $H^{1}$-part of some of the other complexes defined in [6]. [6, Definitions 4.3, 4.5 and 4.13] lead to the definitions

$$
\begin{gathered}
R \Gamma_{\text {ar }}\left(\bar{S}, P^{0}\right):=R \Gamma_{W}\left(\bar{S}, P^{0}\right) \\
R \Gamma_{\text {ar }}\left(\bar{S}, P^{0} \otimes \tilde{\mathbb{R}} / \mathbb{Z}\right):=R \Gamma_{W}\left(\bar{S}, P^{0}\right) \otimes_{\mathbb{Z}}^{L} \mathbb{R} / \mathbb{Z} \\
R \Gamma_{\text {ar }, c}\left(S, P^{0} \otimes \tilde{\mathbb{R}} / \mathbb{Z}\right):=R \Gamma_{W, c}\left(S, P^{0}\right) \otimes_{\mathbb{Z}}^{L} \mathbb{R} / \mathbb{Z},
\end{gathered}
$$

where all complexes have to be regarded in the bounded derived category of locally compact abelian groups [6, Remark 4.16]. The duality of Lemma 8 implies a Pontryagin duality

$$
R \Gamma_{\mathrm{ar}}\left(\bar{S}, P^{0}\right)^{D} \cong R \Gamma_{\mathrm{ar}}\left(\bar{S}, P^{0} \otimes \tilde{\mathbb{R}} / \mathbb{Z}\right)[1]
$$

which is the $H^{1}$-part of $\left[6\right.$, Theorem 4.9]. The complex $R \Gamma_{\text {ar, } c}\left(S, P^{0} \otimes \tilde{\mathbb{R}} / \mathbb{Z}\right)$ has compact cohomology groups concentrated in degrees 0 and 1 . If $\mathcal{X}\left(F_{v}\right) \neq \emptyset$ for all real places $v$, its cohomology is given by a short exact sequence

$$
\begin{aligned}
0 \rightarrow\left(\prod_{v \mid \infty} J_{F}\left(F_{v}\right)\right) /\left(\operatorname{Pic}^{0}(\mathcal{X}) / \operatorname{Pic}\left(\mathcal{O}_{F}\right)\right)_{t o r} \rightarrow & H_{\text {ar }, c}^{0}\left(S, P^{0} \otimes \tilde{\mathbb{R}} / \mathbb{Z}\right) \rightarrow \\
& \rightarrow \operatorname{Pic}^{0}(\mathcal{X}) \otimes \mathbb{Z} / \mathbb{Z} \oplus H^{1}\left(\bar{S}, P^{0}\right) \rightarrow 0
\end{aligned}
$$

and an isomorphism

$$
H_{\mathrm{ar}, c}^{1}\left(S, P^{0} \otimes \tilde{\mathbb{R}} / \mathbb{Z}\right) \cong\left(\operatorname{Pic}^{0}(\mathcal{X}) / \operatorname{Pic}\left(\mathcal{O}_{F}\right)\right)^{D}
$$

As explained in the introduction of [6], an alternative way to state equation (68) is

$$
\zeta^{*}\left(H^{1}, 1\right)=\frac{\operatorname{vol}\left(H_{\mathrm{ar}, c}^{0}\left(S, P^{0} \otimes \tilde{\mathbb{R}} / \mathbb{Z}\right)\right)}{\operatorname{vol}\left(H_{\mathrm{ar}, c}^{1}\left(S, P^{0} \otimes \tilde{\mathbb{R}} / \mathbb{Z}\right)\right)},
$$

where one has to choose volume forms $b_{0}, b_{1}$ in the tangent spaces of the two groups so that $b_{0} \cdot b_{1}^{-1}=\operatorname{det}_{\mathbb{Z}} H^{1}\left(\mathcal{X}, \mathcal{O}_{\mathcal{X}}\right)$ under the isomorphism induced by triangle (65). 


\section{Relating the Brauer and the Tate-Shafarevich groups}

A key ingredient in the comparison of our conjecture (6) with the Birch and SwinnertonDyer conjecture is a precise formula relating the cardinalities of $\operatorname{Br}(\mathcal{X})$ and $\amalg\left(J_{F}\right)$. The essential ideas for the comparison of these two groups can already be found in Grothendieck's article [14], and his results imply that $\operatorname{Br}(\mathcal{X}) \cong \amalg\left(J_{F}\right)$ if, for example, $f$ has a section and $F$ is totally imaginary. The general case is considerably more complicated, and was studied by a number of authors until it was recently settled by Geisser [10]. Unfortunately, Geisser's formula still has the condition that $F$ is totally imaginary, so we give here a generalisation of his result without this condition and with a different proof. What makes both proofs eventually possible are the duality results of Saito [30].

For any place $v$ of $F$ we denote by $\delta_{v}$ (resp., $\delta_{v}^{\prime}$ ) the index (resp., period) of $\mathcal{X}_{F_{v}}$ over $F_{v}$ - that is, the cardinalities of the cokernel of $\operatorname{Pic}\left(\mathcal{X}_{F_{v}}\right) \stackrel{\text { deg }}{\longrightarrow} \mathbb{Z}$ (resp., $\left.P\left(F_{v}\right) \stackrel{\text { deg }}{\longrightarrow} \mathbb{Z}\right)$. Then we have

$$
\delta_{v}^{\prime}\left|\delta_{v}\right| \delta
$$

and $\delta_{v} / \delta_{v}^{\prime} \in\{1,2\}$ for all places $v[28$, p. 1126]. We define

$$
\begin{aligned}
\alpha & :=\# \operatorname{coker}\left(\operatorname{Pic}^{0}\left(\mathcal{X}_{F}\right) \rightarrow J_{F}(F)\right) \\
& =\# \operatorname{coker}\left(H^{0}\left(\bar{S}, P^{0}\right) \rightarrow J_{F}(F)\right),
\end{aligned}
$$

where the equality holds because $H^{0}\left(\bar{S}, P^{0}\right)=\operatorname{Pic}^{0}(\mathcal{X}) / \operatorname{Pic}\left(\mathcal{O}_{F}\right) \rightarrow \operatorname{Pic}^{0}\left(\mathcal{X}_{F}\right)$ is surjective.

Proposition 5.1. Let $F$ be any number field, $f: \mathcal{X} \rightarrow \operatorname{Spec}\left(\mathcal{O}_{F}\right)$ an arithmetic surface and $J_{F}$ the Jacobian of $\mathcal{X}_{F}$. If $\operatorname{Br}(\mathcal{X})$ is finite, then

$$
\# \operatorname{Br}(\overline{\mathcal{X}}) \delta^{2}=\frac{\prod_{v} \delta_{v}^{\prime} \delta_{v}}{\alpha^{2}} \# \amalg\left(J_{F}\right)
$$

where the product is over all places $v$ of $F$ and $\operatorname{Br}(\overline{\mathcal{X}})$ was defined in Lemma 8.

Remark 2. As in [10, Corollary 1.2], it follows that the cardinality of $\operatorname{Br}(\overline{\mathcal{X}})$ is a square if it is finite.

Proof. For each finite place $v$ of $F$, we denote by $S_{v}=\bar{S}_{v}$ the spectrum of the Henselisation of $S$ at the closed point $v$ and by $F_{v}$ its field of fractions. If $v \in S_{\infty}$ is an infinite place of $F$, we denote by $F_{v} \subseteq \bar{F}$ the fixed field of a chosen decomposition group of $v$ in $\operatorname{Gal}(\bar{F} / F)$ and by $\bar{S}_{v}$ the local topos that is glued from $\operatorname{Spec}\left(F_{v}\right)_{e t}$ and $\operatorname{Shv}(v)=\operatorname{Set}$. Then there is a morphism of topoi $\bar{S}_{v} \rightarrow \bar{S}_{e t}$, and for a complex of sheaves $\mathcal{F}$ on $\bar{S}_{e t}$, the group $H^{i}\left(\bar{S}_{v}, \mathcal{F}\right)=H^{i}(v, \mathcal{F})=\mathcal{H}^{i}(\mathcal{F})_{v}$ is the stalk of $\mathcal{H}^{i}(\mathcal{F})$ at the point $v$ of the topos $\bar{S}_{e t}$. 
Let $\eta: \operatorname{Spec}(F) \rightarrow \bar{S}$ be the inclusion of the generic point, and define a commutative diagram of complexes on $\bar{S}$ with exact rows and columns

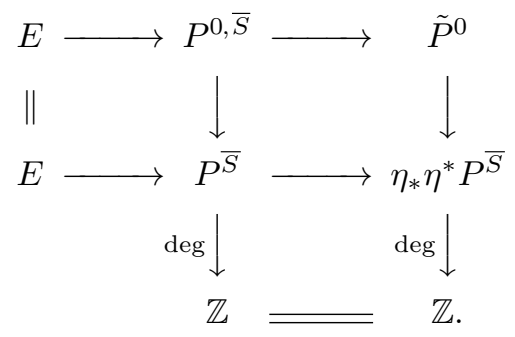

Note that $\eta^{*} P^{0, \bar{S}}$ is the sheaf represented by the Jacobian $J_{F}$, and $\mathcal{H}^{0}\left(\tilde{P}^{0}\right)=\eta_{*} J_{F}$ is the sheaf represented by the Néron model of $J_{F}$ over $S$.

Lemma 10. The complex $E$ is a sum of skyscraper sheaves in degrees 0 and 1 . We have

$$
\mathcal{H}^{0}(E) \cong \bigoplus_{v \in \Sigma_{f}} E_{v}
$$

where $\Sigma_{f}$ is the set of finite places of $F$ where $f$ is not smooth, and

$$
\mathcal{H}^{1}(E) \cong \bigoplus_{v \in S_{\infty}} \mathbb{Z} / \delta_{v} \mathbb{Z}
$$

Moreover, for $v \in \Sigma_{f}$ we have $H^{1}\left(S_{v}, E_{v}\right) \cong \mathbb{Z} / \delta_{v} \mathbb{Z}$ and hence

$$
H^{1}(\bar{S}, E) \cong \bigoplus_{v \in \Sigma} \mathbb{Z} / \delta_{v} \mathbb{Z}
$$

where $\Sigma=\Sigma_{f} \cup S_{\infty}$.

Proof. The restriction of the middle row of diagram (71) to $S$ is a short exact sequence of sheaves concentrated in degree 0 and has been analysed by Grothendieck [14, (4.10) bis]. The restriction of $E$ to $S$ is a sum over $v \in \Sigma_{f}$ of skyscraper sheaves $E_{v}$ placed in degree 0 . Viewing $E_{v}$ as a $G_{\kappa(v)}$-module, there is an exact sequence

$$
0 \rightarrow \mathbb{Z} \rightarrow \sum_{i \in C_{v}} \operatorname{Ind}_{G_{\kappa(v)_{i}}}^{G_{\kappa(v)}} \mathbb{Z} \rightarrow E_{v} \rightarrow 0,
$$

where $C_{v}$ is the set of irreducible components of the fibre $\mathcal{X}_{\kappa(v)}$ and $\kappa(v)_{i}$ denotes the algebraic closure of $\kappa(v)$ in the function field of the component $\mathcal{X}_{\kappa(v), i}$ indexed by $i \in C_{v}$. By $[14,(4.25)]$, we have

$$
H^{1}\left(\bar{S}, E_{v}\right) \cong H^{1}\left(S_{v}, E_{v}\right) \cong \mathbb{Z} / \operatorname{gcd}\left(r_{v, i} d_{v, i}\right) \mathbb{Z},
$$

where $r_{v, i}=\left[\kappa(v)_{i}: \kappa(v)\right]$ and $d_{v, i}$ is the multiplicity of $\mathcal{X}_{\kappa(v), i}$ in the fibre. By [3, Remark 1.4] and [4, Theorem 3.1], we have

$$
\operatorname{gcd}\left(r_{v, i} d_{v, i}\right)=\delta_{v} .
$$


The middle row in diagram (71), the fact that $\eta_{*} \eta^{*} P^{\bar{S}}$ is concentrated in degree 0 and Lemma 11 imply $\mathcal{H}^{i}(E)=0$ for $i \geq 2$ and an exact sequence

$$
0 \rightarrow \mathcal{H}^{0}(E) \rightarrow \mathcal{H}^{0}\left(P^{\bar{S}}\right) \rightarrow \eta_{*} \eta^{*} P^{\bar{S}} \rightarrow \mathcal{H}^{1}(E) \rightarrow 0
$$

We saw already that the restriction of $\mathcal{H}^{1}(E)$ to $S$ is 0 , and it remains to show $u_{\infty}^{S, *} \mathcal{H}^{0}(E)=0$ and to compute $u_{\infty}^{S, *} \mathcal{H}^{1}(E)$. If

$$
\operatorname{Spec}(F) \stackrel{\tilde{\eta}}{\rightarrow} U \stackrel{j}{\rightarrow} S \stackrel{\phi^{S}}{\rightarrow} \bar{S}
$$

is an open subscheme over which $f$ is smooth, we have $\tilde{\eta}_{*} \tilde{\eta}^{*} P=P$ and hence

$$
u_{\infty}^{S, *} \eta_{*} \eta^{*} P^{\bar{S}}=u_{\infty}^{S, *} \phi_{*}^{S} j_{*} P=u_{\infty}^{S, *} \phi_{*}^{S} P .
$$

The bottom triangle in diagram (47) and Lemma 11 give an exact sequence

$$
0 \rightarrow \mathcal{H}^{0}\left(P^{\bar{S}}\right) \rightarrow \phi_{*}^{S} P \rightarrow u_{\infty, *}^{S} \mathcal{H}^{0}(K) \rightarrow 0
$$

Applying $u_{\infty}^{S, *}$ to both formulas (75) and (77) and using equation (76), we find $u_{\infty}^{S, *} \mathcal{H}^{0}(E)=0$ and an isomorphism

$$
u_{\infty}^{S, *} \mathcal{H}^{1}(E) \cong \mathcal{H}^{0}(K) \cong \operatorname{ker}\left(\bigoplus_{v \in S_{\infty}} \operatorname{Br}\left(F_{v}\right) \rightarrow \bigoplus_{v \in S_{\infty}} \operatorname{Br}\left(\mathcal{X}_{F_{v}}\right)\right) \cong \bigoplus_{v \in S_{\infty}} \mathbb{Z} / \delta_{v} \mathbb{Z}
$$

The last identity follows from [28, Equation 8] and the exact sequence

$$
\operatorname{Pic}\left(\mathcal{X}_{\bar{F}_{v}}\right)^{G_{F_{v}}} \stackrel{\phi_{v}}{\longrightarrow} \operatorname{Br}\left(F_{v}\right) \rightarrow \operatorname{Br}\left(\mathcal{X}_{F_{v}}\right)
$$

induced by the Hochschild-Serre spectral sequence of the covering $\mathcal{X}_{\bar{F}_{v}} \rightarrow \mathcal{X}_{F_{v}}$ for the sheaf $\mathbb{G}_{m}$.

Lemma 11. For $i \geq 1$ we have

$$
\mathcal{H}^{i}\left(P^{\bar{S}}\right)=0
$$

Proof. Using the long exact sequence induced by the left column in diagram (47) and the fact that $\mathbb{Z}(1)^{\bar{S}}=\phi_{*}^{S} \mathbb{G}_{m}[-1]$ is concentrated in degree 1 [6, Proposition 6.11], it suffices to show

$$
\mathcal{H}^{i}\left(R \bar{f}_{*} \mathbb{Z}(1)^{\overline{\mathcal{X}}}\right)=0, \quad i \geq 3
$$

Using the long exact sequence induced by the central row in diagram (47), it suffices to show that

$$
R \phi_{*}^{S} R f_{*} \mathbb{Z}(1) \rightarrow u_{\infty, *}^{S} R f_{\infty, *} \tau^{>1} R \hat{\pi}_{*}(2 \pi i \mathbb{Z})
$$


is a surjection in degree 2 (this is clear, as the target is zero) and an isomorphism in degrees $\geq 3$. Since $R f_{*} \mathbb{Z}(1)$ is concentrated in degrees $\leq 2$, this is equivalent to

$$
\tau^{\geq 3} u_{\infty}^{S, *} R \phi_{*}^{S} R f_{*} \mathbb{Z}(1) \rightarrow R f_{\infty, *} \tau^{>1} R \hat{\pi}_{*}(2 \pi i \mathbb{Z})
$$

being an isomorphism. But map (78) is isomorphic to the map

$$
R \Gamma\left(G_{\mathbb{R}}, \mathcal{X}(\mathbb{C}),(2 \pi i) \mathbb{Z}\right) \rightarrow R \Gamma\left(\mathcal{X}(\mathbb{R}), \tau^{>1} R \hat{\pi}_{*}(2 \pi i) \mathbb{Z}\right)
$$

in triangle (60), which we have shown to be an isomorphism in degrees $\geq 3$ in the proof of Lemma 9. To see that the two maps are isomorphic in degrees $\geq 3$, use the exact triangles

$$
R f_{\mathbb{C}, *}(2 \pi i) \mathbb{Z} \rightarrow R f_{\mathbb{C}, *}(2 \pi i) \mathbb{Q} \rightarrow R f_{\mathbb{C}, *}(2 \pi i) \mathbb{Q} / \mathbb{Z}
$$

and

$$
R f_{*} \mathbb{Z}(1) \rightarrow R f_{*} \mathbb{Q}(1) \rightarrow R f_{*} \mathbb{Q} / \mathbb{Z}(1)
$$

and the isomorphism $\alpha^{S, *} R f_{*} \mathbb{Q} / \mathbb{Z}(1) \cong R f_{\mathbb{C}, *}(2 \pi i) \mathbb{Q} / \mathbb{Z}$ arising from proper base change

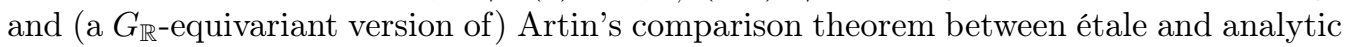
cohomology, where $\alpha^{S}$ is the composite morphism of topoi

$$
S h\left(G_{\mathbb{R}}, S(\mathbb{C})\right) \rightarrow S_{\mathbb{R}, e t} \rightarrow S_{\text {et }}
$$

By [6, Lemma 6.2] we have $u_{\infty}^{S, *} R \phi_{*}^{S}=R \pi_{*}^{S} \alpha^{S, *}$, and hence we find

$$
u_{\infty}^{S, *} R \phi_{*}^{S} R f_{*} \mathbb{Q} / \mathbb{Z}(1) \cong R \Gamma\left(G_{\mathbb{R}}, \mathcal{X}(\mathbb{C}),(2 \pi i) \mathbb{Q} / \mathbb{Z}\right) .
$$

The long exact sequences induced by these two triangles then show that

$$
\mathcal{H}^{i}\left(u_{\infty}^{S, *} R \phi_{*}^{S} R f_{*} \mathbb{Z}(1)\right) \cong H^{i}\left(G_{\mathbb{R}}, \mathcal{X}(\mathbb{C}),(2 \pi i) \mathbb{Z}\right)
$$

for $i \geq 3$, where for $i=3$ we also need the fact that

$$
\mathcal{H}^{2}\left(u_{\infty}^{S, *} R \phi_{*}^{S} R f_{*} \mathbb{Q}(1)\right) \rightarrow H^{2}\left(G_{\mathbb{R}}, \mathcal{X}(\mathbb{C}),(2 \pi i) \mathbb{Q}\right) \cong \mathbb{Q}
$$

is surjective. This is clear, since this map is just the degree map.

We continue with the proof of Proposition 5.1. Taking cohomology over $\bar{S}$ and $\bar{S}_{v}$ of the top row in diagram (71) gives a map of long exact sequences

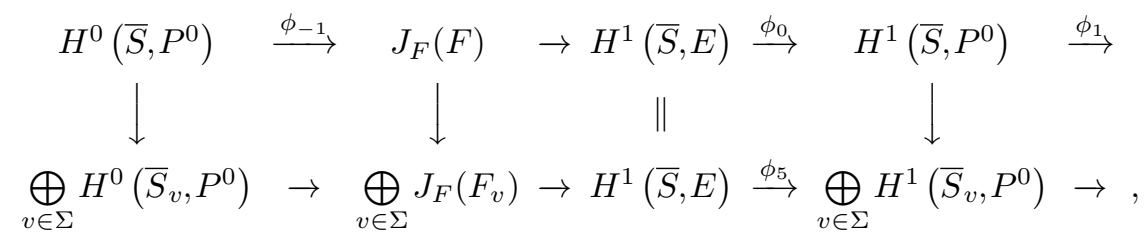


where the vertical isomorphism holds because $E$ is a sum of skyscraper sheaves supported in $\Sigma$. From Lemma 8 and expressions (72) and (69), we have

$$
\# \operatorname{Br}(\overline{\mathcal{X}}) \delta^{2}=\# H^{1}\left(\bar{S}, P^{0}\right)=\frac{\# H^{1}(\bar{S}, E)}{\# \operatorname{coker}\left(\phi_{-1}\right)} \cdot \# \operatorname{im}\left(\phi_{1}\right)=\frac{\prod_{v \in \Sigma} \delta_{v}}{\alpha} \cdot \# \operatorname{im}\left(\phi_{1}\right)
$$

To compute $\# \operatorname{im}\left(\phi_{1}\right)$, consider the continuation of the long exact sequences

$$
\begin{aligned}
& H^{1}(\bar{S}, E) \stackrel{\phi_{0}}{\longrightarrow} \quad H^{1}\left(\bar{S}, P^{0}\right) \quad \stackrel{\phi_{1}}{\longrightarrow} \quad H^{1}\left(\bar{S}, \tilde{P}^{0}\right) \quad \rightarrow H^{2}(\bar{S}, E) \\
& H^{1}(\bar{S}, E) \stackrel{\phi_{5}}{\longrightarrow} \bigoplus_{v \in \Sigma} H^{1}\left(\bar{S}_{v}, P^{0}\right) \stackrel{\phi_{4}}{\longrightarrow} \bigoplus_{v \in \Sigma} H^{1}\left(\bar{S}_{v}, \tilde{P}^{0}\right) \rightarrow H^{2}(\bar{S}, E) \\
& \downarrow \\
& H^{2}\left(\bar{S}, j_{!} j^{*} P^{0}\right) \\
& \downarrow \phi_{6} \\
& H^{2}\left(\bar{S}, P^{0}\right) \text {, }
\end{aligned}
$$

where the vertical column is also exact. Here $j$ is the open immersion $\bar{S} \backslash \Sigma \stackrel{j}{\rightarrow} \bar{S}$. Since $\operatorname{ker}\left(\phi_{2}\right) \subseteq \operatorname{im}\left(\phi_{1}\right)$, we have

$$
\# \operatorname{im}\left(\phi_{1}\right)=\# \operatorname{im}\left(\phi_{2} \circ \phi_{1}\right) \cdot \# \operatorname{ker}\left(\phi_{2}\right)=\# \operatorname{im}\left(\phi_{4} \circ \phi_{3}\right) \cdot \# \operatorname{ker}\left(\phi_{2}\right)
$$

and since $\operatorname{im}\left(\phi_{5}\right) \subseteq \operatorname{im}\left(\phi_{3}\right)$, we obtain

$$
\begin{aligned}
\# \operatorname{im}\left(\phi_{1}\right) & =\# \operatorname{im}\left(\phi_{4} \circ \phi_{3}\right) \cdot \# \operatorname{ker}\left(\phi_{2}\right)=\frac{\# \operatorname{im}\left(\phi_{3}\right)}{\# \operatorname{im}\left(\phi_{5}\right)} \cdot \# \operatorname{ker}\left(\phi_{2}\right) \\
& =\frac{\prod_{v \in \Sigma} \# H^{1}\left(\bar{S}_{v}, P^{0}\right)}{\# \operatorname{ker}\left(\phi_{6}\right) \# \operatorname{im}\left(\phi_{5}\right)} \cdot \# \operatorname{ker}\left(\phi_{2}\right) \\
& =\frac{\prod_{v \in \Sigma} \delta_{v}}{\alpha \prod_{v \in \Sigma}\left(\delta_{v} / \delta_{v}^{\prime}\right)} \cdot \# \amalg\left(J_{F}\right)
\end{aligned}
$$

using Lemmas 12, 13 and 14. Combining equations (79) and (80) finishes the proof.

Lemma 12. For $v \in \Sigma$ we have

$$
H^{1}\left(\bar{S}_{v}, P^{0}\right) \cong \mathbb{Z} / \delta_{v} \mathbb{Z}
$$

and $\operatorname{im}\left(\phi_{5, v}\right) \cong \mathbb{Z} /\left(\delta_{v} / \delta_{v}^{\prime}\right) \mathbb{Z}$, where $\phi_{5}=\oplus_{v} \phi_{5, v}$. 
Proof. Consider the following commutative diagram with exact rows and columns induced by diagram (71):

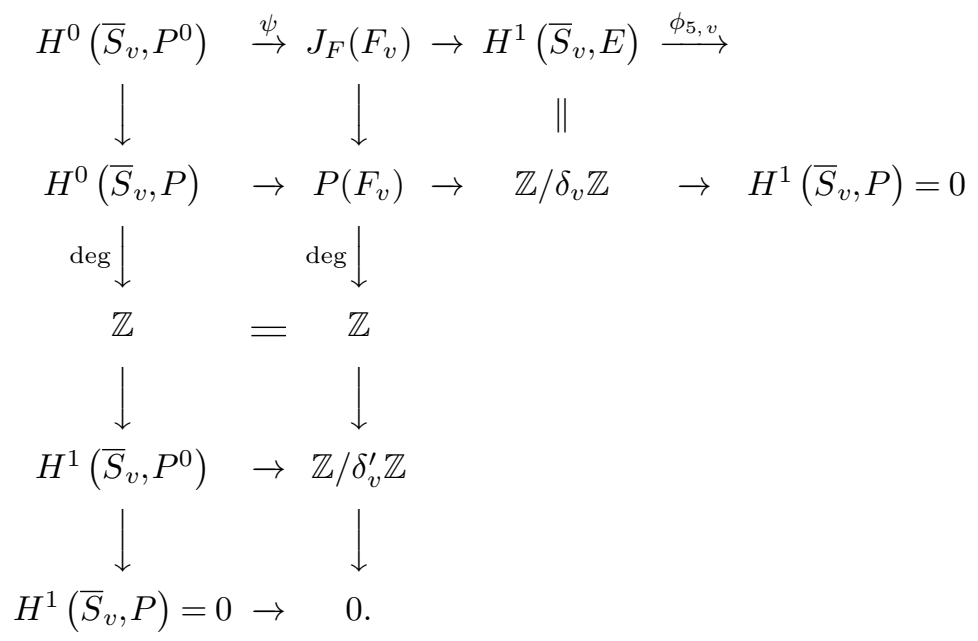

The vanishing of $H^{1}\left(\bar{S}_{v}, P\right)$ is proven in [14, (4.15)] for $v \in \Sigma_{f}$ and follows from Lemma 11 for $v \in S_{\infty}$. The image of $H^{0}\left(\bar{S}_{v}, P\right)$ in $P\left(F_{v}\right)$ coincides with $\operatorname{Pic}\left(\mathcal{X}_{F_{v}}\right)$. For $v \in \Sigma_{f}$ this is because $H^{0}\left(\bar{S}_{v}, P\right)=\operatorname{Pic}\left(\mathcal{X}_{S_{v}}\right) \rightarrow \operatorname{Pic}\left(\mathcal{X}_{F_{v}}\right)$ is surjective, and for $v \in S_{\infty}$ both subgroups coincide with the kernel of the map $P\left(F_{v}\right) \rightarrow \operatorname{Br}\left(F_{v}\right)$. Hence the degree map on $H^{0}\left(\bar{S}_{v}, P\right)$ has image $\delta_{v} \mathbb{Z}$, which gives formula (81). By the snake lemma, we find $\operatorname{coker}(\psi) \cong \mathbb{Z} / \delta_{v}^{\prime} \mathbb{Z}$ and $\operatorname{im}\left(\phi_{5, v}\right) \cong \mathbb{Z} /\left(\delta_{v} / \delta_{v}^{\prime}\right) \mathbb{Z}$.

Lemma 13. We have $\# \operatorname{ker}\left(\phi_{6}\right)=\alpha$.

Proof. For the open subscheme $U:=S \backslash \Sigma_{f}$ of $S$ and any prime $p$, the proof of Proposition 3.1 generalises to prove a duality isomorphism $\mathbf{A V}\left(\mathcal{X}_{U}, 1\right)$ fitting into a commutative diagram of isomorphisms

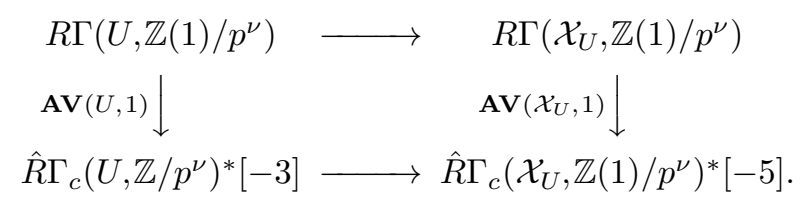

We then obtain diagram (52) with $S$ (resp., $\mathcal{X}$ ) replaced by $U$ (resp., $\mathcal{X}_{U}$ ). Triangle (54) and the left-hand column in diagram (50) then induce an isomorphism $\mathbf{A V}\left(U, P^{0}\right)$ fitting into a commutative diagram 


$$
\begin{array}{ccc}
R \Gamma\left(\bar{S}, P^{0} / p^{\nu}\right) & \longrightarrow \quad R \Gamma\left(U, P^{0} / p^{\nu}\right) \\
\mathbf{A V}\left(\bar{S}, P^{0}\right) \downarrow & \mathbf{A V}\left(U, P^{0}\right) \downarrow \\
R \Gamma\left(\bar{S}, P^{0} / p^{\nu}\right)^{*}[-1] & \longrightarrow \hat{R} \Gamma_{c}\left(U, P^{0} / p^{\nu}\right)^{*}[-1] .
\end{array}
$$

We have isomorphisms

$$
H^{2}\left(\bar{S}, j_{!} P^{0}\right) \cong \hat{H}_{c}^{2}\left(U, P^{0}\right) \cong \hat{H}_{c}^{1}\left(U, P^{0} \otimes \mathbb{Q} / \mathbb{Z}\right) \cong H^{0}\left(U, P^{0}\right)^{*}
$$

where the first isomorphism holds because Tate cohomology agrees with ordinary cohomology in degrees $\geq 1$, the second because $\hat{H}_{c}^{i}\left(U, P^{0}\right)$ is torsion for $i=1,2$ and the third by taking the limit of $\mathbf{A V}\left(U, P^{0}\right)^{*}$ over all $p$ and all $\nu$. We find that $\phi_{6}$ is dual to the natural restriction map

$$
\phi_{6}^{*}: H^{0}\left(\bar{S}, P^{0}\right) \cong \operatorname{Pic}^{0}(\mathcal{X}) / \operatorname{Pic}\left(\mathcal{O}_{F}\right) \rightarrow H^{0}\left(U, P^{0}\right) \cong J_{F}(F),
$$

where the last isomorphism holds because $f$ is smooth over $U$, and hence $P^{0}$ coincides with the (sheaf represented by the) Néron model of $J_{F}$. From definition (69) of $\alpha$ we conclude that $\alpha=\# \operatorname{coker}\left(\phi_{6}^{*}\right)=\# \operatorname{ker}\left(\phi_{6}\right)$.

Lemma 14. There is an isomorphism $\operatorname{ker}\left(\phi_{2}\right) \cong \amalg\left(J_{F}\right)$.

Proof. Consider the following commutative diagram with exact rows and columns:

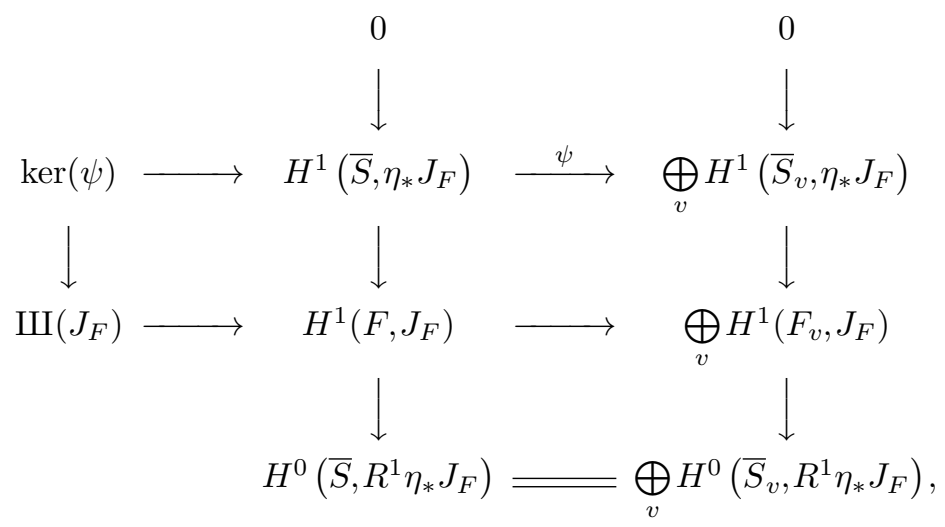

where the vertical exact sequences arise from the Leray spectral sequence for the morphism $\eta$. Note that $R^{1} \eta_{*} J_{F}$ is a sum of skyscraper sheaves (with stalk $H^{1}\left(I_{v}, J_{F}\right)$ ), hence the bottom identity. An easy diagram chase then shows that $\operatorname{ker}(\psi) \cong \amalg\left(J_{F}\right)$. Exactly the same argument applies to the diagram 


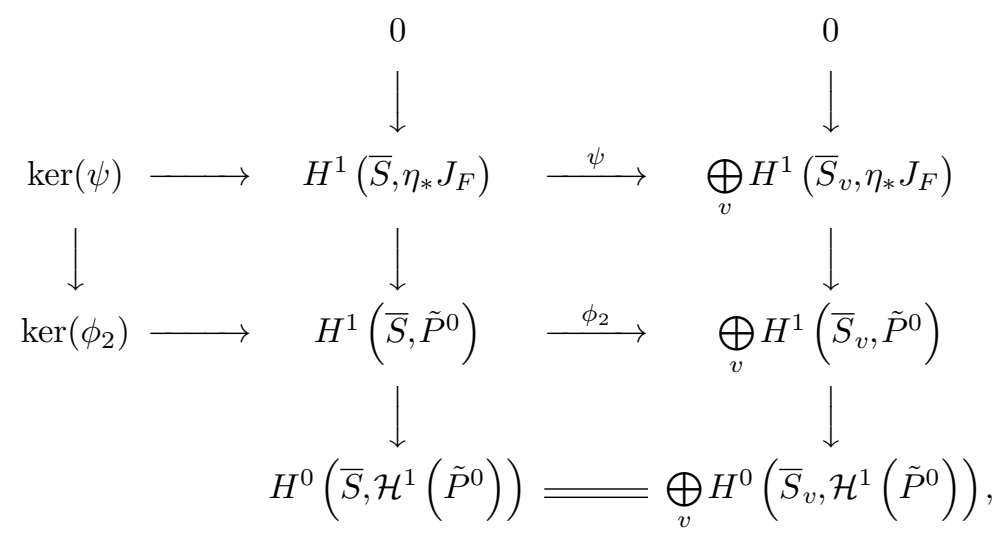

where now the vertical maps arise from the hypercohomology spectral sequence for the complex $\tilde{P}^{0}$, noting that $\mathcal{H}^{0}\left(\tilde{P}^{0}\right) \cong \eta_{*} J_{F}$ and that $\mathcal{H}^{1}\left(\tilde{P}^{0}\right)$ is a sum of skyscraper sheaves over points in $\Sigma$. This finishes the proof.

\section{Comparison to the Birch and Swinnerton-Dyer conjecture}

We begin with a few auxiliary lemmas and then state and prove our main comparison result, Theorem 6.1 .

By an inner product on a finitely generated abelian group $N$ we mean a (either positive or negative) definite, symmetric bilinear form $\langle-,-\rangle$ on $N_{\mathbb{R}}:=N \otimes_{\mathbb{Z}} \mathbb{R}$. For such an $N$ we define

$$
\Delta(N):=\frac{\left|\operatorname{det}\left(\left\langle b_{i}, b_{j}\right\rangle\right)\right|}{\left[N: \bigoplus_{i} \mathbb{Z} b_{i}\right]^{2}},
$$

where $\left\{b_{i}\right\} \subseteq N$ is a maximal linearly independent subset. It is easily verifiable that $\Delta(N)$ depends only on $\langle-,-\rangle$, not on the choice of $\left\{b_{i}\right\}$.

Lemma 15. Let

$$
\cdots \rightarrow N_{i} \stackrel{d_{i}}{\rightarrow} N_{i+1} \rightarrow \cdots
$$

be an exact sequence of finitely generated abelian groups of finite length. Assume each $N_{i}$ is equipped with an inner product $\tau_{i}$ so that

$$
\left.\tau_{i+1}\right|_{\operatorname{im}\left(d_{i}\right)_{\mathbb{R}}}=\left.\tau_{i}\right|_{\operatorname{ker}\left(d_{i}\right)_{\mathbb{R}}^{\perp}}
$$

via the isomorphism $d_{i}: \operatorname{ker}\left(d_{i}\right)_{\mathbb{R}}^{\perp} \cong \operatorname{im}\left(d_{i}\right)_{\mathbb{R}}$ induced by $d_{i}$. Then

$$
\prod_{i} \Delta\left(N_{i}\right)^{(-1)^{i}}=1
$$

Proof. It suffices to prove the statement for the short exact sequences

$$
0 \rightarrow \operatorname{ker}\left(d_{i}\right) \rightarrow N_{i} \rightarrow \operatorname{im}\left(d_{i}\right) \rightarrow 0 .
$$


A maximal linearly independent subset $\left\{b_{j}\right\}_{1 \leq j \leq l} \subset \operatorname{ker}\left(d_{i}\right)$ can be extended to a maximal linearly independent subset $\left\{b_{j}\right\}_{1 \leq j \leq l+k} \subset N_{i}$, and $\left\{\bar{b}_{j}\right\}_{l+1 \leq j \leq l+k} \subset \operatorname{im}\left(d_{i}\right)$ is then also a maximal linearly independent subset. By the snake lemma we have

$$
\left[N_{i}: \bigoplus_{j=1}^{l+k} \mathbb{Z} b_{j}\right]=\left[\operatorname{ker}\left(d_{i}\right): \bigoplus_{j=1}^{l} \mathbb{Z} b_{j}\right] \cdot\left[\operatorname{im}\left(d_{i}\right): \bigoplus_{j=l+1}^{l+k} \mathbb{Z} \bar{b}_{j}\right],
$$

and we also have

$$
\operatorname{det}\left(\left\langle b_{j}, b_{j^{\prime}}\right\rangle\right)_{1 \leq j, j^{\prime} \leq l+k}=\operatorname{det}\left(\left\langle b_{j}, b_{j^{\prime}}\right\rangle\right)_{1 \leq j, j^{\prime} \leq l} \cdot \operatorname{det}\left(\left\langle\bar{b}_{j}, \bar{b}_{j^{\prime}}\right\rangle\right)_{l+1 \leq j, j^{\prime} \leq l+k},
$$

since the $\left\{b_{j}\right\}_{l+1 \leq j \leq l+k}$ can be modified into elements of $\operatorname{ker}\left(d_{i}\right)_{\mathbb{R}}^{\perp}$ (by adding elements of $\left.\operatorname{ker}\left(d_{i}\right)_{\mathbb{R}}\right)$ without changing $\operatorname{det}\left(\left\langle\bar{b}_{j}, \bar{b}_{j^{\prime}}\right\rangle\right)_{l+1 \leq j, j^{\prime} \leq l+k}$ or $\operatorname{det}\left(\left\langle b_{j}, b_{j^{\prime}}\right\rangle\right)_{1 \leq j, j^{\prime} \leq l+k}$.

Remark 3. If all the groups $N_{i}$ are torsion free, Lemma 15 is a well-known statement in Arakelov theory. It amounts to additivity of the first Chern class of Arakelov vector bundles on $\operatorname{Spec}(\mathbb{Z})$ [32, III.4.3].

Recall that for each $v \in \Sigma_{f}$, we denote by $C_{v}$ the set of irreducible components of the fibre $\mathcal{X}_{\kappa(v)}$ and by $r_{v, i}=\left[\kappa(v)_{i}: \kappa(v)\right]$ the degree of the constant field of the component corresponding to $i \in C_{v}$.

Lemma 16. Let $L\left(J_{F}, s\right)$ be the Hasse-Weil L-function of the Jacobian of $\mathcal{X}_{F}$. Then

$$
\operatorname{ord}_{s=1} \zeta\left(H^{1}, s\right)=\operatorname{ord}_{s=1} L\left(J_{F}, s\right)+\sum_{v \in \Sigma_{f}}\left(\# C_{v}-1\right)
$$

and

$$
\zeta^{*}\left(H^{1}, 1\right)=L^{*}\left(J_{F}, 1\right) \cdot \prod_{v \in \Sigma_{f}}(\log N v)^{\# C_{v}-1} \prod_{i \in C_{v}} r_{v, i}
$$

Proof. Denoting by $Y_{0}$ the set of closed points of a scheme $Y$, we have

$$
\begin{aligned}
\zeta(\mathcal{X}, s) & =\prod_{x \in \mathcal{X}_{0}} \frac{1}{1-\# \kappa(x)^{-s}}=\prod_{v \in S_{0}} \zeta\left(\mathcal{X}_{\kappa(v)}, s\right) \\
& =\prod_{v \in S_{0}} \prod_{m=0}^{2} \operatorname{det}_{\mathbb{Q}_{l}}\left(\mathrm{id}-N v^{-s} \operatorname{Fr}_{v} \mid H^{m}\left(\mathcal{X}_{\overline{\kappa(v)}}, \mathbb{Q}_{l}\right)\right)^{(-1)^{m+1}}
\end{aligned}
$$

and isomorphisms [2, Lemma 1.2]

$$
\begin{aligned}
& H^{0}\left(\mathcal{X}_{\overline{\kappa(v)}}, \mathbb{Q}_{l}\right) \cong H^{0}\left(\mathcal{X}_{\bar{F}}, \mathbb{Q}_{l}\right)^{I_{v}} \cong \mathbb{Q}_{l} \\
& H^{1}\left(\mathcal{X}_{\overline{\kappa(v)}}, \mathbb{Q}_{l}\right) \cong H^{1}\left(\mathcal{X}_{\bar{F}}, \mathbb{Q}_{l}\right)^{I_{v}}
\end{aligned}
$$

and an exact sequence

$$
0 \rightarrow E_{v} \otimes_{\mathbb{Z}} \mathbb{Q}_{l}(-1) \rightarrow H^{2}\left(\mathcal{X}_{\overline{\kappa(v)}}, \mathbb{Q}_{l}\right) \rightarrow H^{2}\left(\mathcal{X}_{\bar{F}}, \mathbb{Q}_{l}\right)^{I_{v}} \cong \mathbb{Q}_{l}(-1) \rightarrow 0
$$


From sequence (73), we have

$$
\operatorname{det}_{\mathbb{Q}_{l}}\left(\mathrm{id}-T \cdot \operatorname{Fr}_{v} \mid E_{v} \otimes_{\mathbb{Z}} \mathbb{Q}_{l}(-1)\right)=\frac{1}{1-T \cdot N v} \prod_{i \in C_{v}}\left(1-(T \cdot N v)^{r_{v, i}}\right) .
$$

We then define

$$
\begin{aligned}
& \zeta\left(H^{0}, s\right):=\prod_{v \in S_{0}} \operatorname{det}_{\mathbb{Q}_{l}}\left(\operatorname{id}-N v^{-s} \operatorname{Fr}_{v} \mid \mathbb{Q}_{l}\right)^{-1}=\zeta_{F}(s) \\
& \zeta\left(H^{2}, s\right):=\prod_{v \in S_{0}} \operatorname{det}_{\mathbb{Q}_{l}}\left(\mathrm{id}-N v^{-s} \operatorname{Fr}_{v} \mid \mathbb{Q}_{l}(-1)\right)^{-1}=\zeta_{F}(s-1)
\end{aligned}
$$

and

$$
\begin{aligned}
\zeta\left(H^{1}, s\right):= & \prod_{v \in S_{0}} \operatorname{det}_{\mathbb{Q}_{l}}\left(\mathrm{id}-N v^{-s} \operatorname{Fr}_{v} \mid H^{1}\left(\mathcal{X}_{\bar{F}}, \mathbb{Q}_{l}\right)^{I_{v}}\right)^{-1} \\
& \cdot \prod_{v \in \Sigma_{f}} \operatorname{det}_{\mathbb{Q}_{l}}\left(\mathrm{id}-N v^{-s} \operatorname{Fr}_{v} \mid E_{v} \otimes_{\mathbb{Z}} \mathbb{Q}_{l}(-1)\right) \\
= & L\left(J_{F}, s\right) \cdot \prod_{v \in \Sigma_{f}} \frac{1}{1-N v^{-(s-1)}} \prod_{i \in C_{v}}\left(1-N v^{-(s-1) r_{v, i}}\right) .
\end{aligned}
$$

From this equations (83) and (84) are immediate. Comparing the definition of $\zeta\left(H^{i}, s\right)$ with equation (85), we deduce the factorisation (4). For connections with the perverse $t$-structure on $l$-adic sheaves we refer to [6, Remark 7.5] and to Remark 5 .

Lemma 17. For each $v \in \Sigma_{f}$, we have

$$
\Delta\left(E_{v}^{G_{\kappa(v)}}\right)=\frac{\# \Phi_{v}}{\delta_{v}^{\prime} \delta_{v}}(\log N v)^{\# C_{v}-1} \prod_{i \in C_{v}} r_{v, i}
$$

where $\Phi_{v}$ is the component group of the Néron model of $J_{F}$ at $v$. Here $\Delta$ is formed with respect to the Arakelov intersection pairing.

Proof. Recall that we denote by $d_{v, i}$ the multiplicity of the irreducible component $\mathcal{X}_{\kappa(v), i}$ in the fibre $\mathcal{X}_{\kappa(v)}$. Let $\mathbb{Z}^{C_{v}}$ be the group of divisors on $\mathcal{X}$ supported in $\mathcal{X}_{\kappa(v)}$, and denote by $\left\langle D_{1}, D_{2}\right\rangle=\left.\operatorname{deg} \mathcal{O}\left(D_{1}\right)\right|_{D_{2}}$ the intersection number of two divisors.

Taking $G_{\kappa(v)}$-cohomology of sequence (73), we obtain a short exact sequence

$$
0 \rightarrow \mathbb{Z} \stackrel{\gamma}{\rightarrow} \mathbb{Z}^{C_{v}} \rightarrow E_{v}^{G_{\kappa(v)}} \rightarrow H^{1}\left(G_{\kappa(v)}, \mathbb{Z}\right)=0,
$$

where $\gamma: 1 \mapsto \mathcal{X}_{\kappa(v)}=\sum_{i} d_{v, i} \mathcal{X}_{\kappa(v), i}$ by [14, (4.22)]. So $\operatorname{im}(\gamma)$ lies in the image of $\operatorname{Pic}\left(\mathcal{O}_{F}\right)$, and $\tilde{E}_{v}:=E_{v}^{G_{\kappa(v)}}$ is the subgroup of $\operatorname{Pic}^{0}(\mathcal{X}) / \operatorname{Pic}\left(\mathcal{O}_{F}\right)$ generated by divisors supported in $\mathcal{X}_{\kappa(v)}$. Denoting by $N^{\star}=\operatorname{Hom}_{\mathbb{Z}}(N, \mathbb{Z})$ the $\mathbb{Z}$-dual, define a complex

$$
\mathbb{Z}^{C_{v}} \stackrel{\tilde{\alpha}}{\rightarrow} \mathbb{Z}^{C_{v}} \cong\left(\mathbb{Z}^{C_{v}}\right)^{\star} \stackrel{\gamma^{\star}}{\longrightarrow} \mathbb{Z}^{\star} \cong \mathbb{Z},
$$


where

$$
\tilde{\alpha}(D)=\sum_{i}\left\langle D, \mathcal{X}_{\kappa(v), i}\right\rangle \mathcal{X}_{\kappa(v), i}
$$

Since the intersection pairing is trivial on the image of $\operatorname{Pic}\left(\mathcal{O}_{F}\right)$, the map $\tilde{\alpha}$ factors through $\tilde{E}_{v}$ and

$$
\operatorname{ker}\left(\gamma^{\star}\right) / \operatorname{im}(\tilde{\alpha}) \cong \tilde{E}_{v}^{\star} / \tilde{\alpha}\left(\tilde{E}_{v}\right)
$$

has cardinality $\left|\operatorname{det}\left(\left\langle b_{i}, b_{j}\right\rangle\right)\right|$, where $\left\{b_{i}\right\}$ is a $\mathbb{Z}$-basis of $\tilde{E}_{v} /\left(\tilde{E}_{v}\right)_{\text {tor }}$. Since

$$
\left(\tilde{E}_{v}\right)_{t o r} \cong \mathbb{Z} / d_{v} \mathbb{Z}, \quad d_{v}:=\operatorname{gcd}\left(d_{v, i}\right)
$$

and since the Arakelov intersection pairing is given by $\langle-,-\rangle \log N v$ [19], we find

$$
\frac{\Delta\left(E_{v}^{G_{\kappa(v)}}\right)}{(\log N v)^{\# C_{v}-1}}=\frac{\Delta\left(\tilde{E}_{v}\right)}{(\log N v)^{\# C_{v}-1}}=\frac{\left|\operatorname{det}\left(\left\langle b_{i}, b_{j}\right\rangle\right)\right|}{d_{v}^{2}}=\frac{\# \operatorname{ker}\left(\gamma^{\star}\right) / \operatorname{im}(\tilde{\alpha})}{d_{v}^{2}} .
$$

On the other hand, by [3, Theorem 1.11] and [23, proof of Lemma 4.4] there is an exact sequence

$$
0 \rightarrow \operatorname{ker}(\beta) / \operatorname{im}(\alpha) \rightarrow \Phi_{v} \rightarrow \mathbb{Z} /\left(\delta_{v}^{\prime} / d_{v}\right) \mathbb{Z} \rightarrow 0
$$

where

$$
\mathbb{Z}^{C_{v}} \stackrel{\alpha}{\rightarrow} \mathbb{Z}^{C_{v}} \stackrel{\beta}{\rightarrow} \mathbb{Z}
$$

are the maps

$$
\alpha(D)=\sum_{i} r_{v, i}^{-1}\left\langle D, \mathcal{X}_{\kappa(v), i}\right\rangle \mathcal{X}_{\kappa(v), i}, \quad \beta\left(\mathcal{X}_{\kappa(v), i}\right)=r_{v, i} d_{v, i}
$$

We have $\tilde{\alpha}=r \alpha$, where $r: \mathbb{Z}^{C_{v}} \rightarrow \mathbb{Z}^{C_{v}}$ is the map $\left(n_{i}\right) \mapsto\left(r_{v, i} n_{i}\right)$. The snake lemma applied to

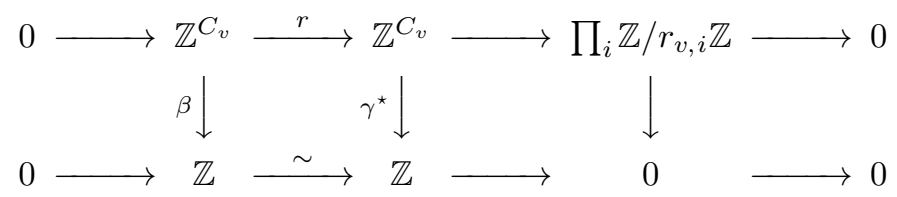

gives an exact sequence

$$
0 \rightarrow \operatorname{ker}(\beta) \stackrel{r}{\rightarrow} \operatorname{ker}\left(\gamma^{*}\right) \rightarrow R \rightarrow 0
$$

where

$$
\# R=\frac{\left(\prod_{i} r_{v, i}\right) \# \operatorname{coker}\left(\gamma^{*}\right)}{\# \operatorname{coker}(\beta)}=\frac{\left(\prod_{i} r_{v, i}\right) d_{v}}{\operatorname{gcd}\left(r_{v, i} d_{v, i}\right)}=\frac{\prod_{i} r_{v, i}}{\left(\delta_{v} / d_{v}\right)}
$$

using equation (74). Hence,

$$
\# \operatorname{ker}\left(\gamma^{\star}\right) / \operatorname{im}(\tilde{\alpha})=\# \operatorname{ker}\left(\gamma^{\star}\right) / r(\operatorname{im}(\alpha))=\# R \cdot \# \operatorname{ker}(\beta) / \operatorname{im}(\alpha) .
$$

Combining formulas (86) through (89) finishes the proof. 
For the next lemma, recall that for any scheme $Y$ we denote by $Y(\mathbb{C})$ the set of complex points - that is, scheme morphisms $\operatorname{Spec}(\mathbb{C}) \rightarrow Y$ - with its natural $G_{\mathbb{R}}$-action. For a ring $A$ we defin $Y_{A}:=Y \times_{\operatorname{Spec}(\mathbb{Z})} \operatorname{Spec}(A)$, and for a $\mathbb{C}$-scheme $Y \rightarrow \operatorname{Spec}(\mathbb{C})$ we set

$$
Y[\mathbb{C}]=\operatorname{Hom}_{\operatorname{Spec}(\mathbb{C})}(\operatorname{Spec}(\mathbb{C}), Y) .
$$

If $Y \rightarrow \operatorname{Spec}(F)$ is smooth and proper with geometrically connected fibres, then

$$
Y(\mathbb{C})=Y_{\mathbb{C}}[\mathbb{C}]=\coprod_{\tau} Y_{\tau}[\mathbb{C}]
$$

has a natural topology as a disjoint union of connected, compact complex manifolds indexed by the embeddings $\tau: F \rightarrow \mathbb{C}$. Here $Y_{\tau}:=Y \times_{\operatorname{Spec}(F), \tau} \operatorname{Spec}(\mathbb{C})$. The comparison isomorphism $H^{1}(Y(\mathbb{C}), \mathbb{C}) \simeq H_{d R}^{1}\left(Y_{\mathbb{C}} / \mathbb{C}\right)$ induces an isomorphism of real vector spaces

$$
\begin{aligned}
H^{1}(Y(\mathbb{C}),(2 \pi i) \mathbb{R})^{G_{\mathbb{R}}} & \rightarrow H^{1}(Y(\mathbb{C}), \mathbb{C})^{G_{\mathbb{R}}} \simeq H_{d R}^{1}\left(Y_{\mathbb{C}} / \mathbb{C}\right)^{G_{\mathbb{R}}} \simeq H_{d R}^{1}\left(Y_{\mathbb{R}} / \mathbb{R}\right) \rightarrow \\
& \rightarrow H_{d R}^{1}\left(Y_{\mathbb{R}} / \mathbb{R}\right) / F^{1} \simeq H^{1}\left(Y_{\mathbb{R}}, \mathcal{O}_{Y_{\mathbb{R}}} \simeq H^{1}\left(Y, \mathcal{O}_{Y}\right) \otimes_{\mathbb{Q}} \mathbb{R}\right.
\end{aligned}
$$

which we call the Deligne period isomorphism.

Lemma 18. Let $p: \mathcal{J} \rightarrow \operatorname{Spec}\left(\mathcal{O}_{F}\right)$ be the Néron model of $J_{F}$ and $\operatorname{Lie}(\mathcal{J})$ be its Lie algebra.

(a) $\operatorname{Lie}(\mathcal{J})$ is a projective $\mathcal{O}_{F}$-module of rank $g$ with dual $H^{0}\left(\mathcal{J}, \Omega_{\mathcal{J} / S}\right)$.

(b) There is a natural isomorphism

$$
\operatorname{Lie}(\mathcal{J})_{\mathbb{Q}} \cong H^{1}\left(J_{F}, \mathcal{O}_{J_{F}}\right),
$$

and if $\Omega(\mathcal{J}) \in \mathbb{R}^{\times}$is such that

$$
\operatorname{det}_{\mathbb{Z}} H^{1}\left(J_{F}(\mathbb{C}),(2 \pi i) \mathbb{Z}\right)^{G_{\mathbb{R}}}=\Omega(\mathcal{J}) \cdot \operatorname{det}_{\mathbb{Z}} \operatorname{Lie}(\mathcal{J})
$$

under the Deligne period isomorphism for $Y=J_{F}$, then

$$
\Omega(\mathcal{J})=\Omega(\mathcal{X})
$$

up to sign.

(c) Let

$$
\mathcal{O}_{F} \cdot \omega_{1}+\cdots+\mathcal{O}_{F} \cdot \omega_{g} \subseteq H^{0}\left(\mathcal{J}, \Omega_{\mathcal{J} / S}\right)
$$

be a free submodule of rank $g$ and index $I \in \mathbb{Z}$. If $\mu_{v}$ denotes the Haar measure on $J_{F}\left(F_{v}\right)$ induced by the Lebesgue measure on $F_{v}^{g}$ via the basis $\omega_{i}$, then

$$
\Omega(\mathcal{J})=\frac{1}{I} \cdot\left(2^{-r_{2}} \sqrt{\left|D_{F}\right|}\right)^{-g} \cdot \prod_{v \in S_{\infty}} \mu_{v}\left(J_{F}\left(F_{v}\right)^{0}\right),
$$

where $D_{F}$ is the discriminant of $F$ and $r_{2}$ the number of complex places. Moreover,

$$
\mu_{v}\left(J_{F}\left(F_{v}\right)^{0}\right)= \begin{cases}\int_{J_{F}\left(F_{v}\right)^{0}} \omega_{1} \wedge \cdots \wedge \omega_{g}, & v \text { real }, \\ (2 i)^{-g} \int_{J_{F}\left(F_{v}\right)} \omega_{1} \wedge \cdots \wedge \omega_{g} \wedge \bar{\omega}_{1} \wedge \cdots \wedge \bar{\omega}_{g}, & v \text { complex } .\end{cases}
$$


Proof. By [23, Proposition 1.1b], Lie $(\mathcal{J}) \cong \underline{\operatorname{Hom}}_{S}\left(e^{*} \Omega_{\mathcal{J} / S}, \mathcal{O}_{S}\right)$, where $e: S \rightarrow \mathcal{J}$ is the zero section. Since $p$ is smooth of relative dimension $g, e^{*} \Omega_{\mathcal{J} / S}$ is locally free of rank $g$. From the theory of Néron models,

$$
e^{*} \Omega_{\mathcal{J} / S} \cong p_{*} \Omega_{\mathcal{J} / S}=H^{0}\left(\mathcal{J}, \Omega_{\mathcal{J} / S}\right),
$$

where we identify coherent sheaves on affine schemes with their module of global sections. This gives (a).

For (b), consider the Abel-Jacobi map over $F$

$$
a: \mathcal{X}_{F} \rightarrow J_{F}^{1}
$$

where $J_{F}^{1}$ is the Albanese torsor, a principal homogeneous space under the abelian variety $J_{F}[13$, Theorem 3.3(iii)]. The morphism $a$ induces an isomorphism of motives (systems of realisations, even integrally)

$$
a^{*}: H^{1}\left(J_{F}^{1}\right)(1) \simeq H^{1}\left(\mathcal{X}_{F}\right)(1)
$$

and hence a commutative diagram of Deligne period isomorphisms. In the Betti realisation we obtain an isomorphism of $G_{\mathbb{R}}$-modules

$$
H^{1}(\mathcal{X}(\mathbb{C}),(2 \pi i) \mathbb{Z}) \cong H^{1}\left(J_{F}^{1}(\mathbb{C}),(2 \pi i) \mathbb{Z}\right) \cong H^{1}\left(J_{F}(\mathbb{C}),(2 \pi i) \mathbb{Z}\right) .
$$

Note here that the isomorphism $J_{F}^{1}(\mathbb{C}) \cong J_{F}(\mathbb{C})$ need not be $G_{\mathbb{R}}$-equivariant, but the difference between the actions on both sides is a translation, and translations act trivially on $H^{1}$ of a torus. The Abel-Jacobi map also induces an isomorphism

$$
a^{*}: \operatorname{Pic}_{J_{F} / F}^{0} \cong \operatorname{Pic}_{J_{F}^{1} / F}^{0} \cong \operatorname{Pic}_{\mathcal{X}_{F} / F}^{0}
$$

and hence a commutative diagram of isomorphisms

$$
\begin{gathered}
\operatorname{Lie}\left(\operatorname{Pic}_{J_{F} / F}^{0}\right) \stackrel{\sim}{\longrightarrow} H^{1}\left(J_{F}, \mathcal{O}_{J_{F}}\right) \\
a^{*} \downarrow \sim \\
\operatorname{Lie}(\mathcal{J})_{\mathbb{Q}} \cong \operatorname{Lie}\left(J_{F}\right) \stackrel{\kappa}{\longleftarrow} \operatorname{Lie}\left(\operatorname{Pic}_{\mathcal{X}_{F} / F}^{0}\right) \stackrel{\sim}{\sim} \stackrel{\sim}{\longrightarrow} H^{1}\left(\mathcal{X}_{F}, \mathcal{O}_{\mathcal{X}_{F}}\right),
\end{gathered}
$$

where the computation of Lie algebras is [23, Propositions 1.1d and 1.3b]. The isomorphism $\tau$ is constructed in $[23,1.4]$ using autoduality of the Jacobian, which we have also implicitly used by identifying $J_{F}$ with the Albanese variety of $\mathcal{X}_{F}$. We come to the key result in the proof. By [23, Theorem 3.1], for each finite place $\mathfrak{p}$ of $F$ the natural map (inducing the isomorphism $\kappa$ on the generic fibre)

$$
H^{1}\left(\mathcal{X}_{\mathcal{O}_{F, \mathfrak{p}}}, \mathcal{O}_{\mathcal{X}_{\mathcal{O}_{F, \mathfrak{p}}}}\right) \cong \operatorname{Lie}\left(\operatorname{Pic}_{\mathcal{X}_{\mathcal{O}_{F, p}} / \mathcal{O}_{F, \mathfrak{p}}}\right) \rightarrow \operatorname{Lie}\left(\mathcal{J}_{\mathcal{O}_{F, \mathfrak{p}}}\right)
$$

has kernel and cokernel of the same length over $\mathcal{O}_{F, \mathfrak{p}}$. This implies

$$
\operatorname{det}_{\mathbb{Z}} \operatorname{Lie}(\mathcal{J})=\operatorname{det}_{\mathbb{Z}} H^{1}\left(\mathcal{X}, \mathcal{O}_{\mathcal{X}}\right)
$$

under the determinants of the isomorphisms in diagram (90) and finishes the proof of (b). 
Using the autoduality of $J_{F}$, we obtain a $G_{\mathbb{R}}$-isomorphism

$$
H^{1}\left(J_{F}(\mathbb{C}),(2 \pi i) \mathbb{Z}\right) \cong H_{1}\left(J_{F}(\mathbb{C}), \mathbb{Z}\right)
$$

and also have the isomorphism

$$
H^{1}\left(J_{F}, \Omega_{J_{F}}\right) \cong \operatorname{Lie}\left(J_{F}\right) \cong \operatorname{Hom}_{F}\left(H^{0}\left(J_{F}, \Omega_{J_{F} / F}\right), F\right)
$$

constructed in diagram (90). The Deligne period isomorphism is induced by taking $c$ invariants of the integration pairing

$$
H_{1}\left(J_{F}(\mathbb{C}), \mathbb{Z}\right)_{\mathbb{C}}^{G_{\mathbb{R}}} \times H^{0}\left(J_{F}, \Omega_{J_{F} / F}\right)_{\mathbb{C}} \rightarrow F_{\mathbb{C}} \cong \prod_{\tau} \mathbb{C} \stackrel{\operatorname{Tr}}{\longrightarrow} \mathbb{C}, \quad(\gamma, \omega) \mapsto \int_{\gamma} \omega
$$

where $c$ is complex conjugation on the coefficents $\mathbb{C}$. The trace map $\mathcal{O}_{F} \stackrel{\operatorname{Tr}}{\longrightarrow} \mathbb{Z}$ induces an isomorphism

$$
\operatorname{Lie}(\mathcal{J}) \cong \operatorname{Hom}_{\mathcal{O}_{F}}\left(H^{0}\left(\mathcal{J}, \Omega_{\mathcal{J} / S}\right), \mathcal{O}_{F}\right) \cong \operatorname{Hom}_{\mathbb{Z}}\left(H^{0}\left(\mathcal{J}, \Omega_{\mathcal{J} / S}\right) \otimes_{\mathcal{O}_{F}} \mathcal{D}_{F}^{-1}, \mathbb{Z}\right)
$$

where $\mathcal{D}_{F}^{-1}$ is the inverse different.

For each real (resp., complex) place $v$ of $F$, choose a $\mathbb{Z}$-basis $\gamma_{1}^{v}, \ldots, \gamma_{g}^{v}\left(\right.$ resp., $\left.\gamma_{1}^{v}, \ldots, \gamma_{2 g}^{v}\right)$ of $H_{1}\left(J_{F}\left(F_{v}\right)^{0}, \mathbb{Z}\right)$ so that $\gamma_{i}^{v}$ is a $\mathbb{Z}$-basis of

$$
H_{1}\left(J_{F}(\mathbb{C}), \mathbb{Z}\right)^{G \mathbb{R}} \cong \prod_{v \in S_{\infty}} H_{1}\left(J_{F}\left(F_{v}\right)^{0}, \mathbb{Z}\right)
$$

and let $\beta_{k}$ be a $\mathbb{Z}$-basis of $\mathcal{D}_{F}^{-1}$. Then

$$
\Omega(\mathcal{J})=\frac{1}{I} \cdot \operatorname{det}\left(\int_{\gamma_{i}^{v}} \omega_{j} \otimes \beta_{k}\right)_{(i, v),(j, k)} .
$$

Now let $e_{\tau}$ be the indecomposable idempotents in

$$
\mathcal{D}_{F}^{-1} \otimes_{\mathbb{Z}} \mathbb{C} \cong \mathcal{O}_{F} \otimes_{\mathbb{Z}} \mathbb{C} \cong \prod_{\tau} \mathbb{C}
$$

and express the $\beta_{k}$ as linear combination of the $e_{\tau}$. Then the base change matrix has determinant $\left(\sqrt{D_{F}}\right)^{-1}$ and

$$
\Omega(\mathcal{J})=\frac{1}{I} \cdot\left(\sqrt{D_{F}}\right)^{-g} \cdot \operatorname{det}\left(\int_{\gamma_{i}^{v}} \omega_{j} \otimes e_{\tau}\right)_{(i, v),(j, \tau)}=\frac{1}{I} \cdot\left(\sqrt{D_{F}}\right)^{-g} \cdot \prod_{v \in S_{\infty}} \Omega_{v}
$$

where

$$
\Omega_{v}:=\operatorname{det}\left(\int_{\gamma_{i}^{v}} \omega_{j}^{\tau}\right)_{i,(j, \tau=v)}= \begin{cases}\int_{J_{F}\left(F_{v}\right)^{0}} \omega_{1}^{v} \wedge \cdots \wedge \omega_{g}^{v}, & v \text { real } \\ \int_{J_{F}\left(F_{v}\right)^{0}} \bar{\omega}_{1}^{v} \wedge \cdots \wedge \bar{\omega}_{g}^{v} \wedge \omega_{1}^{v} \wedge \cdots \wedge \omega_{g}^{v}, & v \text { complex. }\end{cases}
$$


Since the Lebesgue measure on $\mathbb{C}$ is induced by the form $d x \wedge d y=(2 i)^{-1} d \bar{z} \wedge d z$, we find

$$
\Omega(\mathcal{J})=\frac{1}{I} \cdot\left(\sqrt{D_{F}}\right)^{-g} \cdot(2 i)^{r_{2} \cdot g} \cdot \prod_{v \in S_{\infty}} \mu_{v}\left(J_{F}\left(F_{v}\right)^{0}\right),
$$

and since $D_{F}$ has sign $(-1)^{r_{2}}$, this concludes the proof of (c).

We now have all the ingredients to compare equations (5) and (6) with the Birch and Swinnerton-Dyer conjecture. We summarise the main result of this paper in the following theorem:

Theorem 6.1. Let $f: \mathcal{X} \rightarrow S$ be an arithmetic surface as in the introduction, and let $J_{F}$ be the Jacobian of $\mathcal{X}_{F}$.

(a) We have

$$
\operatorname{ord}_{s=1} \zeta\left(H^{1}, s\right)=\operatorname{rank}_{\mathbb{Z}} \operatorname{Pic}^{0}(\mathcal{X})
$$

if and only if

$$
\operatorname{ord}_{s=1} L\left(J_{F}, s\right)=\operatorname{rank}_{\mathbb{Z}} J_{F}(F) .
$$

(b) In the notation of Section 4, the following statements are equivalent:

$$
\begin{aligned}
\lambda_{\infty}\left(\zeta^{*}\left(H^{1}, 1\right) \cdot \mathbb{Z}\right) & =\operatorname{det}_{\mathbb{Z}} R \Gamma_{W, c}\left(S, P^{0}\right) \otimes_{\mathbb{Z}} \operatorname{det}_{\mathbb{Z}}^{-1} H^{1}\left(\mathcal{X}, \mathcal{O}_{\mathcal{X}}\right) \\
\zeta^{*}\left(H^{1}, 1\right) & =\frac{\operatorname{vol}\left(H_{\mathrm{ar}, c}^{0}\left(S, P^{0} \otimes \tilde{\mathbb{R}} / \mathbb{Z}\right)\right)}{\operatorname{vol}\left(H_{\mathrm{ar}, c}^{1}\left(S, P^{0} \otimes \tilde{\mathbb{R}} / \mathbb{Z}\right)\right)} ; \\
\zeta^{*}\left(H^{1}, 1\right) & =\frac{\# \operatorname{Br}(\overline{\mathcal{X}}) \cdot \delta^{2} \cdot \Omega(\mathcal{X}) \cdot R(\mathcal{X})}{\left(\#\left(\operatorname{Pic}^{0}(\mathcal{X})_{\text {tor }} / \operatorname{Pic}\left(\mathcal{O}_{F}\right)\right)\right)^{2}} \cdot \prod_{v \text { real }} \frac{\# \Phi_{v}}{\delta_{v}^{\prime} \delta_{v}} .
\end{aligned}
$$

All these statements are equivalent to

$$
L^{*}\left(J_{F}, 1\right)=\frac{\# \amalg\left(J_{F}\right) \cdot \Omega(\mathcal{J}) \cdot R\left(J_{F}(F)\right)}{\left(\# J_{F}(F)_{t o r}\right)^{2}} \cdot \prod_{v} \# \Phi_{v} .
$$

Here $R\left(J_{F}(F)\right)$ is the regulator of the Néron-Tate height pairing on $J_{F}(F)$ and $\Omega(\mathcal{J})$ (resp. $\Phi_{v}$ for $\left.v \nmid \infty\right)$ was defined in Lemma 18 (resp., Lemma 17).

For the statement of the Birch and Swinnerton-Dyer conjecture for abelian varieties over number fields, we refer to seminar notes of B. Conrad [5]. Our formula (91) is equivalent to $\left[5, \mathrm{~L} 1\right.$, Conjecture 1.4.2], since the period term $\Omega_{J_{F}}$ there agrees with our

$$
\begin{aligned}
\Omega(\mathcal{J}) \cdot \prod_{v \in \Sigma} \# \Phi_{v} & =\frac{1}{I} \cdot\left(2^{-r_{2}} \sqrt{\left|D_{F}\right|}\right)^{-g} \cdot \prod_{v \in S_{\infty}} \# \Phi_{v} \cdot \mu_{v}\left(J_{F}\left(F_{v}\right)^{0}\right) \cdot \prod_{v \in \Sigma_{f}} \# \Phi_{v} \\
& =\frac{1}{I} \cdot\left(\sqrt{\left|D_{F}\right|}\right)^{-g} \cdot \prod_{v \in S_{\infty}} \tilde{\mu}_{v}\left(J_{F}\left(F_{v}\right)\right) \cdot \prod_{v \in \Sigma_{f}} \# \Phi_{v}
\end{aligned}
$$


in view of $\left[5\right.$, L3, Example 6.4] (where $\tilde{\mu}_{v}:=2 \mu_{v}$ at complex places $v$ ). For $F=\mathbb{Q}$ our statement (91) can also be found in [18, Conjecture F.4.1.6]. The original statement without explicit statement of the period term is due to Tate [35, Conjecture B].

Proof. Taking cohomology over $\bar{S}$ of the top row in diagram (71) gives an exact sequence

$$
0 \rightarrow \bigoplus_{v \in \Sigma_{f}} E_{v}^{G_{\kappa(v)}} \rightarrow \operatorname{Pic}^{0}(\mathcal{X}) / \operatorname{Pic}\left(\mathcal{O}_{F}\right) \rightarrow J_{F}(F) \rightarrow A \rightarrow 0
$$

where $A$ is a finite abelian group of cardinality $\alpha$ defined in definition (69). Since the $\mathbb{Z}$-rank of $E_{v}^{G_{\kappa(v)}}$ is $\# C_{v}-1$ by sequence (73), Theorem 6.1 (a) is then clear from equation (83).

The equivalence of the first three equations in Theorem 6.1(b) was shown in Section 4. We show directly that the third equation is equivalent to equation (91) using the previous lemmas of this section. Lemma 15 applied to sequence (92) gives

$$
\begin{aligned}
\prod_{v \in \Sigma_{f}} \Delta\left(E_{v}^{G_{\kappa(v)}}\right) \Delta\left(J_{F}(F)\right) & =\Delta\left(\operatorname{Pic}^{0}(\mathcal{X}) / \operatorname{Pic}\left(\mathcal{O}_{F}\right)\right) \Delta(A) \\
& =\frac{\Delta\left(\operatorname{Pic}^{0}(\mathcal{X}) / \operatorname{Pic}\left(\mathcal{O}_{F}\right)\right)}{\alpha^{2}}
\end{aligned}
$$

Applying Proposition 5.1, equation (93) and Lemma 17 to equation (6), we find

$$
\begin{aligned}
\zeta^{*}\left(H^{1}, 1\right) & =\frac{\# \operatorname{Br}(\overline{\mathcal{X}}) \cdot \delta^{2} \cdot \Omega(\mathcal{X}) \cdot R(\mathcal{X})}{\left(\#\left(\operatorname{Pic}^{0}(\mathcal{X})_{t o r} / \operatorname{Pic}\left(\mathcal{O}_{F}\right)\right)\right)^{2}} \cdot \prod_{v \text { real }} \frac{\# \Phi_{v}}{\delta_{v}^{\prime} \delta_{v}} \\
& =\frac{\prod_{v} \delta_{v}^{\prime} \delta_{v}}{\alpha^{2}} \# \amalg\left(J_{F}\right) \cdot \Delta\left(\operatorname{Pic}^{0}(\mathcal{X}) / \operatorname{Pic}\left(\mathcal{O}_{F}\right)\right) \cdot \Omega(\mathcal{X}) \cdot \prod_{v \text { real }} \frac{\# \Phi_{v}}{\delta_{v}^{\prime} \delta_{v}} \\
& =\prod_{v \in \Sigma_{f}}\left(\frac{\delta_{v}^{\prime} \delta_{v}}{\# \Phi_{v}} \Delta\left(E_{v}^{G_{\kappa(v)}}\right)\right) \cdot \Delta\left(J_{F}(F)\right) \cdot \# \amalg\left(J_{F}\right) \cdot \Omega(\mathcal{X}) \cdot \prod_{v \in \Sigma} \# \Phi_{v} \\
& =\prod_{v \in \Sigma_{f}}\left(\log N v^{\left|C_{v}\right|-1} \prod_{i \in C_{v}} r_{v, i}\right) \cdot \Delta\left(J_{F}(F)\right) \cdot \# \amalg\left(J_{F}\right) \cdot \Omega(\mathcal{X}) \cdot \prod_{v \in \Sigma} \# \Phi_{v},
\end{aligned}
$$

and equation (84) and Lemma 18 show that this identity is equivalent to

$$
\begin{aligned}
L^{*}\left(J_{F}, 1\right) & =\Delta\left(J_{F}(F)\right) \cdot \# \amalg\left(J_{F}\right) \cdot \Omega(\mathcal{X}) \cdot \prod_{v \in \Sigma} \# \Phi_{v} \\
& =\frac{R\left(J_{F}(F)\right)}{\left(\# J_{F}(F)_{t o r}\right)^{2}} \cdot \# \amalg\left(J_{F}\right) \cdot \Omega(\mathcal{J}) \cdot \prod_{v \in \Sigma} \# \Phi_{v},
\end{aligned}
$$

which is equation (91). Here we also use the fact that the Arakelov intersection pairing induces the negative of the Néron-Tate height pairing on $J_{F}(F)$ by [19, Theorem 3.1].

Remark 4. If $\mathcal{X}_{F}$ has genus 0 - that is, is a twisted form of $\mathbb{P}_{F}^{1}-$ then $L\left(J_{F}, s\right)=1$ and the Birch and Swinnerton-Dyer conjecture is trivially true. So is our conjecture for 
$\zeta\left(H^{1}, s\right)$. One has an isomorphism

$$
\operatorname{Pic}^{0}(\mathcal{X}) / \operatorname{Pic}\left(\mathcal{O}_{F}\right) \cong \bigoplus_{v \in \Sigma_{f}} E_{v}^{G_{\kappa(v)}}
$$

and the formula

$$
\zeta^{*}\left(H^{1}, 1\right)=\# \operatorname{Br}(\overline{\mathcal{X}}) \cdot \delta^{2} \cdot \prod_{v \in \Sigma_{f}} \Delta\left(E_{v}^{G_{\kappa(v)}}\right) \cdot \prod_{v \text { real }} \frac{1}{\delta_{v}^{\prime} \delta_{v}}
$$

can be verified directly from equation (84), Lemma 17 and Proposition 5.1. Since a genus 0 curve has a point in a quadratic extension, we have $\delta, \delta_{v} \in\{1,2\}$ and we also have $\delta_{v}^{\prime}=1$ for all $v$ by [22]. The number of places where $\delta_{v}=2$ is even, since the twisted forms of $\mathbb{P}_{F}^{1}$ are parameterised by $\operatorname{Br}(F)[2]$. So we verify directly that

$$
\# \operatorname{Br}(\overline{\mathcal{X}})=\delta^{-2} \prod_{v} \delta_{v}
$$

is a square.

Remark 5. Let

$$
\bar{f}: \overline{\mathcal{X}} \rightarrow \bar{S}
$$

be a flat morphism from a smooth projective surface $\overline{\mathcal{X}}$ to a smooth projective connected curve $\bar{S} \stackrel{\pi}{\rightarrow} \operatorname{Spec}(k)$ over a finite field $k$, and assume $\bar{f}$ has geometrically connected fibres. By [1, Remark 5.4.9],

$$
K:=R \bar{f}_{*} \mathbb{Q}_{l}
$$

is a pure complex in the derived category of $l$-adic sheaves on $\bar{S}$, and by $[1$, Theorems 5.4 .5 and 5.4.10], we have

$$
\begin{aligned}
K & \cong{ }^{p} \mathcal{H}^{1}(K)[-1] \oplus{ }^{p} \mathcal{H}^{2}(K)[-2] \oplus{ }^{p} \mathcal{H}^{3}(K)[-3] \\
& \cong \mathbb{Q}_{l}[0] \oplus^{p} \mathcal{H}^{2}(K)[-2] \oplus \mathbb{Q}_{l}(-1)[-2],
\end{aligned}
$$

where ${ }^{p} \mathcal{H}^{i}$ refers to the perverse $t$-structure (which agrees with the ordinary $t$-structure over $\operatorname{Spec}(k))$. Assume in addition that $\bar{f}$ has large monodromy in the sense that $R^{0} \pi_{*} R^{1} \bar{f}_{*} \mathbb{Q}_{l}=0$. With notation as in the proof of Lemma 16, we have

$$
{ }^{p} \mathcal{H}^{2}(K)=\left(R^{1} \bar{f}_{*} \mathbb{Q}_{l}\right)[1] \oplus E \otimes \mathbb{Q}_{l}(-1)[0],
$$

and hence our assumption implies $R^{-1} \pi_{*}^{p} \mathcal{H}^{2}(K)=0$, which together with [1, Theorem 5.4.10] gives

$$
R^{i} \pi_{*}^{p} \mathcal{H}^{2}(K)=0, \quad i \neq 0
$$

We find

$$
\begin{aligned}
R^{2}(\pi \circ \bar{f})_{*} \mathbb{Q}_{l} \cong R^{2} \pi_{*}(K) & \cong R^{2} \pi_{*} \mathbb{Q}_{l} \oplus R^{0} \pi_{*}^{p} \mathcal{H}^{2}(K) \oplus R^{0} \pi_{*} \mathbb{Q}_{l}(-1) \\
& \cong \mathbb{Q}_{l}(-1) \oplus R \pi_{*}^{p} \mathcal{H}^{2}(K) \oplus \mathbb{Q}_{l}(-1)
\end{aligned}
$$


The zeta function of the $l$-adic sheaf $R^{2}(\pi \circ \bar{f})_{*} \mathbb{Q}_{l}$ was referred to as $\zeta\left(H_{a b s}^{2}, s\right)$ in the introduction, and the zeta function of the $l$-adic complex $R \pi_{*}^{p} \mathcal{H}^{2}(K)$ is the function $\zeta\left(H^{1}, s\right)$ discussed in this paper, albeit over the base $\bar{S}$ rather than $S$. By formula (94) we have

$$
\zeta\left(H_{a b s}^{2}, s\right)=\zeta\left(H^{1}, s\right)\left(1-q^{1-s}\right)^{2},
$$

where $q=\# k$. It seems likely that the proof in [11] of the equivalence of the conjecture of Artin and Tate for $\zeta^{*}\left(H_{a b s}^{2}, 1\right)$ [35] with the Birch and Swinnerton-Dyer conjecture for the Jacobian of the generic fibre of $\bar{f}$ can be somewhat simplified using our approach to $\zeta^{*}\left(H^{1}, 1\right)$ via the complex $P^{0}$.

\section{Acknowledgements}

We would like to thank the referee for many suggestions which have considerably improved the exposition of this paper. The authors declare no conflicts of interest.

\section{References}

[1] A. Beilinson, J. Bernstein and P. Deligne, Faisceaux pervers: Analyse et topologie sur les éspaces singuliers, Astérisque, 100 (Société Mathématique de France, Paris, 1982).

[2] S. Bloch, De Rham cohomology and conductors of curves, Duke Math. J. 54(2) (1987), 295-308.

[3] S. Bosch And Q. Liu, Rational points of the group of components of a Neron model, Manuscripta Math. 98(3) (1999), 275-293.

[4] J. L. Colliot-Thélène and S. SAito, Zéro-cycles sur les variétés p-adiques et groupe de Brauer, Int. Math. Res. Not. IMRN 4 (1996), 151-160.

[5] B. Conrad, 'Seminar notes on the Birch and Swinnerton-Dyer conjecture', 2015, http://virtualmath1.stanford.edu/conrad/BSDseminar/.

[6] M. Flach And B. Morin, Weil-étale cohomology and Zeta-values of proper regular arithmetic schemes, Doc. Math. 23 (2018), 1425-1560.

[7] M. Flach and B. Morin, Deninger's conjectures and Weil-Arakelov cohomology, Münster J. Math., 13(2) (2020), 519-540.

[8] K. Fujiwara, A proof of the absolute purity conjecture (after Gabber), in Algebraic Geometry 2000, Azumino (Hotaka), Advanced Studies in Pure Mathematics, 36 pp. 153183 (Mathematical Society of Japan, Tokyo, 2002).

[9] T. Geisser, Arithmetic cohomology over finite fields and special values of $\zeta$-functions, Duke Math. Jour. 133(1) (2006), 27-57.

[10] T. Geisser, Comparing the Brauer to the Tate-Shafarevich group, J. Inst. Math. Jussieu, 19(3) (2020), 965-970.

[11] W. J. Gordon, Linking the conjectures of Artin-Tate and Birch-Swinnerton-Dyer, Compos. Math. 38(2) (1979), 163-199.

[12] B. H. Gross and J. Harris, Real algebraic curves, Ann. Sci. Éc. Norm. Supér. (4) 14 (1981), 157-182.

[13] A. Grothendieck, Techniques de descente et théorèmes d'existence en géométrie algébrique VI, in Les schémas de Picard: propriétés générales, Séminaire Bourbaki, 236, pp. 221-243 (Société Mathématique de France, Paris, 1962). 
[14] A. Grothendieck, Le groupe de Brauer III: Dix exposés sur la cohomologie des schémas (North Holland, Amsterdam, 1968).

[15] A. Grothendieck, Revétements étales et groupe fondamental (SGA 1), Lecture Notes in Mathematics, 224 (Springer, Berlin-Heidelberg, 1971).

[16] A. Grothendieck, M. Artin and J. L. Verdier, Theorie des Topos et Cohomologie Etale des Schemas (SGA 4), Lecture Notes in Mathematics 269, 270, 271 (Springer, BerlinHeidelberg, 1972).

[17] R. Hartshorne, Algebraic Geometry, Graduate Texts in Mathematics, 52 (Springer, New-York, 1977).

[18] S. Hindry and J. Silverman, Diophantine Geometry: An Introduction, Graduate Text in Mathematics, 201 (Springer, New-York, 2000).

[19] P. Hriluac, Heights and Arakelov's intersection theory, Amer. J. Math. 107(1) (1985), 23-38.

[20] L. Illusie, Complexe cotangent et déformations. I, Lecture Notes in Mathematics, 239 (Springer, Berlin-Heidelberg, 1971).

[21] Y. Li, Y. LiU And Y. Tian, 'On the Birch and Swinnerton-Dyer conjecture for CM elliptic curves over $\mathbb{Q}^{\prime}$, Preprint, 2016, https://arxiv.org/abs/1605.01481v1.

[22] S. Lichtenbaum, Duality theorems for curves over p-adic fields, Invent. Math. 7 (1969), $120-136$.

[23] Q. Liu, D. Lorenzini and M. Raynaud, Néron models, Lie algebras, and reduction of curves of genus one, Invent. Math. 157 (2004), 455-518.

[24] Q. Liu, D. Lorenzini and M. Raynaud, Corrigendum to Néron models, Lie algebras, and reduction of curves of genus one and The Brauer group of a surface, Invent. Math. 214 (2018), 593-604.

[25] J. LuRIE, Higher Algebra, 2017, http://www.math.ias.edu/lurie/papers/HA.pdf.

[26] J.S. Milne, Étale Cohomology, Princeton Mathematical Series, 17 (Princeton University Press, Princeton N.J., 1980).

[27] J.S. Milne, Arithmetic Duality Theorems, Perspectives in Mathematics, 1 (Academic Press, Boston, 1986).

[28] B. Poonen and M. Stoll, The Cassels-Tate pairing on polarized abelian varieties, Annals of Math. 150 (1999), 1109-1149.

[29] M. Raynaud, Spécialisation du foncteur du Picard, Publ. Math. Inst. Hautes Études Sci. 38 (1970), 27-76.

[30] S. Saito, Arithmetic theory of arithmetic surfaces, Ann. Math. 129 (1989), 547-589.

[31] K. Sato, p-adic étale twists and arithmetic duality, Ann. Sci. Éc. Norm. Supér. (4) 40(4) (2007), 519-588.

[32] C. Soulé, D. Abramovich, J.-F. Burnol and J. Kramer, Lectures on Arakelov Geometry, (Cambridge University Press, Cambridge U.K., 1992).

[33] M. Spiess, Artin-Verdier duality for arithmetic surfaces, Math. Ann. 305 (1996), 705-792.

[34] The Stacks Project Authors, The Stacks Project, 2019, http://stacks.math. columbia.edu.

[35] J. TAte, On the conjecture of Birch and Swinnerton-Dyer and a geometric analogue, Sém. Bourbaki, 306, pp. 1-26 (Société Mathématique de France, Paris, 1966).

[36] V. Voevodsky, Homology of schemes, Selecta Math. (N. S.) 2(1) (1996), 111-153.

[37] X. WAN, 'Iwasawa main conjecture for supersingular elliptic curves and BSD conjecture', Preprint, 2019, https://arXiv.org/abs/1411.6352v6.

[38] C. Zhong, Comparison of dualizing complexes, J. Reine Angew. Math. 695 (2014), 1-39. 